\title{
Potential for Photoacoustic Imaging of Neonatal Brain
}

Pantea Tavakolian, The University of Western Ontario

Supervisor: Dr. Jeffrey Carson, The University of Western Ontario

A thesis submitted in partial fulfillment of the requirements for the Master of Science degree in Medical Biophysics

(C) Pantea Tavakolian 2014

Follow this and additional works at: https://ir.lib.uwo.ca/etd

Part of the Bioimaging and Biomedical Optics Commons, and the Biomedical Devices and Instrumentation Commons

\section{Recommended Citation}

Tavakolian, Pantea, "Potential for Photoacoustic Imaging of Neonatal Brain" (2014). Electronic Thesis and Dissertation Repository. 2372.

https://ir.lib.uwo.ca/etd/2372

This Dissertation/Thesis is brought to you for free and open access by Scholarship@Western. It has been accepted for inclusion in Electronic Thesis and Dissertation Repository by an authorized administrator of Scholarship@Western. For more information, please contact wlswadmin@uwo.ca. 
POTENTIAL FOR PHOTOACOUSTIC IMAGING OF NEONATAL BRAIN

(Thesis format: Integrated Article)

by

Pantea Tavakolian

Graduate Program in Medical Biophysics

\begin{abstract}
A thesis submitted in partial fulfillment of the requirements for the degree of Master of Science
\end{abstract}

The School of Graduate and Postdoctoral Studies

The University of Western Ontario

London, Ontario, Canada

(C) Pantea Tavakolian 2014 


\section{Abstract}

Photoacoustic imaging is a hybrid imaging technique that combines many of the merits of both optical and ultrasound imaging. Photoacoustic imaging (PAI) has been hypothesized as a technique for imaging neonatal brain. However, PAI of the brain is more challenging than traditional methods (e.g. near infrared spectroscopy) due to the presence of the skull layer. To evaluate the potential limits the skull places on 3D PAI of the neonatal brain, we constructed a neonatal skull phantom $(\sim 1.52-\mathrm{mm}$ thick) with a mixture of epoxy and titanium dioxide powder that provided acoustic insertion loss $(1-5 \mathrm{MHz})$ similar to human infant skull bone. The phantom was molded into a realistic infant skull shape by means of a CNCmachined mold that was based upon a 3D CAD model. Then, propagation of photoacoustic (PA) signals through the skull phantom was examined. A photoacoustic point source was raster-scanned within the imaging cavity of a 128-channel PAI system to capture the imaging operator with and without the intervening skull phantom layer. Then, effects of the skull phantom on PA signals and consequently on PA images was evaluated in detail. We captured 3D photoacoustic images of tubes filled with indocyanine green (ICG). The system was capable of reconstructing an image of a tube filled with $50 \mu \mathrm{M}$ ICG in presence of the skull. Image processing method was developed to correct photoacoustic images from the effects of the skull. The method was tested on an image of an object captured through the skull, which demonstrated that the effects of the skull on PA images are predictable and modifiable.

\section{Keywords}

3D photoacoustic imaging (PAI), photoacoustic tomography, optoacoustic tomography, skull mimicking phantom, ultrasound attenuation, periventricular leukomalacia. 


\section{Co-Authorship Statement}

This section describes the contribution from various authors for the work completed in Chapters $2 \& 3$.

Chapter 2: P. Tavakolian, I. Kosik, Lawrence, K. S., J.J.L. Carson. "Investigation of 3D photo acoustic neonatal brain imaging using a skull-mimicking phantom”, Journal of Biomedical Optics.

Ivan Kosik aided in the design the photoacoustic imaging system. Dr. Carson oversaw project design, and analysis of results. He designed the photoacoustic imaging system. He edited the manuscript, and provided general project supervision. Dr. Lawrence contributed in editing the manuscript. I aided in the design and fabrication of the skull molds, construction of the skull phantoms. I performed all experiments, analyzed and interpreted the results, and wrote the manuscript. The manuscript is being prepared for submission to the Journal of Biomedical Optics.

Chapter 3: P. Tavakolian, Lawrence, K. S., J.J.L. Carson. "Investigation of the effects of a skull on 3D photoacoustic neonatal brain imaging", Journal of Biomedical Optics.

Dr. Carson oversaw project design, and analysis of results. He designed the photoacoustic imaging system. He edited the manuscript, and provided general project supervision. Dr. Lawrence contributed in editing the manuscript. I performed all experiments, analyzed and interpreted the results, and wrote the manuscript. This chapter will change to a format of a manuscript for submission to the Journal of Biomedical Optics. 


\section{Acknowledgments}

I would like to express my strongest appreciation to my supervisor, Prof. Jeff Carson, who continually and convincingly conveyed a spirit of adventure in regard to research. He supported me throughout my Masters with his everlasting patience and knowledge. Without his understanding, patience, guidance, and persistent help adaption to new environment, and this dissertation would not have been possible. I would also like to thank my co-supervisor, Prof. Keith St. Lawrence, for his comments.

I would like to thank many teachers, colleagues, and friends who directly or indirectly helped me throughout my Master, Prof. Terry Thompson, Astrid Champson-Reig, Avery Raess, Fartash Vasefi, Morteza Heydari Araghi, Ivan Kosik, Mohammadreza Najiminaini, Philip Wong, Eldon Ng, Rhiannon Todd, and many fellow students.

Lastly, I wish to thank my parents, Reza and Shamsi, who raised me, supported me, taught me, and loved me, and my brothers, Kouhyar and Kamyar, who are my whole world, and have always been there for me. I dedicate this thesis to them. 


\section{Table of Contents}

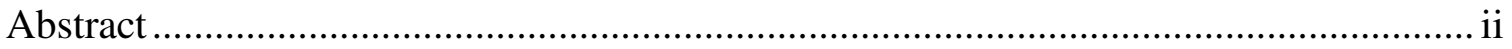

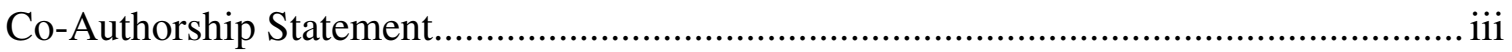

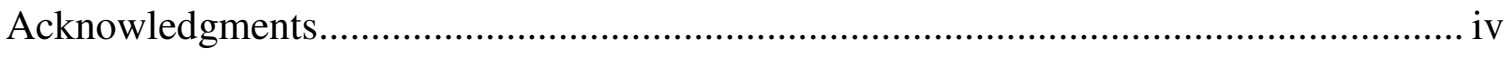

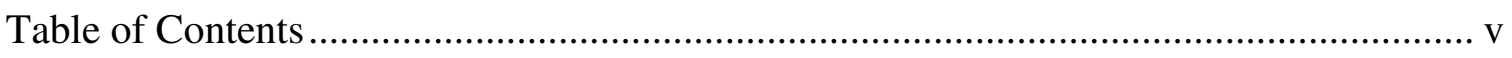

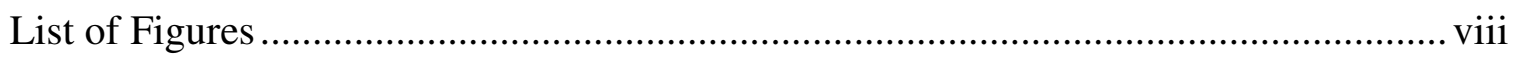

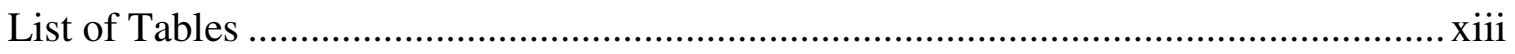

List of Abbreviations and Symbols................................................................ xiv

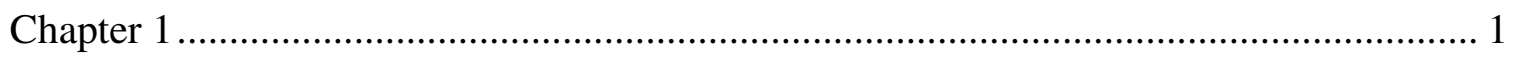

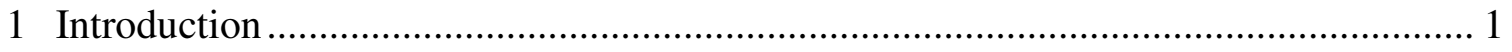

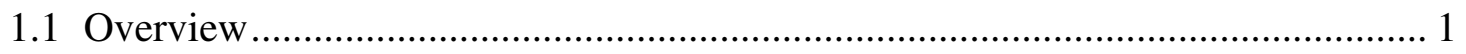

1.2 Fundamentals of Photoacoustic imaging .................................................... 1

1.2.1 Photoacoustic signal generation......................................................... 3

1.2.2 The photoacoustic wave................................................................... 4

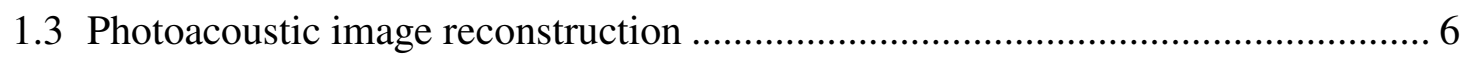

1.3.1 Pseudoinverse of the imaging operator ............................................ 7

1.3.2 Regularization of the imaging operator ............................................. 8

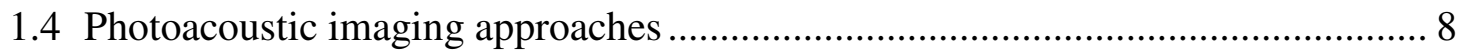

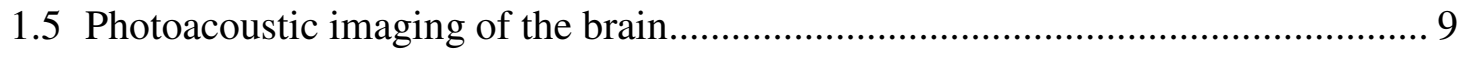

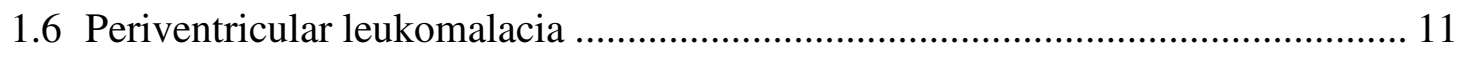

1.7 Acoustic properties of the neonatal skull bone ............................................ 15

1.8 Light attenuation through the neonatal skull bone......................................... 16

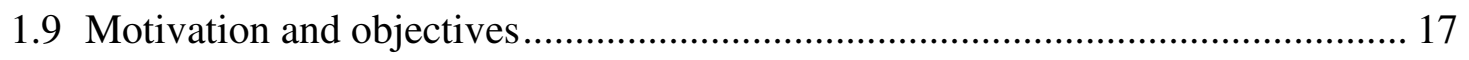

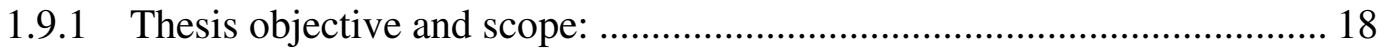

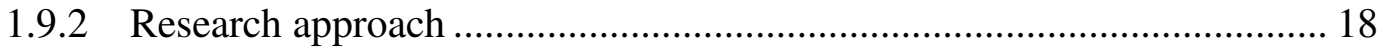


2 Investigation of 3D photo acoustic neonatal brain imaging using a skull-mimicking

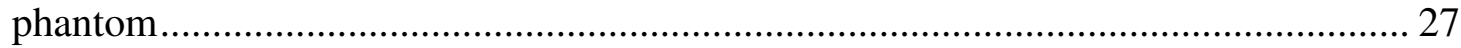

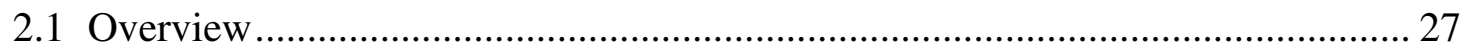

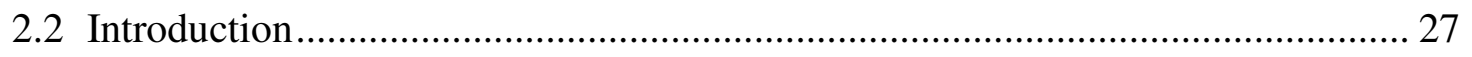

2.2.1 Background and motivation .......................................................... 27

2.2.2 Objective and approach........................................................... 29

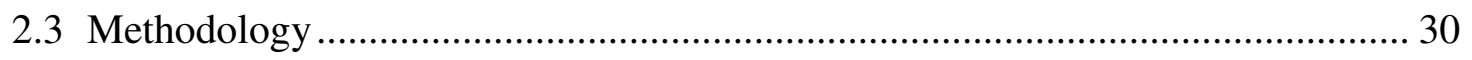

2.3.1 System setup ........................................................................ 30

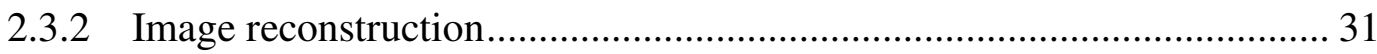

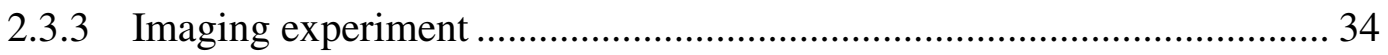

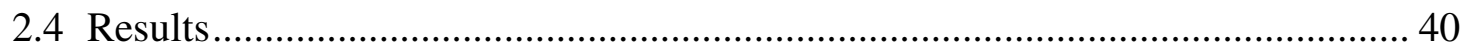

2.4.1 Skull Phantom.......................................................................... 40

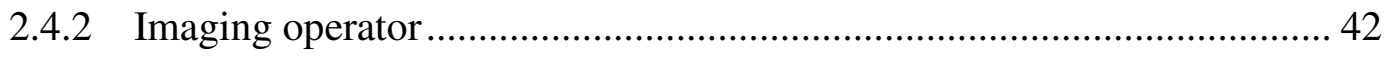

2.4.3 Photoacoustic imaging of different objects..................................... 46

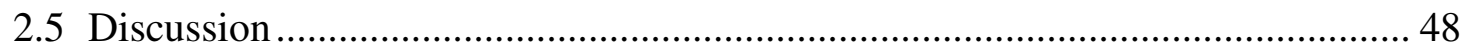

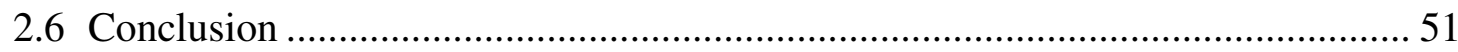

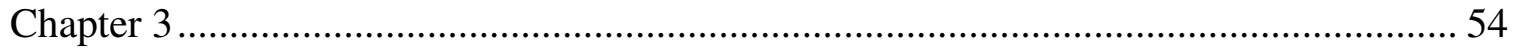

3 3D photoacoustic imaging through the skull phantom with phase and amplitude

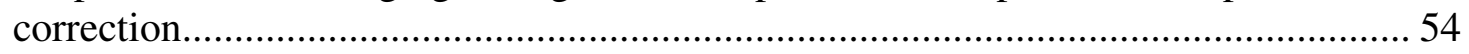

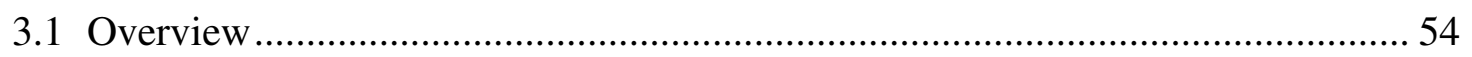

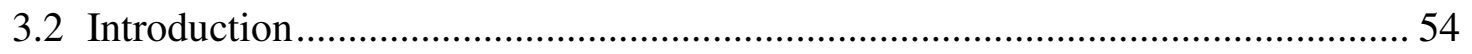

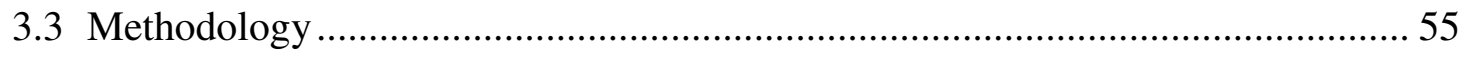

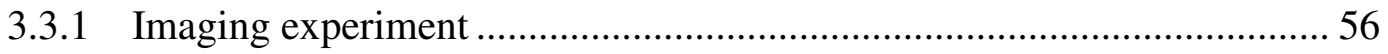

3.3.2 Photoacoustic signal enhancement method …..................................... 58

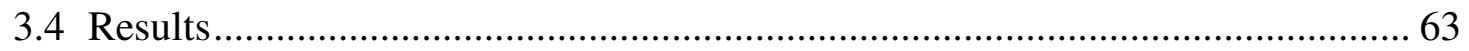

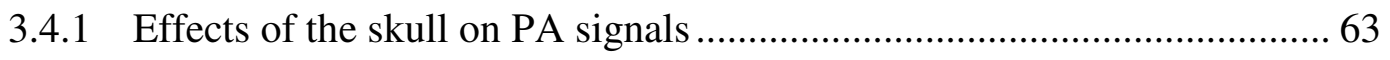




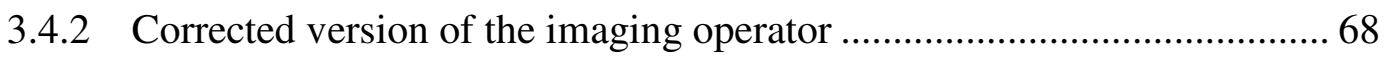

3.4.3 Image enhancement ......................................................................... 70

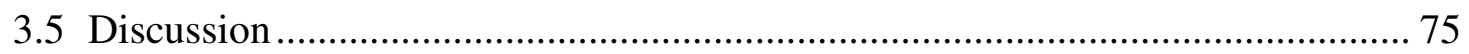

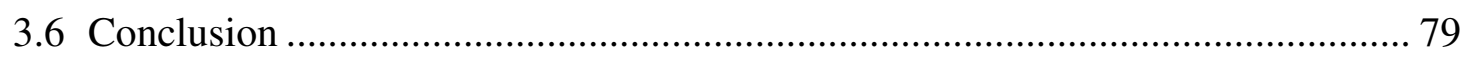

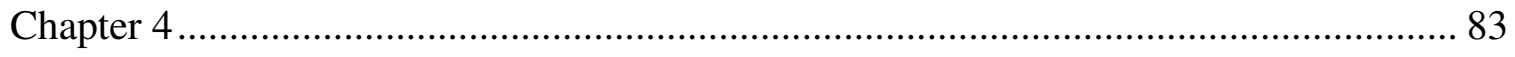

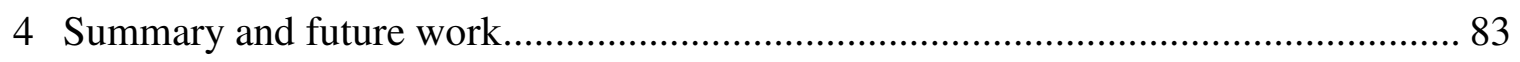

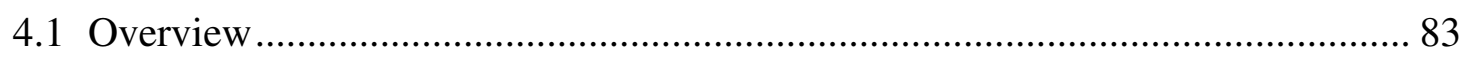

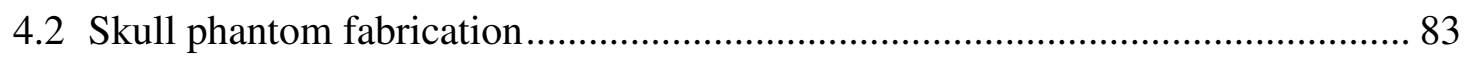

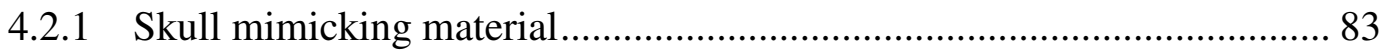

4.2.2 Skull mold fabrication....................................................................... 84

4.3 Effects of the skull layer on photoacoustic signals .............................................. 84

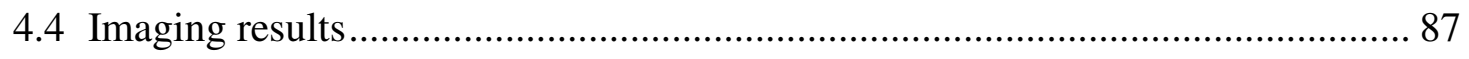

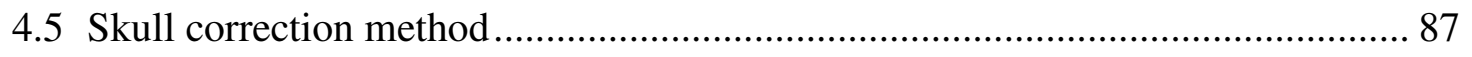

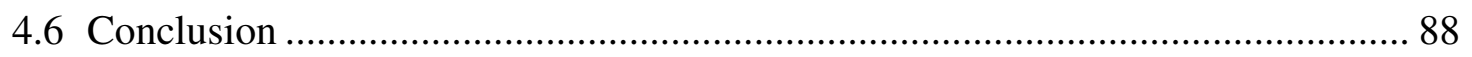

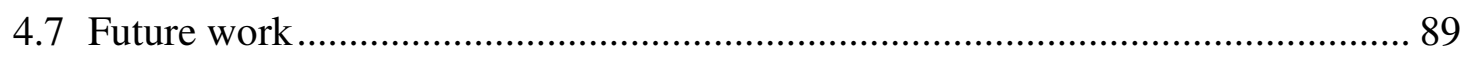

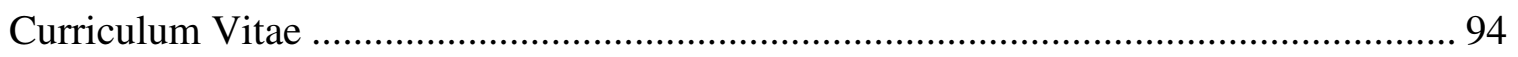




\section{List of Figures}

Figure 1.1. Photoacoustic signal profile in the time domain that contains the maximum amplitude of $\mathrm{P} 0 R 0 / r$, and width equal to twice the quotient of $\mathrm{R} 0 / v$. The spherical source has a radius of $\mathrm{R} 0$, and $\mathrm{V}$ is the speed of sound in the propagating medium. 5

Figure 1.2. Coronal section of cerebrum. In one hemisphere focal and diffuse components of PVL are shown and in the other hemisphere vascular supply to the cerebral white matter is

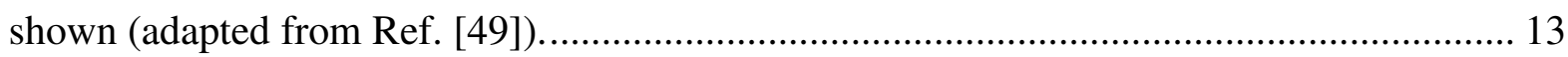

Figure 1.3. Cystic periventricular leukomalacia. (a) Sagittal and (b) coronal ultrasound images show cystic PVL (arrows). These images were acquired 7 weeks after birth of a preterm infant with 31 weeks gestational age (adapted from Ref. [50]). 14

Figure 1.4. Ultrasound insertion loss of an infant skull as a function of frequency (adapted from Ref. [32]). 16

Figure 1.5. Light transmittance through the infant skull as a function wavelength. (adapted from Ref. [32]) 17

Figure 2.1. (a) Photoacoustic imaging system which includes a spherical imaging cavity consisting of 127 ultrasound transducers, a DAQ system, two lasers, a delay generator, two computers, a robot and its controller, and a photoacoustic point source attached to the robot; (b) Blue curve is the maximum amplitude of the PA signals detected at the monitoring transducer at all grid points for the calibration scan performed in the absence of the skull; green curve is the time of arrival of these PA signals; red curves display the mean value and standard deviation of the blue and green curves. (c) The same parameters described in (b), this time for the calibration scan captured through the skull.

Figure 2.2. (a) Skull mold, (b) a 3D CAD model of the skull phantom, (c) a skull phantom which has a transcranial thickness of $1.52 \mathrm{~mm}$, a length of $79.3 \mathrm{~mm}$, width of $63 \mathrm{~mm}$ (not including flanges), and a height of $31.5 \mathrm{~mm}$, and (d) a 3D CAD model of the transducer array with the skull in place during the calibration scan; tip of the fiber optic that is attached to the robot is the point source; the green lines represent the central lines of the scan at the middle $x y$ plane and the red and blue lines represent the grid locations at the opposite side of the $x$ 
axis and along the $x$ axis, close to the front and back edges of the skull; (e) a 3D CAD model of the positions of transducers 1,2, and 3 in relation to the scan volume (red cuboid) and also location of three of the four fibers that illuminates center of the transducer array, (f) a 3D CAD model of the position of the two tubes (green cylinder) in the transducer array. 38

Figure 2.3. Acoustic insertion loss of different samples prepared from mixtures of epoxy and titanium dioxide powder. (a) Acoustic insertion loss of the samples composed of 50\% epoxy and $50 \%$ titanium dioxide powder with different thicknesses compared to acoustic insertion loss of the skull (adapted from Ref $[28,43]$ ), (b) Acoustic insertion loss of samples with the same thickness $(\sim 1.4 \mathrm{~mm})$ and different proportions of titanium dioxide powder compared to acoustic insertion loss of neonatal skull.

Figure 2.4. (a) three representative subsets of the imaging operator measured without the skull phantom at three different locations (the three transducers are shown in Figure 2.2 (e)): four scan lines being $\sim 9 \mathrm{~mm}$ from the center of the volume on the opposite side of the $x$ axis (four red lines in Figure 2.2 (d)); four central scan lines (four green lines in Figure 2.2 (d)); four scan lines being $\sim 9 \mathrm{~mm}$ from the center of the imaging along the $\mathrm{x}$ axis (four blue lines in Figure 2.2 (d)); (b) three subsets of the imaging operator measured with the skull phantom, at the same locations and the same transducers as (a). Each row of each subset shows data from transducer one, two, and three (Figure 2.2 (e)) at 160 grid locations. Only 250 time points are displayed for each transducer signal due to data preprocessing procedures that involved subset selection and down-sampling of the data. 44

Figure 2.5. Photoacoustic images of one tube, and two tubes, filled with different concentrations of ICG $(500 \mu \mathrm{M}, 250 \mu \mathrm{M}, 100 \mu \mathrm{M}, 50$, and $1.25 \mu \mathrm{M})$, and captured in the absence and the presence of the skull phantom. The threshold applied for images of the tubes with $1.25 \mu \mathrm{M}$ ICG was half of the threshold applied for other images.

Figure 3.1. Photoacoustic signal propagation through a 1.4-mm thick skull phantom composed of 50\% epoxy and 50\% titanium dioxide powder. The red curve represents the PA signal without the skull in place and the blue curve represents the PA signal on the same transducer after passing through the skull layer. The PA point source was located near the center of the array. 59 
Figure 3.2. (a) Red curve: example of a PA signal captured in the absence of the skull at a transducer and one grid location. Peak of this signal is located at A in time trace. (a) Blue curve: the PA signal captured through the skull at the same transducer and the same voxel as red curve. Peak of this PA signal is located at B in time trace. (b) This waveform displays the result of cross-correlation of the two PA signal displayed in panel (a). The peak of this signal occurs at $\mathrm{C}(\mathrm{C}=250-(\mathrm{A}-\mathrm{B}))$ in time trace. 61

Figure 3.3. Normalized cross-correlation between PA signals captured through the skull and in the absence of the skull at all 127 transducers. In each panel, the value shows the normalized cross-correlation at each grid point of the imaging operator within a plane. Three $x y$ planes $(z=0 \mathrm{~mm}, z=5 \mathrm{~mm}, z=10 \mathrm{~mm})$ are displayed in the figure; (a) the first plane (closest to the surface of the skull), (b) the middle plane (central plane), and (c) the last plane (furthest from the surface of the skull). These axes are from 0 to 40 steps; therefore, based on the step size of the scan which is $0.5 \mathrm{~mm}$, each panel covers a square of $20 \mathrm{~mm} \times 20 \mathrm{~mm}$. . 64

Figure 3.4. (a) PAI cavity showing positions of transducers 1, 2, and 3. (b) Scaling maps. Each value in each panel represents the amplitude attenuation of the PA signal due to the presence of the skull phantom at a specific voxel (grid location). In each panel the $x$ and $y$ axes are equal to the $x$ and $y$ axes shown in (a). The axes increase in index from 0 to 40. As mentioned above, each panel covers a square of $20 \mathrm{~mm} \times 20 \mathrm{~mm}$, based on the step size of the scan which was $0.5 \mathrm{~mm}$. 66

Figure 3.5. Delay maps. Each value in each panel shows the differences in time of arrival of the PA signal with and without the skull at a specific voxel (grid location). In each panel the $x$ and $y$ axes are equal to the $x$ and $y$ axes shown in Figure 3.4 (a). The axes are increasing from 0 to 40 steps. Each panel covers a square of $20 \mathrm{~mm} \times 20 \mathrm{~mm}$. Due to the PA sampling rate which was set to $40 \mathrm{MHz}$, each delay time point was $25 \mathrm{~ns}$. Therefore, for all the panels, the delay values range from 0 to $1 \mu \mathrm{s}$. 67

Figure 3.6. Demonstration of the data correction method. (a) Subset of the imaging operator measured in the absence of the skull phantom and at the center of the imaging volume $(z=$ $5 \mathrm{~mm}$ ). (b) The same subset of the imaging operator measured in the presence of the skull. (c) The skull-corrected version of (a), which predicted the effects of the skull layer in terms of amplitude attenuation and shift in time of arrival. (d) Subset of the imaging operator 
measured in the absence of the skull phantom and at one edge of the imaging volume (the first $x y$ plane $(z=0 \mathrm{~mm}))$. (e) The same subset of the imaging operator as (d) measured in the presence of the skull. (f) The skull-corrected version of (d). Each row of each subset shows data from transducer one (Figure 3.4 (a)) at 160 central grid locations (4 central scan lines) of the imaging volume. Only 150 of possible 250 time points are displayed to enhance visualization.

Figure 3.7. Position of a optically-absorbing rod with a diameter of $0.4 \mathrm{~mm}$ and length of 14 $\mathrm{mm}$ in relation to the skull and the imaging cavity. (b) A PA image of the rod captured in the absence of the skull and reconstructed with the original IO. (c) A PA image of the rod captured in the presence of the skull and reconstructed with the original IO. (d) A PA image of the rod captured in the presence of the skull and reconstructed with the IO captured in the presence of the skull. (e) A PA image of the rod captured in the presence of the skull and reconstructed with the skull-corrected IO.

Figure 3.8. Averaged intensity along $20 x y$ planes of the rod (left column) and along a line normal to the rod (right column). The red lines on the panels in the left column display the location of the path used to extract the line profiles in the right column. On the panels in right column, averaged intensity profiles are displayed (blue line). On these diagrams, the width at the 12 of the line intensity profile is displayed with a black dotted line. (a) The intensity profile for the image that was captured in the absence of the skull and reconstructed with the water-only IO. (b) The intensity profile for the image that was captured in the presence of the skull and reconstructed with the water-only IO. (c) The intensity profile for the image that was captured in the presence of the skull and reconstructed with the IO captured through the skull. (d) The intensity profile for the image that was captured in the presence of the skull and reconstructed with the skull-corrected IO. 74

Figure 4.1. (a) A 3D CAD model of the skull position in relation to the transducer array, the imaging volume, and an optically-absorbing rod. (b) A 3D CAD model of the skull position, which was turned $37^{\circ}$ in the xy plane. (c) Image of the rod through the skull reconstructed with the original imaging operator. The skull was located in the same place as that where the imaging operator was captured (shown in (a)). (d) Image of the rod through the skull that was turned $37^{\circ}$ in the xy plane. The image is reconstructed with the original imaging operator. (e) 
An image of the rod reconstructed with the imaging operator that was captured through the skull. Capturing the signal of the rod, and the imaging operator, were both at $0^{\circ}$ (f) Image of the rod reconstructed with the imaging operator that was captured through the skull. To capture the data of the rod the skull was rotated $37^{\circ}$, but to capture the imaging operator the

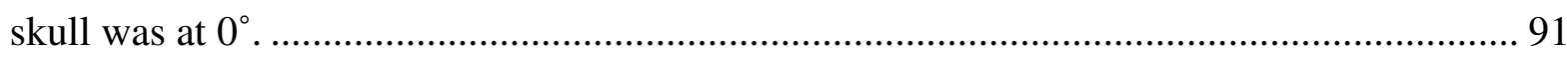




\section{List of Tables}

Table 3.1. Signal to noise ratio of the images of the rod (shown in Figure 3.7).................. 72 


\section{List of Abbreviations and Symbols}

\begin{tabular}{|c|c|}
\hline $2 \mathrm{D}$ & Two dimensional \\
\hline $3 \mathrm{D}$ & Three dimensional \\
\hline 4D & Four dimensional \\
\hline $\mathrm{cm}$ & Centimeter \\
\hline CAD & Computer-aided design \\
\hline CAM & Computer-aided-Manufacturing \\
\hline $\mathrm{CBF}$ & Cerebral blood flow \\
\hline $\mathrm{CT}$ & Computed tomography \\
\hline $\mathrm{CV}$ & Coefficient of variation \\
\hline DAQ & Data acquisition system \\
\hline $\mathrm{dB}$ & Decibel \\
\hline FWHM & Full width half maximum \\
\hline GB & Gigabyte \\
\hline $\mathrm{Hb}$ & deoxygenated-hemoglobin \\
\hline $\mathrm{HbO}_{2}$ & oxygenated-hemoglobin \\
\hline HIFU & High intensity focused ultrasound \\
\hline ICG & Indocyanine green \\
\hline J & Joule \\
\hline K & Kelvin \\
\hline
\end{tabular}




\begin{tabular}{|c|c|}
\hline $\mathrm{m}$ & Meter \\
\hline $\mathrm{MHz}$ & Megahertz \\
\hline $\mathrm{mm}$ & Millimeter \\
\hline MRI & Magnetic resonance imaging \\
\hline $\mathrm{ms}$ & Millisecond \\
\hline ns & Nanosecond \\
\hline PA & Photoacoustic \\
\hline PAI & Photoacoustic imaging \\
\hline PAT & Photoacoustic tomography \\
\hline PVL & Periventricular leukomalacia \\
\hline $\mathrm{s}$ & second \\
\hline SNR & Signal-to-noise ratio \\
\hline SVD & Singular value decomposition \\
\hline US & Ultrasound \\
\hline$\mu \mathrm{m}$ & Micrometer \\
\hline$\mu \mathrm{M}$ & Micromolar \\
\hline$\mu \mathrm{s}$ & Microsecond \\
\hline$\pi$ & $\mathrm{Pi}$ \\
\hline
\end{tabular}




\section{Chapter 1}

\section{Introduction}

\subsection{Overview}

Periventricular Leukomalacia, especially its focal component, is a common white matter brain injury in premature infants. The motivation of this work was to study the possibility of using a 3D photoacoustic imaging system for detection of such damages and consequently for neonatal brain imaging. Imaging the brain through the skull is challenging since the skull layer introduces strong image distortions. In this work, a series of simulations and experiments on a skull-mimicking phantom were performed so that the distorting effects of the skull layer could be identified and subsequently corrected for in the brain images.

This chapter introduces several relevant subjects and is organized as follows. The fundamentals of photoacoustic imaging are introduced in Section 1.2. The concepts related to photoacoustic image reconstruction are presented in Section 1.3. Different photoacoustic imaging approaches are reviewed in Section 1.4. A review of literature for

photoacoustic brain imaging is presented in Section 1.5. In Section 1.6, Background relevant to the pathogenesis of PVL is described. The details of the acoustic and optical properties of neonatal skull are introduced in Section 1.7 and 1.8 respectively. Finally, the motivations and objectives of this work are described further in Section 1.9.

\subsection{Fundamentals of Photoacoustic imaging}

The photoacoustic effect was first discovered by Alexander Graham Bell in the 1880s. After a century, in 1982, Theodore Bowen suggested the feasibility of this phenomenon 
in soft tissue imaging [1]. In 1994, Kruger demonstrated the photoacoustic imaging technique by detecting photoacoustic (PA) signal of a phantom in a highly scattering medium [2]. In the past decade, PAI has been developed rapidly for various medical applications, including detection of early stage breast cancer, detection of prostate cancer, and measuring tissue properties and molecular content [3].

Photoacoustic imaging (PAI) is a hybrid imaging technique that combines many of the merits of both optical and ultrasonic imaging. In this technique, pulsed laser radiation illuminates a tissue. Absorption of photons by chromophores results in thermoelastic expansion, which generates pressure waves. Therefore, photons in the tissue will be converted into acoustic waves. Then, PA images are provided by listening, via acoustic sensors, to the optical structure of the tissue.

Conversion of light to sound brings several advantages. Firstly, acoustic scattering in tissue is about three orders of magnitude lower than optical scattering per unit path length [3]. Therefore, PAI enables high resolution imaging of biological structures with higher penetration depth than optical imaging using the same contrast. Secondly, compared to pure ultrasonic imaging, PAI can provide rich optical contrast by multispectral illumination due to the fact that chemical composition of tissues contains information in a wide range of spectra. Photoacoustic imaging is particularly suitable for imaging blood vessels due to high optical contrast existing between blood and surrounding tissues. Thirdly, PAI provides images with no unwanted background because of the existence of non-absorbing tissue components at a specific wavelength. Moreover, unlike other optical imaging techniques, PAI has the potential to image all molecules, since all molecules 
have light-absorbing properties at some wavelength. For example, fluorescence imaging can only image molecules that are fluorescent [3].

\subsubsection{Photoacoustic signal generation}

Photoacoustic imaging is based on optical excitation and acoustic detection. The lightabsorbing object to be imaged is illuminated, usually by nanosecond-pulsed laser radiation. Physical characteristic of the exposed object and the time scale of energy dissipation determine the required time scale, the thermal and stress relaxation times, over which the light must be delivered. The thermal relaxation time, $\tau_{t h}$, and the stress relaxation time, $\tau_{s}$, are described by:

$$
\begin{gathered}
\tau_{t h}=\frac{d_{c}^{2}}{\alpha_{t h}} \\
\tau_{s}=\frac{d_{c}}{v}
\end{gathered}
$$

where $\alpha_{t h}$ is the thermal diffusivity $\left(\mathrm{m}^{2} / \mathrm{s}\right), d_{c}$ is the characteristic dimension of the heated region, and $v$ is the speed of sound in the respective medium.

Absorption of photons by the exposed object creates heat and that causes temperature rise. The temperature rise, $T$, can be expressed as:

$$
T=\frac{\eta_{t h} \mu_{a} F}{\rho c_{V}}
$$

where $\eta_{t h}$ is the percentage of light energy converted into heat, $\mu_{a}$ is the optical absorption coefficient $\left(\mathrm{cm}^{-1}\right), F$ is the optical fluence, $\rho$ is the density, and $c_{V}$ is the 
specific heat capacity at constant volume [4]. Then, the temperature rise causes expansion and consequently a localized pressure change. The localized pressure change, $p_{0}$, is expressed as:

$$
p_{0}=\Gamma \eta_{t h} A=\Gamma \eta_{t h} \mu_{a} F
$$

where $\Gamma$ denotes the Grueneisen parameter, a dimensionless unit that relates the pressure increase to the deposited optical energy, and $A$ is the specific optical energy deposition $\left(\mathrm{J} / \mathrm{cm}^{3}\right)$.

In soft tissue imaging, $\Gamma$ and $\eta_{t h}$ are usually treated as constants, and thus initial pressure rise is proportional to the optical energy deposition [4].

\subsubsection{The photoacoustic wave}

The propagation of the PA wave is described by generalized wave equation:

$$
\left(\nabla^{2}-\frac{1}{v_{s}^{2}} \frac{\partial^{2}}{\partial t^{2}}\right) p(r, t)=\frac{\beta}{\kappa v_{s}^{2}} \frac{\partial^{2} T(r, t)}{\partial t^{2}}
$$

where $p(r, t)$ is the pressure at location $r$ and $t$, and $T$ is the temperature rise. The solution describing the resultant PA wave is found from the general solution to this wave equation expressed by:

$$
p(r, t)=\frac{1}{4 \pi v_{s}^{2}} \frac{\partial}{\partial t}\left[\frac{1}{v_{s} t} \int p_{0}(r) \delta\left(t-\frac{\left|r-r^{\prime}\right|}{v_{s}}\right) d r^{\prime}\right]
$$

The initial energy deposition inside an isotropic spherical object, with uniform optical absorption, generates a uniform pressure increase. The spherical source generates two equal PA waves due to inside and outside pressure increases. One spherical pressure 
wave travels inward and has a converging negative magnitude. The other spherical pressure wave, having a diverging positive magnitude, travels outward. The detector observes the positive pressure wave followed by the negative pressure wave, resulting in an N-shaped or bipolar pressure profile. Figure 1.1 shows the N-shaped pressure profile of an isotropic spherical object of radius $R_{0}$. The pressure profile measured as a function of time at a point, $r$, will have a bipolar shape.

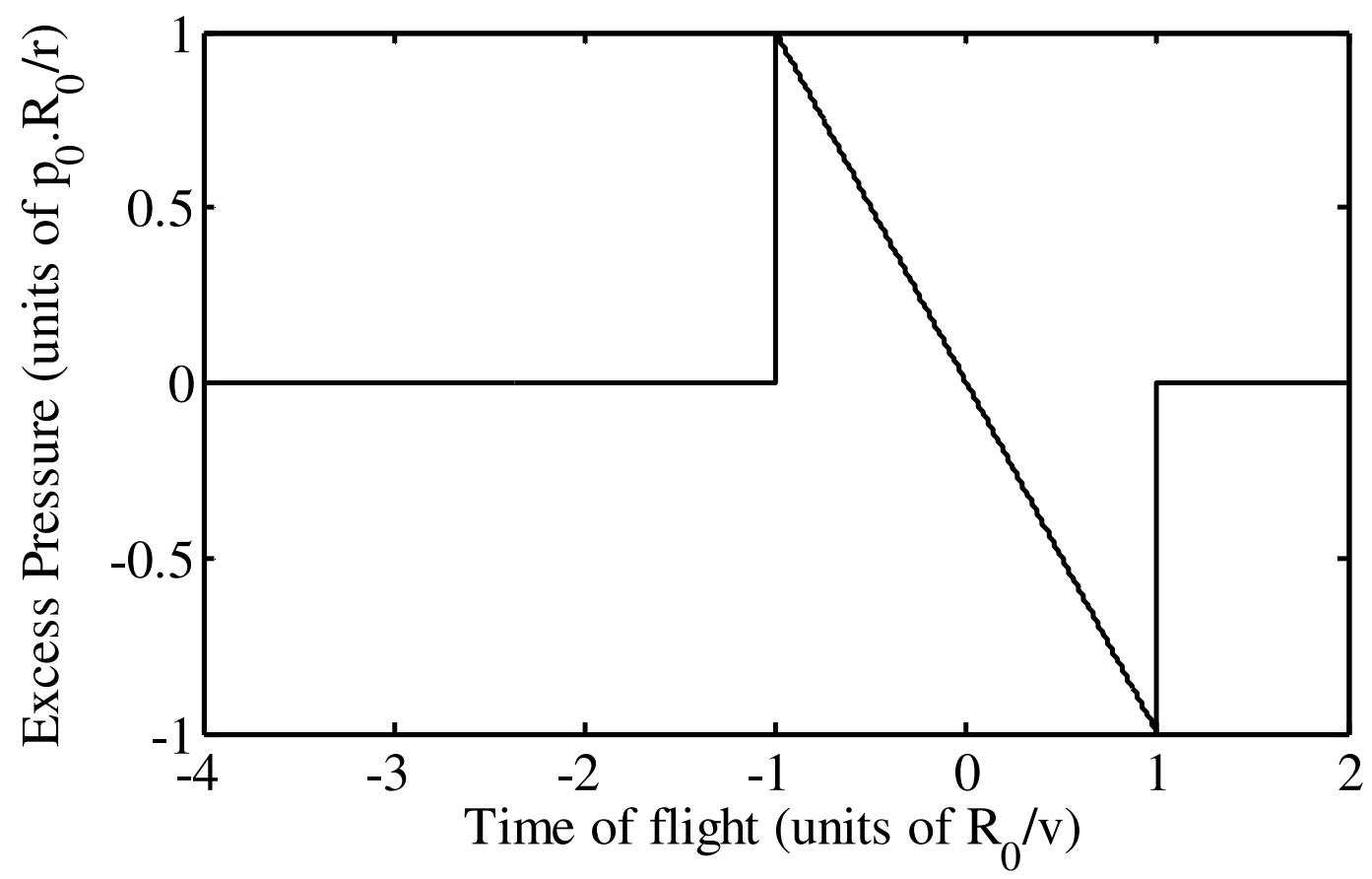

Figure 1.1. Photoacoustic signal profile in the time domain that contains the maximum amplitude of $P_{0} R_{0} / r$, and width equal to twice the quotient of $R_{0} / v$. The spherical source has a radius of $R_{0}$, and $V$ is the speed of sound in the propagating medium.

The temporal profile of the pressure increase due to a PA wave can be expressed as: 


$$
p(\tau)=\frac{\Gamma \mu_{a} F}{2 r}\left\{\begin{array}{cl}
-v \tau & |v \tau| \leq R_{0} \\
0 & |v \tau|>R_{0}
\end{array}\right.
$$

where $\tau=t-r / v$ is the time of flight and $\left(R-R_{0}\right)$ is the distance between the detector and the center of the spherical object.

The generated PA wave contains characteristic information of the light-absorbing object. Three characteristics can be derived from the generated PA signal. Time of arrival of the $\mathrm{N}$-shaped pressure profile indicates the distance between surface of the spherical object and the detector. The width of the PA signal (time difference between the abrupt edges), is related to the dimension of the sphere by the speed of sound in the propagating medium. The amplitude of the PA signal provides information about the optical absorption of the spherical PA source.

\subsection{Photoacoustic image reconstruction}

A variety of different PA image reconstruction techniques have been proposed including the spherical Radon transform [5], synthetic aperture beam forming [6], plane-wave approximation [7], iterative back projection [8,9], far-field approximation [10], compressed sensing [11,12], and singular-value decomposition (SVD) based algorithms $[13,14]$. The goal of any reconstruction modality is to create a map of the initial pressure distribution in a defined object space.

In our approach, we represent the system using a set of linear equations, described by

$$
g=\boldsymbol{H} \boldsymbol{f}+\boldsymbol{e}
$$


where $\boldsymbol{g}$ is a dataset of measured ultrasonic signals of the unknown target object, $\boldsymbol{H}$ is an imaging operator, $\boldsymbol{f}$ represents an estimate of the unknown target object, and $\boldsymbol{e}$ represents noise. In this imaging system, any object is represented by a series of cubic voxels in the spatial domain. The reconstruction technique contains the response of the system to a point source in each of the voxels in the object space and uses these responses to predict the position of an object. An experimental estimate of the imaging operator is acquired by measuring the system response to a PA point source that moves in a desired space grid.

\subsubsection{Pseudoinverse of the imaging operator}

An object, $f$, corresponding to a new set of imaging data, $g$, is then reconstructed using the following equation:

$$
\boldsymbol{f}=\boldsymbol{H}^{+} \boldsymbol{g}
$$

where $\boldsymbol{H}^{+}$is the pseudo-inverse of $\boldsymbol{H}$. The technique we chose for calculating the pseudoinverse utilizes SVD. The SVD of an $m \times n$ matrix, $\boldsymbol{H}$, is a factorization of the form

$$
\boldsymbol{H}=\boldsymbol{U} \boldsymbol{S} \boldsymbol{V}^{T}
$$

where $\boldsymbol{U}$ is the $m \times m$ unitary matrix of left-singular vectors of $\boldsymbol{H}$, the diagonal entries of $\boldsymbol{S}$ are the square root of the singular values of $\boldsymbol{H} \boldsymbol{H}^{\boldsymbol{T}}, \boldsymbol{V}$ is the $n \times n$ unitary matrix of right-singular vectors of $\boldsymbol{H}$, and ${ }^{T}$ represents a transpose operator [15].

The pseudo inverse of matrix $\boldsymbol{H}$ is then:

$$
\boldsymbol{H}^{+}=V \boldsymbol{S}^{-1} \boldsymbol{U}^{T}
$$


where $\boldsymbol{S}^{\mathbf{1}}$ is formed by replacing all the non-zero diagonal entries in $\boldsymbol{S}$ by their reciprocal.

\subsubsection{Regularization of the imaging operator}

As mentioned earlier, noise is present in the PA system of linear equations. Moreover, the matrix $\boldsymbol{H}$ is generally ill-conditioned. To remedy these issues, the pseudo-inverse calculated using SVD needs to be regularized. We have used a basic regularized SVD for this purpose. In regularized SVD, a specific number of the smallest singular values in $S$ are replaced by zero.

$$
\boldsymbol{S}=\operatorname{diag}\left(s_{p}, \ldots, s_{k+1}, s_{k}, \ldots, s_{i}, \ldots, 0\right)
$$

where $s_{p}>\ldots>s_{k}>\ldots>s_{i}>0$.

If $\boldsymbol{S}_{\boldsymbol{k}}$ represents the regularized equivalent of $\boldsymbol{S}$, and $\boldsymbol{H}_{\boldsymbol{k}}^{+}$is the regularized pseudoinverse, then by replacing $(\mathrm{k}-\mathrm{i})$ number of the smallest singular values by zero we have:

$$
\boldsymbol{S}_{\boldsymbol{k}}=\operatorname{diag}\left(s_{p}, \ldots, s_{k+1}, 0, \ldots, 0, \ldots, 0\right)
$$

and then we have:

$$
H_{k}^{+}=V S_{k}^{-1} U^{T}
$$

\subsection{Photoacoustic imaging approaches}

A variety of different approaches have been developed for PAI. These approaches are different in detection scheme and/or image reconstruction algorithm. Each approach 
employs a different number of transducers in scanning methods, staring methods, or scanning-staring methods [16]. The photoacoustic tomography (PAT) system employs a single transducer element that is mechanically scanned along the detection surface in one or two dimensions to create 2D or 3D images of the sample [17-21]. In staring methods, a 2D array of transducer elements, or a curved array of focused transducer elements is utilized for 2D or 3D PAI, and no scanning is necessary [9,14,16,22-29]. In a combined scanning-staring approach a linear array of transducer elements is scanned in one dimension for 3D PAI [30].

\subsection{Photoacoustic imaging of the brain}

Utilizing PAI for brain imaging is challenging due to the presence of the skull layer. The skull bone scatters light and severely attenuates and distorts ultrasonic signals. Hence, the skull layer is expected to deteriorate the accuracy of PA images. Thinner and softer skull bones found in newborns, with an average thickness of about $1.3 \mathrm{~mm}$ [32], result in less distortion and attenuation.

Photoacoustic tomography has proven successful for structural, functional, and molecular brain imaging in small animals [19,33-40]. This technique has been used to image brain structure, brain lesions, cerebral hemodynamic responses to hyperoxia and hypoxia, and cerebral cortical responses to neural activities induced by whisker stimulation in rats [33]. Moreover, in-vivo photoacoustic images of blood perfusion on skull cartilage of albino rats have been constructed [41]. 
Functional and molecular imaging is useful for tumor diagnosis [42]. Photoacoustic imaging was applied to image parameters such as oxygen saturation $\left(\mathrm{SO}_{2}\right)$, and total hemoglobin concentration (HbT) in brain tumor vasculature of the mouse [42]. These images are acquired based on the absorption spectra of oxygenated-hemoglobin $\left(\mathrm{HbO}_{2}\right)$ and deoxygenated-hemoglobin $(\mathrm{Hb})$.

$$
\begin{aligned}
\mathrm{SO}_{2} & =\frac{\left[\mathrm{HbO}_{2}\right]}{\left[\mathrm{HbO}_{2}\right]+[\mathrm{Hb}]} \\
\boldsymbol{H b T} & =\left[\boldsymbol{H b O}_{2}\right]+[\boldsymbol{H b}]
\end{aligned}
$$

where $[\mathrm{Hb}]$ and $\left[\mathrm{HbO}_{2}\right]$ are respectively the molar concentration of deoxygenatedhemoglobin and oxygenated-hemoglobin.

Optical contrast agents have also been used to improve the visibility of neurovascular images $[19,43,44]$. Spectroscopic photoacoustic tomography was utilized to image the contrast agent that targeted a brain tumor in a nude mouse through the intact scalp and skull [19].

The application of PAT in brain imaging has been extended to large animals with thicker skull bones as well $[45,46]$. The vascular structures of a monkey brain with an approximately 2-mm-thick skull through the intact scalp and skull bone were identified by PAT. Without the intact skull, the main structures of the exposed brain cortex were observable by PAT with greater clarity than with skull in place [45]. 
The feasibility of imaging through an infant cadaver skull has also been investigated. This study showed the capability of PAI in measuring and monitoring changes in blood oxygenation level through an infant skull. Photoacoustic images of three simulated vessels in a gel phantom embedded approximately $2 \mathrm{~mm}$ beneath the infant skull were captured. These images matched well with the photographs of the vessels $[32,47]$.

Recently, photoacoustic tomography through a human adult cadaver skull was investigated [48]. Photoacoustic tomography of a canine brain cortex through the whole human skull was captured. A photon recycler was designed and utilized to transmit more light through the skull by reflecting back-scattered photons to human skull, which enhanced the signal to noise ratio by a factor of 2.4. To enhance ultrasonic effects of the skull, surviving PA signals through the skull were passed through a high-pass filter with a cut-off frequency of about $100 \mathrm{kHz}$. This was done because high frequency ultrasonic signals are more attenuated by the skull. After processing and filtering the image, features of the canine brain were proved to be clearly identifiable. Eight main blood vessels were identified in the PAT image of the canine cortex. The experimental results showed that light can penetrate through the thick human skull (ranging from $\sim 7 \mathrm{~mm}$ (temporal area) to $\sim 11 \mathrm{~mm}$ in thickness) and that the produced PA signals are sufficiently detectable.

\subsection{Periventricular leukomalacia}

Periventricular leukomalacia (PVL) refers to ischemic damage to the white matter around the ventricles [49-51]. This neuropathologic lesion has emerged as the principal form of brain injury in premature infants. It is characterized by focal cystic lesions in deep white matter and a less severe but more diffuse non-cystic form throughout white matter 
[49,52]. The principal clinicopathologic correlate of PVL are spastic diplegia, in which motor impairment is greater in the legs than in the arms, and cognitive and behavioral deficits $[51,53]$.

One of the main factors in pathogenesis of PVL is an incomplete state of development of the vascular supply to the cerebral white matter which underlies a propensity for the occurrence of cerebral ischemia. Vascular supply to the cerebral white matter consists of the long and short penetrating arteries (Figure 1.2). The focal component of PVL occurs in the distribution of the end zones of the long penetrating arteries, while the diffuse component of PVL occurs in the distribution of 1) the border zones in between the individual long penetrating arteries and 2) the end zones of the short penetrating arteries. The long penetrating arteries are not fully developed in premature infants. The short penetrating arteries develop when the cerebral cortex fully develops in the post-term period $[49,52,53]$.

Clinical studies show that there is a relation between impaired cerebral blood flow (CBF) and the occurrence of PVL. Declines in CBF in long penetrating arteries usually indicate severe ischemia and focal PVL. Moreover, moderate ischemia and diffuse PVL would be expected when declines in $\mathrm{CBF}$ in short penetrating arteries are observed. On the other hand, changes in the concentrations of oxygenated and deoxygenated hemoglobin provide information about $\mathrm{CBF}$ and consequently the risk of PVL or other severe lesions in infants [49]. 


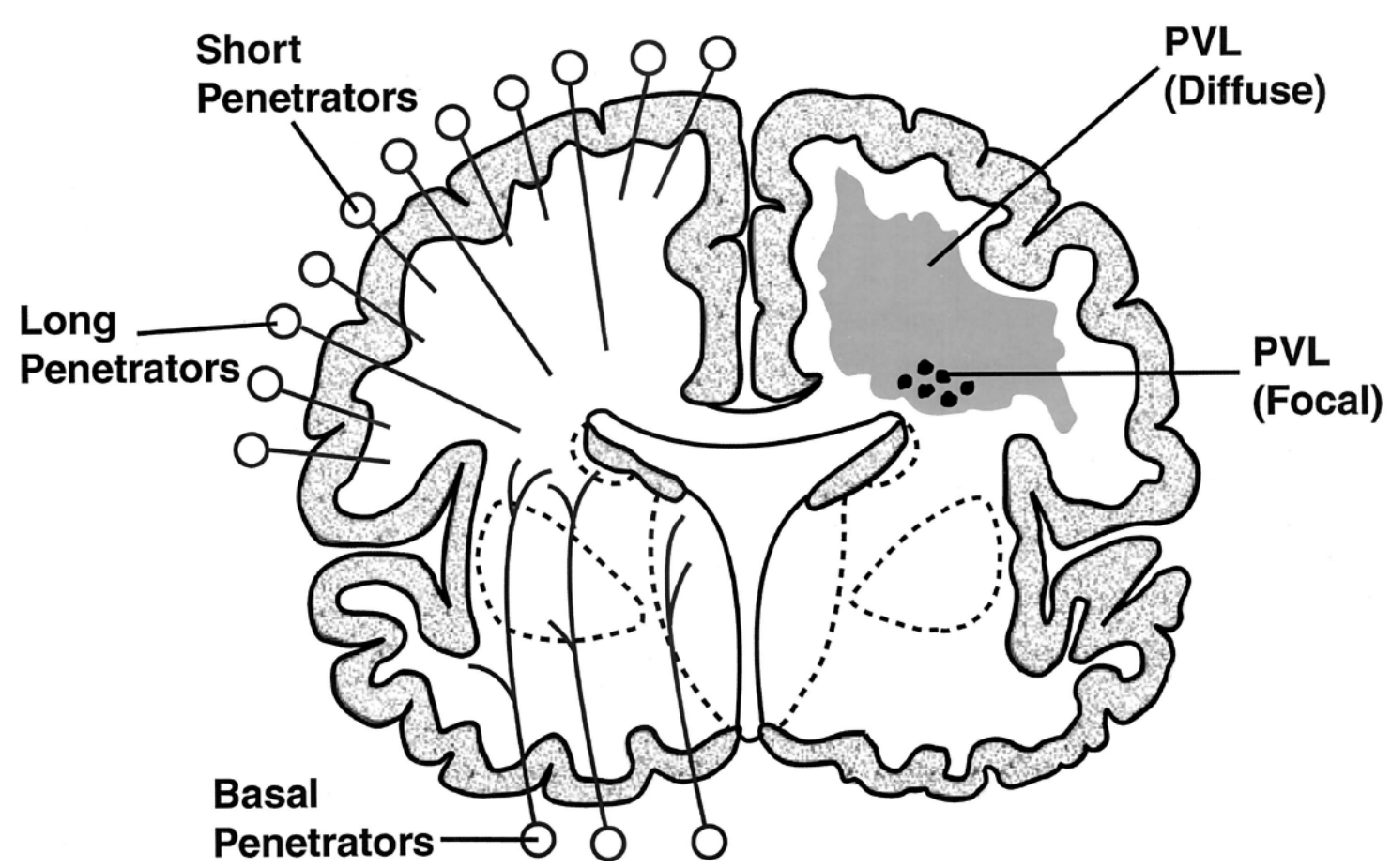

Figure 1.2. Coronal section of cerebrum. In one hemisphere focal and diffuse components of PVL are shown and in the other hemisphere vascular supply to the cerebral white matter is shown (adapted from Ref. [49]).

In response to hypoxic ischemic brain injury, the pre-myelinating oligodendrocytes in periventricular white matter undergo necrosis, resulting in PVL [50]. Ultrasonography can be used to identify focal cystic PVL $[49,52,53]$. Figure 1.3 shows sagittal and coronal ultrasonic images of an infant brain acquired 7 weeks after birth. In these images, cystic PVL in the pre-term infant born at 31 weeks is indicated by arrows [50]. However, ultrasonography is relatively insensitive to the diffuse form of PVL in the neonatal period $[49,52,53]$. Diffuse white matter injury appears to be much more common than cystic PVL [52,53]. 
Diagnosis of the latter typically requires diffusion-weighted magnetic resonance imaging (MRI) because of its advantageous high sensitivity. However, the use of MRI is limited by the need to transport fragile patients from the neonatal intensive care unit to imaging units [49-54].

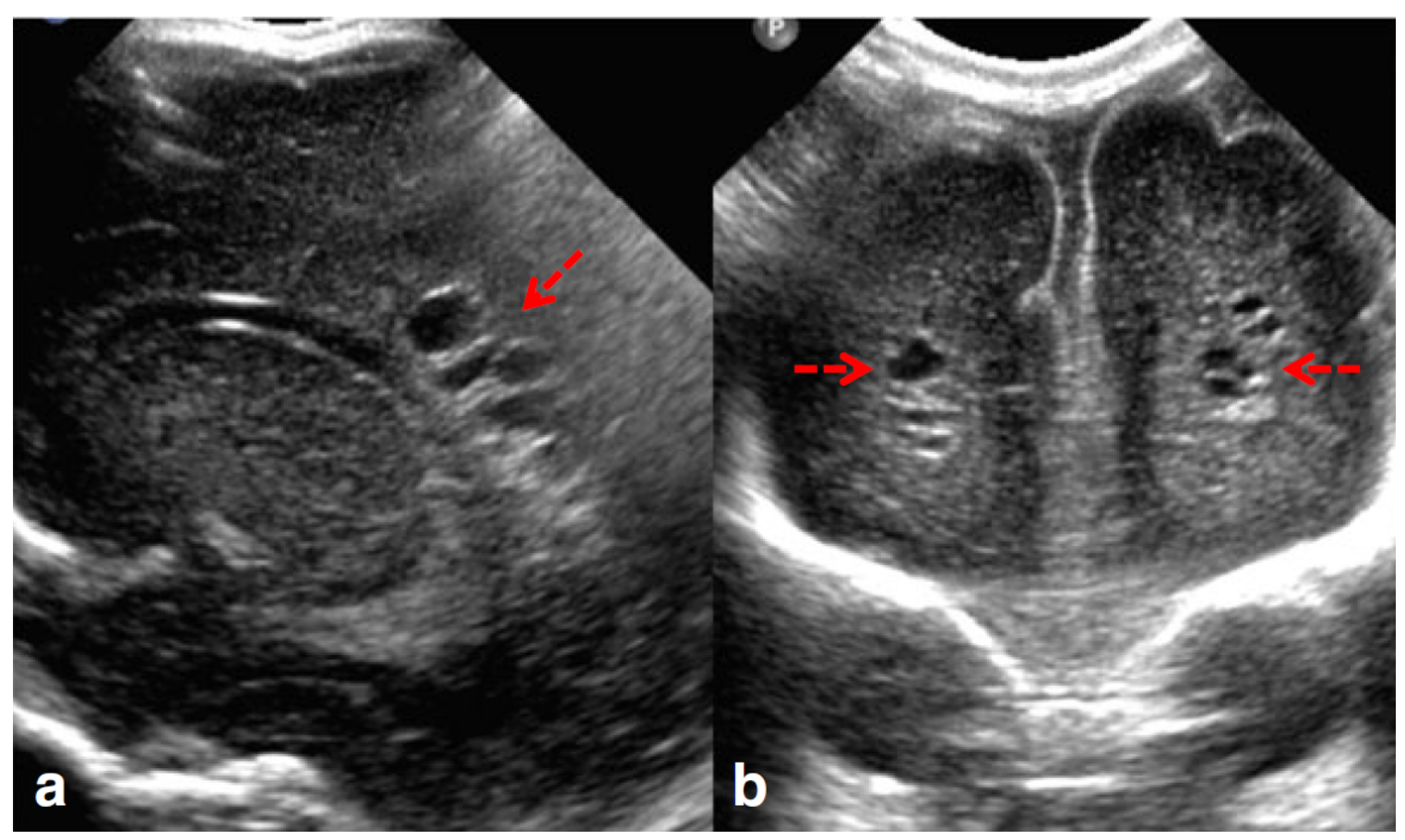

Figure 1.3. Cystic periventricular leukomalacia. (a) Sagittal and (b) coronal ultrasound images show cystic PVL (arrows). These images were acquired 7 weeks after birth of a preterm infant with 31 weeks gestational age (adapted from Ref. [50]).

Photoacoustic imaging has been proposed as a non-invasive technique for imaging neonatal brain injuries $[32,33]$. Since PAI uses both light and ultrasound, it is expected to be more sensitive to the tissue changes associated with diffuse PVL than ultrasonography [26,55]. Utilizing PAI to acquire anatomical image of non-cystic PVL, or the oxygen 
saturation changes associated with non-cystic PVL in neonatal brain is the aim of this project.

\subsection{Acoustic properties of the neonatal skull bone}

The acoustic properties (attenuation and reflection loss) of neonatal skull bone are presented in this section. Ultrasonic properties of the skull bone are mostly related to the skull thickness and skull density. Skull thickness and skull density vary by age, sex and race [56]. Acoustic insertion loss of the neonatal skull is less than that of the adult skull since the skull of an infant is thinner and softer. Acoustic insertion loss includes the effects of both ultrasonic attenuation through the skull and ultrasonic reflection at the surface of the skull.

To study ultrasonic insertion loss of the infant skull, the Xueding Wang et al. group used an ultrasound pulse-receiver (PR5072, Panametrics) to generate a broadband ultrasound pulse. The pulse was transmitted by a broadband transducer (V312,Panametrics), and was received by a needle hydrophone (HNV-1000, Onda) after about $2 \mathrm{~cm}$ of propagation. The calibrated needle hydrophone was opposite to and coaxially aligned with the V312 transducer. Measurements were made in the frequency range of $1 \mathrm{MHz}$ to $20 \mathrm{MHz}$ [47].

Frequency-dependent ultrasonic insertion loss of an infant skull bone is presented in Figure 1.4 (adapter from Ref [47]). Ultrasonic signals propagating through the skull bone attenuate more greatly at higher frequencies. Insertion loss started from $4 \mathrm{~dB}$ at $1 \mathrm{MHz}$, and increased consistently with the frequency. At $20 \mathrm{MHz}$, around $2 \%$ of the energy was still transmitted through the skull and reached the ultrasonic detector. 


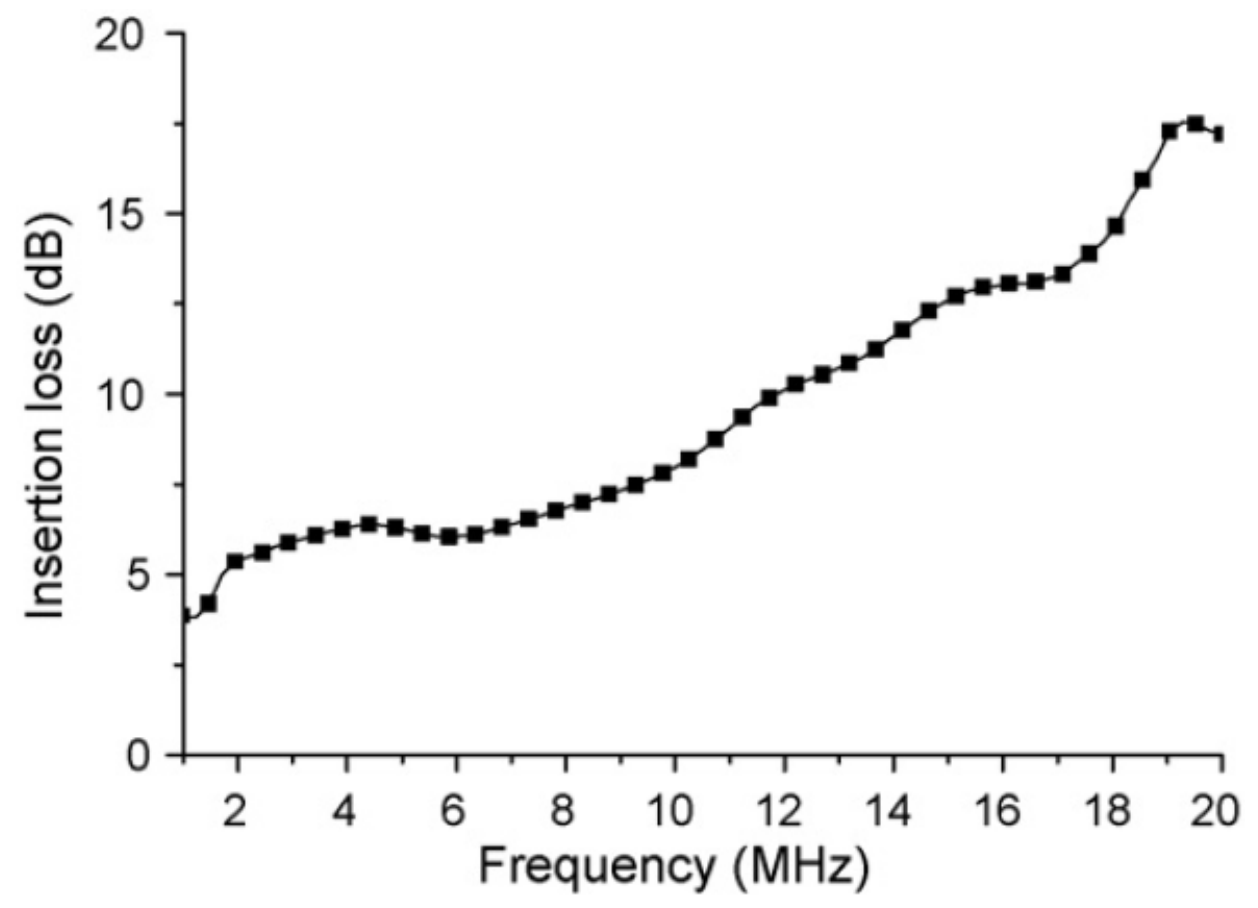

Figure 1.4. Ultrasound insertion loss of an infant skull as a function of frequency (adapted from Ref. [32])

\subsection{Light attenuation through the neonatal skull bone}

The skull attenuates light significantly due to its strong optical scattering properties. In two studies, the optical attenuation of an infant skull was evaluated using a laser and a photodetector (DET100, Thorlabs) [32,47]. The fluence of transmitted light through the skull was measured and normalized by the fluence of the incident light measured in the absence of the skull. The laser wavelength was tuned from $680 \mathrm{~nm}$ to $950 \mathrm{~nm}$ with a constant interval of $5 \mathrm{~nm}$. As shown in Figure 1.5, light transmittance through the infant skull is only slightly wavelength dependent. Between $650 \mathrm{~nm}$ to $950 \mathrm{~nm}$ the transmittance is fairly flat. About $60-70 \%$ of the light energy is transmitted through the infant skull $[32,47]$. 


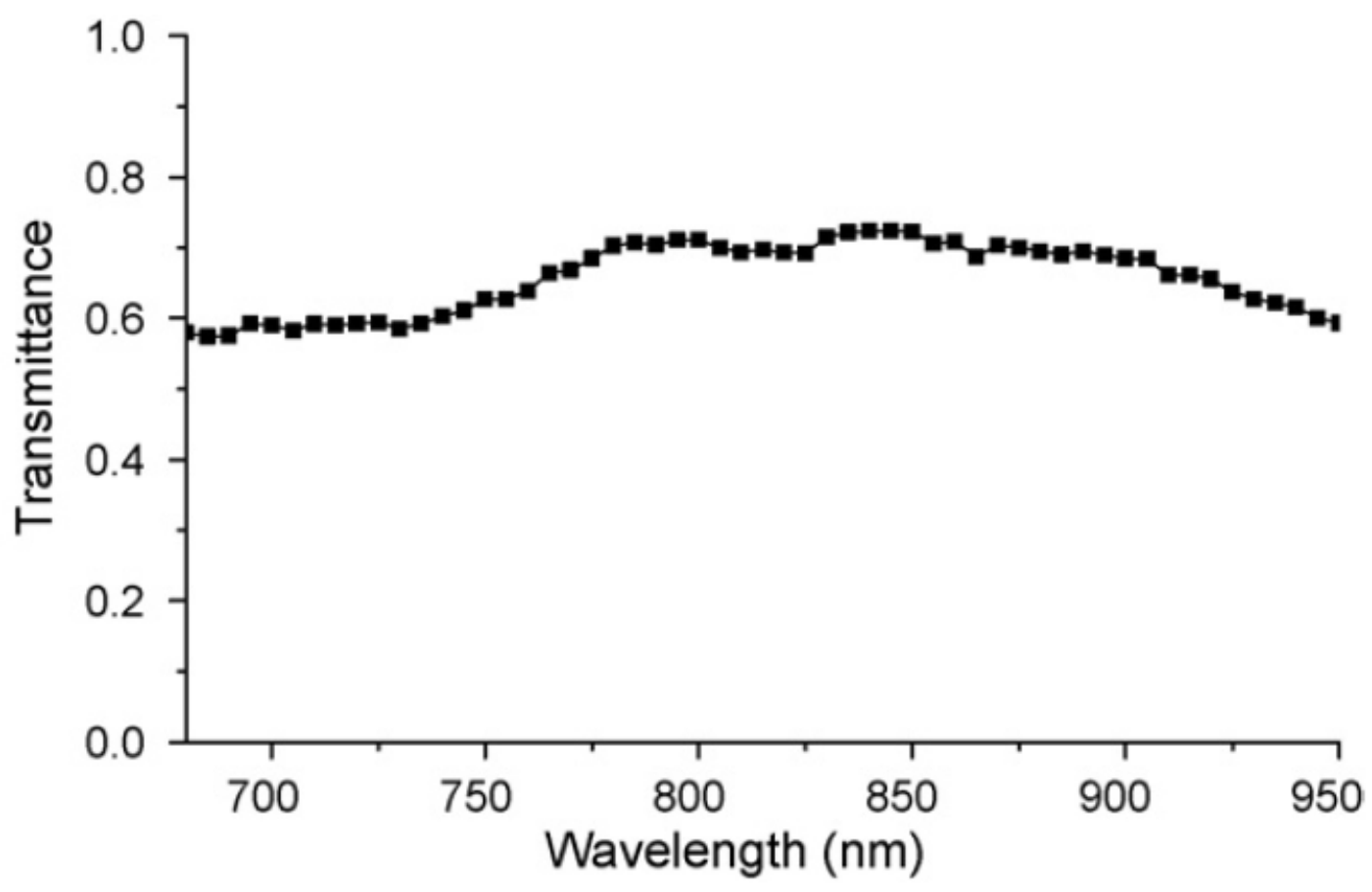

Figure 1.5. Light transmittance through the infant skull as a function wavelength. (adapted from Ref. [32])

\subsection{Motivation and objectives}

The PAI technique has not yet been used for brain imaging in neonates. Based on the literature review presented in the previous sections, a highly sensitive method that produces real-time 3D images of neonatal brain could be very helpful in diagnosis of the diffuse component of PVL. Since the main obstacle to propagation of PA signals is the skull, our approach was to investigate the possibility of imaging through a skullmimicking phantom. 


\subsubsection{Thesis objective and scope:}

The objective of this thesis was to utilize an acoustically realistic skull phantom to estimate the effect of premature infant skull on the propagation of PA signals and to determine imaging capability in the presence of the skull phantom. The material presented in the thesis falls into four main categories:

1) A skull phantom was constructed that mimicked the ultrasonic properties of neonatal skull bone to assess PAI for brain imaging in premature infants.

2) The propagation of PA signals through the skull phantom and the effects of the skull on the signals were studied.

3) The possibility of imaging through a skull-mimicking phantom was investigated. Photoacoustic images of some objects through the skull were acquired.

4) Two image enhancement methods were proposed in order to remove the effects of the skull from the PA signals, and consequently the PA images. The methods were shown to be successful in reducing artifacts from the images that were captured through the skull.

\subsubsection{Research approach}

In the research, a material proper for mimicking neonatal skull was identified, a skullmimicking phantom was constructed, the effects of the skull on the propagation of PA signals was investigated, the possibility of imaging through the skull phantom was studied using an experimental PAI setup, the effects of the skull on the PA signals and PA images were removed, and finally the results were shown. 
To find a material that properly mimics the behavior of the skull bone, acoustic insertion loss of candidate materials was measured and compared to the acoustic insertion loss properties of neonatal skull bone reported in previous sections. We selected a material that closely mimicked the ultrasonic properties of the neonatal skull bone. Then, we constructed a mold of a neonatal skull modeled with CAD software and using the head of a fairly realistic neonatal doll. Skull phantoms with different thicknesses and different shapes were constructed and tested.

Then, we measured the signals from a PA point source with and without the intervening skull phantom. The photoacoustic point source was tested at a multitude of grid locations to obtain the imaging operator for the transducer array with and without the skull phantom. The imaging operators were used to compute time delay and amplitude ratio maps representative of the effect of the skull phantom on PA signal propagation.

Then, imaging through a skull-mimicking phantom was investigated. We captured 3D images of some phantoms filled with indocyanine green in the presence of the skull phantom, which proved that the system was able to capture images of vessel-like targets through a layer with acoustic properties similar to the neonatal skull.

Finally, we proposed two image processing methods to eliminate the effects of the skull on the PA images of the objects. These methods were tested by performing another set of imaging experiments: Photoacoustic images of a light-absorbing rod were captured through the skull. The methods proved to be successful in reducing noise from the PA images of the rod 


\section{References}

1. T. Bowen, "Radiation-Induced Thermoacoustic Soft Tissue Imaging," IEEE Trans. Sonics Ultrason. 29, 187 (1982).

2. R. A. Kruger, "Photoacoustic Ultrasound," Medical Physics. 21, 127-131 (1994).

3. L. V. Wang and S. Hu, "Photoacoustic Tomography: In Vivo Imaging from Organelles to Organs," Science 335, 1458-1462 (2012).

4. L. V. Wang, "Photoacoustic Imaging and Spectroscopy", CRC, Boca Raton, 2009.

5. P. Liu, "Image reconstruction from photoacoustic pressure signals," Proc. SPIE 2681, 285-296 (1996).

6. C. G. Hoelen and F. F. de Mul, "Image reconstruction for photoacoustic scanning of tissue structures," Appl. Opt. 39, 5872-5883 (2000).

7. K. Kostli, D. Frauchiger, J. Niederhauser, and et al., "Optoacoustic imaging using a three-dimensional reconstruction algorithm," IEEE Journal of Quantum Electronics 7, 918-923 (2001).

8. G. Paltauf, J. A. Viator, S. A. Prahl, and S. L. Jacques, "Iterative reconstruction algorithm for optoacoustic imaging," J. Acoust. Soc. Am. 112, 1536-1544 (2002).

9. P. Ephrat, L. Keenliside, A. Seabrook, F. S. Prato, and J. J. Carson, "Threedimensional photoacoustic imaging by sparse-array detection and iterative image reconstruction," J. Biomed. Opt. 13, 054052 (2008).

10. P. Burgholzer, G. J. Matt, M. Haltmeier, and G. Paltauf, "Exact and approximative imaging methods for photoacoustic tomography using an arbitrary detection surface," Phys. Rev. E. Stat. Nonlin Soft Matter Phys. 75, 046706 (2007).

11. Z. Guo, C. Li, L. Song, and L. V. Wang, "Compressed sensing in photoacoustic tomography in vivo," J. Biomed. Opt. 15, 021311 (2010). 
12. J. Provost and F. Lesage, "The application of compressed sensing for photo-acoustic tomography," IEEE Trans. Med. Imaging 28, 585-594 (2009).

13. D. Modgil, B. E. Treeby, and P. J. La Riviere, "Photoacoustic image reconstruction in an attenuating medium using singular-value decomposition," J. Biomed. Opt. 17, 061204 (2012).

14. M. Roumeliotis, R. Stodilka, M. Anastasio, G. Chaudhary, H. Al-Aabed., E. Ng, A. Immucci, and J. J. L. Carson, "Analysis of a photoacoustic imaging system by the crosstalk matrix and singular value decomposition," Opt.Express 18, 11406-11417 (2010).

15. G. H. Golub and W. Kahan, "Calculating the singular values and pseudo-inverse of a matrix," SIMA Journal of Applied Mathematics 2, 205-224 (1965).

16. P. Ephrat and J. J. L. Carson, "Measurement of photoacoustic detector sensitivity distribution by robotic source placement," Proc. SPIE 6856, 85610 (2008).

17. D. Razansky, C. Vinegoni, and V. Ntziachristos, "Multispectral photoacoustic imaging of fluorochromes in small animals," Opt. Lett. 32, 2891-2893 (2007).

18. Y. Lao, D. Xing, S. Yang, and L. Xiang, "Noninvasive photoacoustic imaging of the developing vasculature during early tumor growth," Phys. Med. Biol. 53, 4203-4212 (2008).

19. M. Li, J. Oh, X. Xie, G. Ku, W. Wang, C. Li, G. Lungu, G. Stoica, and L. V. Wang, "Simultaneous molecular and hypoxia imaging of brain tumors in vivo using spectroscopic photoacoustic tomography," Proc IEEE 96, 481-489 (2008).

20. H. F. Zhang, K. Maslov, G. Stoica, and L. V. Wang, "Functional photoacoustic microscopy for high-resolution and noninvasive in vivo imaging," Nat. Biotechnol. 24, 848-851 (2006).

21. C. G. Hoelen, F. F. de Mul, R. Pongers, and A. Dekker, "Three-dimensional photoacoustic imaging of blood vessels in tissue," Opt. Lett. 23, 648-650 (1998). 
22. D. Piras, W. Xia, W. Steenbergen, and et al., "Photoacoustic Imaging of the Breast Using the Twente Photoacoustic Mammoscope: Present Status and Future Perspectives," IEEE Journal of Quantum Electronics 16, 730-739 (2010).

23. S. R. Kothapalli, T. J. Ma, S. Vaithilingam, O. Oralkan, B. T. Khuri-Yakub, and S. S. Gambhir, "Deep tissue photoacoustic imaging using a miniaturized 2-D capacitive micromachined ultrasonic transducer array," IEEE Trans. Biomed. Eng. 59, 1199-1204 (2012).

24. S. Vaithilingam, T. J. Ma, Y. Furukawa, I. O. Wygant, X. Zhuang, A. De La Zerda, O. Oralkan, A. Kamaya, S. S. Gambhir, R. B. Jeffrey Jr, and B. T. Khuri-Yakub, "Threedimensional photoacoustic imaging using a two-dimensional CMUT array," IEEE Trans. Ultrason. Ferroelectr. Freq. Control 56, 2411-2419 (2009).

25. S. A. Ermilov, T. Khamapirad, A. Conjusteau, M. H. Leonard, R. Lacewell, K. Mehta, T. Miller, and A. A. Oraevsky, "Laser optoacoustic imaging system for detection of breast cancer," J. Biomed. Opt. 14, 024007 (2009).

26. P. Ephrat, M. Roumeliotis, F. S. Prato, and J. J. Carson, "Four-dimensional photoacoustic imaging of moving targets," Opt. Express 16, 21570-21581 (2008).

27. M. Roumeliotis, P. Ephrat, J. Patrick, and J. J. L. Carson, "Development and characterization of an omni-directional photoacoustic point source for calibration of a staring 3D photoacoustic imaging system," Opt.Express 17, 15228-15238 (2009).

28. P. Ephrat, G. C. Albert, M. B. Roumeliotis, F. S. Prato, and J. J. L. Carson, "Localization of spherical lesions in tumour-mimicking phantoms by 3D sparse array photoacoustic imaging," Med Phys 37, 1619-1628 (2010).

29. M. B. Roumeliotis, R. Z. Stodilka, M. A. Anastasio, E. Ng, and J. J. Carson, "Singular value decomposition analysis of a photoacoustic imaging system and 3D imaging at 0.7 FPS," Opt. Express 19, 13405-13417 (2011). 
30. R. A. Kruger, K. D. Miller, H. E. Reynolds, W. L. Kiser Jr, D. R. Reinecke, and G. A. Kruger, "Breast cancer in vivo: contrast enhancement with thermoacoustic CT at 434 MHz-feasibility study," Radiology 216, 279-283 (2000).

31. H. H. Barrett and H. Gifford, "Cone-beam tomography with discrete data sets," Phys. Med. Biol. 39, 451-476 (1994).

32. X. Wang, D. L. Chamberland, and G. Xi, "Noninvasive reflection mode photoacoustic imaging through infant skull toward imaging of neonatal brains," J. Neurosci. Methods 168, 412-421 (2008).

33. X. Wang, Y. Pang, G. Ku, X. Xie, G. Stoica, and L. Wang, "Noninvasive laserinduced photoacoustic tomography for structural and functional in vivo imaging of the brain," Nat. Biotechnol. 21, 803-806 (2003).

34. W. Lu, Q. Huang, G. Ku, X. Wen, M. Zhou, D. Guzatov, P. Brecht, R. Su, A. Oraevsky, L. V. Wang, and C. Li, "Photoacoustic imaging of living mouse brain vasculature using hollow gold nanospheres," Biomaterials 31, 2617-2626 (2010).

35. X. Yang, A. Maurudis, J. Gamelin, A. Aguirre, Q. Zhu, and L. V. Wang, "Photoacoustic tomography of small animal brain with a curved array transducer," J. Biomed. Opt. 14, 054007 (2009).

36. J. Gamelin, A. Aguirre, A. Maurudis, F. Huang, D. Castillo, L. V. Wang, and Q. Zhu, "Curved array photoacoustic tomographic system for small animal imaging," J. Biomed. Opt. 13, 024007 (2008).

37. C. Li and L. V. Wang, "Photoacoustic tomography of the mouse cerebral cortex with a high-numerical-aperture-based virtual point detector," J. Biomed. Opt. 14, 024047 (2009).

38. J. Yao, J. Xia, K. I. Maslov, M. Nasiriavanaki, V. Tsytsarev, A. V. Demchenko, and L. V. Wang, "Noninvasive photoacoustic computed tomography of mouse brain metabolism in vivo," Neuroimage 64, 257-266 (2013). 
39. S. Hu and L. V. Wang, "Neurovascular photoacoustic tomography," Frontiers in Neuroenergetics 2, (2010).

40. X. Wang, Y. Pang, G. Ku, G. Stoica, and L. V. Wang, "Three-dimensional laserinduced photoacoustic tomography of mouse brain with the skin and skull intact," Opt. Lett. 28, 1739-1741 (2003).

41. M. C. Pilatou, E. Marani, F. F. de Mul, and W. Steenbergen, "Photoacoustic imaging of brain perfusion on albino rats by using evans blue as contrast agent," Arch. Physiol. Biochem. 111, 389-397 (2003).

42. J. Oh, M. Li, X. Xie, and et al., "In vivo functional photoacoustic imaging of brain tumor vasculature," SPIE, 7th Conference on Biomedical Thermoacoustics, Optoacoustics, and Acousto-Optics Location: San Jose, CA 6086, C860 (2006).

43. P. H. Wang, H. L. Liu, P. H. Hsu, C. Y. Lin, C. R. Wang, P. Y. Chen, K. C. Wei, T. C. Yen, and M. L. Li, "Gold-nanorod contrast-enhanced photoacoustic micro-imaging of focused-ultrasound induced blood-brain-barrier opening in a rat model," J. Biomed. Opt. 17, 061222 (2012).

44. J. Xia and L. V. Wang, "Photoacoustic tomography of the brain," in Optical Methods and Instrumentation in Brain Imaging and Therapy, S. J. Madsen, ed. (Springer, 2013), pp. 137-156.

45. X. Yang and L. V. Wang, "Monkey brain cortex imaging by photoacoustic tomography," J. Biomed. Opt. 13, 044009 (2008).

46. L. Nie, Z. Guo, and L. V. Wang, "Photoacoustic tomography of monkey brain using virtual point ultrasonic transducers," J. Biomed. Opt. 16, 076005 (2011).

47. X. Wang, J. B. Fowlkes, D. L. Chamberland, G. Xi, and P. L. Carson, "Reflection mode photoacoustic imaging through infant skull toward noninvasive imaging of neonatal brains," Proc. SPIE, Photons Plus Ultrasound: Imaging and Sensing 7177, 717709 (2009). 
48. L. Nie, X. Cai, K. Maslov, A. Garcia-Uribe, M. A. Anastasio, and L. V. Wang, "Photoacoustic tomography through a whole adult human skull with a photon recycler," 17, (2012).

49. J. J. Volpe, "Neurobiology of periventricular leukomalacia in the premature infant," Pediatr. Res. 50, 553-562 (2001).

50. C. A. Badve, P. C. Khanna, and G. E. Ishak, "Neonatal ischemic brain injury: what every radiologist needs to know," Pediatr. Radiol. 42, 606-619 (2012).

51. B. Y. Huang and M. Castillo, "Hypoxic-ischemic brain injury: imaging findings from birth to adulthood," Radiographics 28, 417-39; quiz 617 (2008).

52. J. J. Volpe, "Encephalopathy of prematurity includes neuronal abnormalities," Pediatrics 116, 221-225 (2005).

53. J. J. Volpe, "Cerebral white matter injury of the premature infant-more common than you think," Pediatrics 112, 176-180 (2003).

54. J. Fu, X. Xue, G. Fan, K. You, and Y. Ren, "Values of diffusion-weighted magnetic resonance imaging in the early detection of periventricular leukomalacia in preterm infants," Chinese journal of pediatrics 46, 354-358 (2008).

55. P. Tavakolian, R. Todd, I. Kosik, A. Chamson-Reig, F. Vasefi, K. S. Lawrence, and J. J. L. Carson, "Development of a neonatal skull phantom for photoacoustic imaging," Proc. SPIE. 8581, Photons Plus Ultrasound 8581, (2013).

56. F. J. Fry and J. E. Barger, "Acoustical properties of the human skull," J. Acoust. Soc. Am. 63, 1576-1590 (1978).

57. P. Tavakolian, I. Kosik, A. R. Chamson, K. S. Lawrence, and J. J. L. Carson, "Potential for photoacoustic imaging of the neonatal brain,"Proc. SPIE, Photons Plus Ultrasound 8581, (2013). 
58. J. Sadler, K. Shapoori, E. Malyarenko, A. DiCarlo, J. Dech, F. Severin, and R. G. Maev, "Resolving the Location of Acoustic Point Sources Scattered Due to the Presence of a Skull Phantom," 30, 271-278 (2011). 


\section{Chapter 2}

\section{Investigation of 3D photo acoustic neonatal brain imaging using a skull-mimicking phantom}

\subsection{Overview}

In this manuscript, we evaluated the effects of the skull bone on PA signals and images in

detail. A photoacoustic point source was raster-scanned within the imaging cavity of a 128-channel PAI system to capture the imaging operator with and without the intervening skull phantom layer. We captured 3D photoacoustic images of tubes filled with indocyanine green (ICG). The system was capable of reconstructing an image of a tube filled with $1.25 \mu \mathrm{M}$ ICG in the absence of the skull, but the ICG in the tube had to be increased to $50 \mu \mathrm{M}$ in order to reconstruct images of the tube when the intervening skull layer was present.

This chapter describes the method developed for fabrication of a skull-mimicking phantom. It also introduces the 128-channel PAI system with a spherical imaging cavity applied to image through a skull phantom. Explanations regarding making of synthetic objects that represent vessels are also included.

\subsection{Introduction}

\subsubsection{Background and motivation}

Imaging plays an important role in the diagnosis of neonatal brain injury [1]. Periventricular leukomalacia (PVL) refers to ischemic damage to the white matter around the ventricles. This neuropathologic lesion has emerged as the principal form of brain injury in premature infants. Clinical studies show that there is a relation between 
impaired cerebral blood flow (CBF) and the occurrence of PVL [1-3]. With declines in CBF in long penetrating arteries severe ischemia and focal PVL would be expected. Moreover, with declines in $\mathrm{CBF}$ in short penetrating arteries moderate ischemia and diffuse PVL would be expected. Changes in the concentrations of oxygenated and deoxygenated hemoglobin provide information about CBF and consequently the risk of PVL or other severe lesions in infants $[1,2]$.

Among all imaging modalities, ultrasonography remains the most easily accessible technique for neonatal brain imaging. Although this modality is sensitive for diagnosis of cystic PVL, it is relatively insensitive to less severe but more diffuse, and more common PVL [1,4]. Diagnosis of the noncystic PVL typically requires diffusion-weighted magnetic resonance imaging (MRI). The high sensitivity of MRI enables better visualization of white matter abnormalities at an earlier stage. However, the use of MRI is limited by the need to transport these fragile patients from the neonatal intensive care unit to imaging units. Therefore, more sensitive methods for detection of non-cystic PVL are needed. Since photoacoustic imaging (PAI) uses both light and ultrasound, it is expected to be more sensitive to the tissue changes associated with non-cystic PVL than ultrasonography. Utilizing PAI for acquiring anatomical image of neonatal brain, or acquiring information regarding oxygen saturation associated with non-cystic PVL in neonatal brain is the ultimate goal of this project. We worked on several technical challenges that needed to be overcome before the goal can be achieved.

Photoacoustic imaging has proven successful for structural, functional, and molecular brain imaging in small animals [5,6]. The feasibility of photoacoustic tomography (PAT) for imaging the primate brain through the relatively thick primate skull bone $(\sim 2 \mathrm{~mm})$ has 
been recently demonstrated [7]. Images of blood vessels have been captured through a cadaver infant skull using PAI [8,9]. For the first time, PAT of a canine brain through a cadaver adult human skull was studied by a group at Washington University. However, PAI through the skull continues to present a serious challenge to photoacoustic image reconstruction and is an area of active research. Since the skull attenuates and distorts ultrasound signals and scatters light strongly, it severely deteriorates images of the brain [10].

\subsubsection{Objective and approach}

In this work, our objective was to experimentally investigate the effect that the skull layer has on the propagation of PA signals with the aim of PAI through a skull layer. We evaluated a variety of skull mimicking materials and selected a material that closely mimicked the ultrasound insertion loss of neonatal skull bone. We constructed a mold representative of the frontal, parietal, and occipital bones of the cranium of a neonatal skull by creating a 3D CAD model of the head of a realistic infant doll. Skull phantoms of different thicknesses were fabricated with the mold. We measured the signals from a photoacoustic point source with and without the intervening skull phantom. The photoacoustic point source was tested at a multitude of grid locations to obtain imaging operators for the transducer array with and without the skull phantom. The imaging operators were used to compute time delay and amplitude-ratio maps representative of the effect of the skull phantom on photoacoustic signal propagation. Finally, we captured 3D images of tubes filled with indocyanine green (ICG) in the presence of the skull phantom, which demonstrated that PAI of vessel-like targets was possible through a layer 
with acoustic properties similar to the neonatal skull. Preliminary results of this work have been presented recently [11]' [12].

\subsection{Methodology}

\subsubsection{System setup}

The PAI system is shown in Figure 2.1 (a). The system included a spherical imaging cavity (transducer array) with an internal diameter of $9.6 \mathrm{~cm}$. The imaging cavity incorporated an array of 128 custom-built unfocused ultrasound transducers (center frequency of 2.7 MHz and bandwidth of 127\%). Two four-legged optical fibers (Lumen Dynamics, Missisauga, ON, Canada) were mounted to the top and bottom periphery of the array for optical illumination during imaging experiments. Each fiber optic cable was connected to the output of a tunable laser (OPO, Nd:YAG, Phocus, Opotek, Inc., Carlsbad, California). Each ultrasound transducer incorporated a preamplifier (gain 400X) in close proximity to the piezoelectric material. All 128 transducers were connected to a 128-channel data acquisition system (DAQ) (SonixDAQ, 40-MHz sampling rate, 12-bit resolution, Ultrasonix Medical Corp., Richmond, British Columbia) through a custom interface card that provided power to each transducer preamplifier. The transducer signals were captured, stored in internal memory of the DAQ, and transferred to a workstation via USB2.0.

For system calibration, a photoacoustic point source was constructed from a fiber optic cable $105 \mu \mathrm{m}$ in diameter. One end of the fiber was polished and coated in a black material (Connector coating, MG chemicals, Burlington, Canada) and attached to the arm of a robot (E2C2351S-UL, Epson RC+ software, ver. 4.1.0, Epson Corporation, CA, 
USA). The other end of the fiber was polished and stripped of cladding. The bare end was illuminated with light from a 1064-nm pulsed laser (Surelite I-20, Nd:YAG, Continuum, Santa Clara, California). The laser Q-switch specifies the energy of the pulsed output beam. In order to have suitable output laser energy, an experiment was performed to select a proper Q-switch value. For each calibration scan, the selected Q-switch provided photoacoustic amplitudes in the moderate range at all transducers. For all the calibration scans performed in this thesis, the Q-switch of the surelite laser was set to $355 \mu$ s.

\subsubsection{Image reconstruction}

In an earlier publication, our group introduced a technique for photoacoustic image reconstruction [13]. In this approach, image reconstruction was performed using a system of linear equations, described by

$$
g=H f+e
$$

where $\boldsymbol{g}$ is a measured data set of ultrasound signals, $\boldsymbol{H}$ is the imaging operator, and $\boldsymbol{f}$ represents an estimate of the unknown target object and $\boldsymbol{e}$ is a noise pattern. For reconstruction of photoacoustic images, an experimental estimate of the imaging operator was acquired by measuring the system response to a PA point source moving in a desired space grid. Image reconstruction and display was performed offline with custom code written in MATLAB® (The Mathworks, Inc., version 7.8.0, Natick, Massachusetts).

To capture the imaging operator of the 3D PAI system, the robot was used to raster scan the photoacoustic point source within the transducer array. After localizing the point source to each grid location, the $1064 \mathrm{~nm}$ pulsed laser was pulsed to illuminate the point

source. A digital delay generator (Continuum, Model TCU-1, Santa Clara, USA) was 
used to provide precise delays for triggering the lasers and the DAQ system. For the scan experiments performed in this paper the delays were set to $12.3 \mu \mathrm{s}$. The DAQ was triggered by the delay generator and photoacoustic signals from the point source were captured on all 128 transducers simultaneously and stored in memory. The robot was instructed to move the point source to an adjacent grid location and the process was repeated until the complete imaging operator was obtained. After all grid points had been sampled, the data stored on the DAQ was transferred to the workstation. Software used to coordinate the robot and the data acquisition hardware was custom written in LabVIEW (National Instruments ${ }^{\mathrm{TM}}$ ).

To track potential changes in laser pulse energy on the calibration scan, an ultrasound transducer (monitoring transducer) was used to record the PA signals of the point source at all grid points. This transducer was mounted next to the point source on the robot arm. The signal on the monitoring transducer was captured by the DAQ (along with all 127 other transducers) at each grid location. Time of arrival and maximum amplitude of the PA signal detected at monitoring transducer at each grid location with and without the skull are shown in Figure 2.1 (b) and (c). Additionally, the mean time of arrival, the standard deviation of the time of arrival, and the maximum PA amplitude are displayed. 
(a)

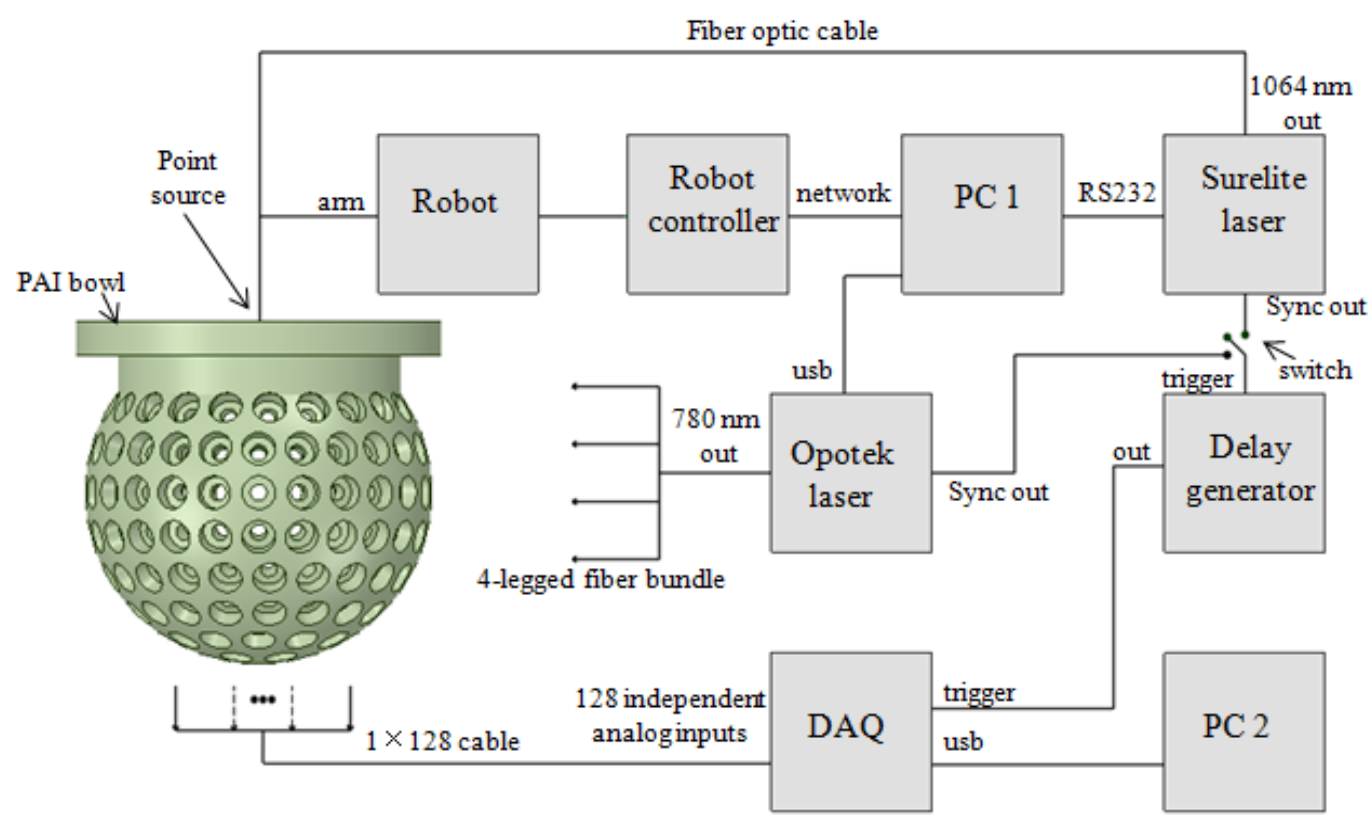

(b)

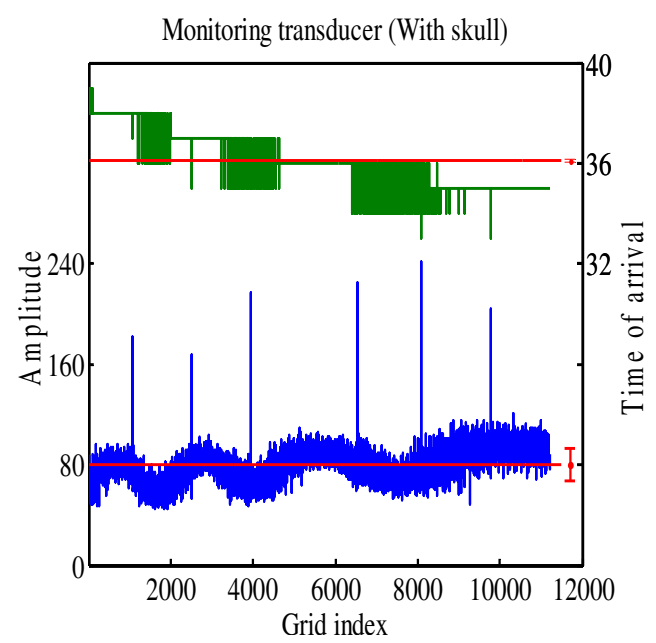

(c)

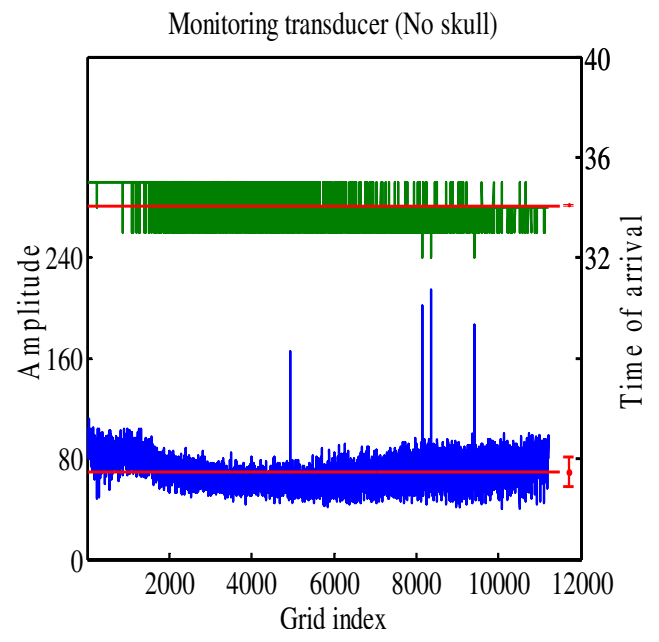

Figure 2.1. (a) Photoacoustic imaging system which includes a spherical imaging cavity consisting of 127 ultrasound transducers, a DAQ system, two lasers, a delay generator, two computers, a robot and its controller, and a photoacoustic point source attached to the robot; (b) Blue curve is the maximum amplitude of the PA signals detected at the monitoring transducer at all grid points for the calibration scan performed in the absence of the skull; green curve is the time of arrival of these 


\section{PA signals; red curves display the mean value and standard deviation of the blue and green curves. (c) The same parameters described in (b), this time for the calibration scan captured through the skull.}

\subsubsection{Imaging experiment}

\subsubsection{Skull-mimicking phantom}

In preliminary work, frequency-dependent ultrasound insertion loss for a variety of materials, including cured mixtures of epoxy and titanium powder has been reported [11]. Acoustic insertion loss included the effects of both ultrasound attenuation through the material, and ultrasound reflection at the surfaces of the material. Measurements revealed large differences between the acoustic properties of skull bone (as reported in Ref [8]) and materials such as polycarbonate sheet, acrylic sheet, and PVC sheet (data not shown).

Of all the materials tested, samples prepared from epoxy mixed with titanium dioxide powder were the best matched to the acoustic properties of neonatal skull bone. To finetune the properties of the epoxy-titanium dioxide mixtures, samples were prepared with different proportions of epoxy and titanium dioxide powder and different thicknesses. Ultrasound insertion loss across a range of frequencies $(0.9 \mathrm{MHz}$ to $3 \mathrm{MHz})$ for each of the samples was measured and compared with that of a neonatal skull bone which was reported by others (see Refs [8,9]). After performing these experiments, the optimal concentration of titanium dioxide powder in the mixture and the optimal thickness of the cured mixture were identified.

A skull mold was constructed based on the size of an infant's skull measured by an MRI image of its head. We have used the same 3D model of a hairless head of a realistic infant doll as we used in previous work [11]. The top part of the 3D head model was selected 
for making a skull mold. The skull model was placed on a cylindrical substrate with four screw holes. We also machined the bottom component of the skull mold by making a skull-shaped hole in a cylindrical block. The two halves of the mold were made such that a controlled gap would be created for the epoxy and titanium dioxide mixture.

The mold was CNC machined (Charlyrobot 2U, Charlyrobot company, Cruseilles, France) from two solid blocks of Teflon®. For machining, CAM software (Edgecam,Vero software) was used to create and translate a tool path to machine language. The three tool paths, created on the CAM software and performed on Teflon blocks, were roughing, profiling, and parallel lacing operations.

A skull phantom was made by curing the mixture of epoxy and titanium dioxide powder inside a mold. A mixture of 56\% epoxy and $44 \%$ titanium dioxide powder was poured into the mold. In order that the skull phantom was easily detachable from the skull mold, a release agent (Ease Release 300, Mann release technologies, Inc., USA) was sprayed on the surface of the mold before pouring the epoxy mixture into the mold. After two hours of the curing process, the skull phantom was taken out and left exposed to the air for a day to harden. The phantom thickness was heterogeneous with a thickness of $1.42 \mathrm{~mm}$ at the top, $1.63 \mathrm{~mm}$ at the front, and $1.53 \mathrm{~mm}$ at the back of the skull. Figure $2.2(\mathrm{a}, \mathrm{b}$, and c) shows the mold, a 3D CAD model of the skull phantom, and a skull phantom constructed from a mixture of epoxy and titanium dioxide powder.

To position the skull inside the transducer array, a holder was designed in a CAD software (Spaceclaim 2012+, Concord, Massachusetts,) and was 3D printed (BGB-3000, Bitsfrombytes, UK). As shown in Figure 2 (d), the skull phantom was positioned within 
the imaging cavity, with the top surface of the skull phantom facing the transducer array. Illumination was toward the concave part of the skull and transducers received signals from the convex part of the skull.

\subsubsection{ICG target phantom}

Two different experiments were performed to image vessel-like objects. One tube (I.D. $0.38 \mathrm{~mm}$, O.D $1.09 \mathrm{~mm}$, polyethylene tubing, Clay Adams, U.S.A) in one experiment and two tubes in another experiment were filled with indocyanine green (ICG) at one of 4 concentrations $(50,100,250,500$, or $1.25 \mu \mathrm{M})$. Experiments were repeated with and without the presence of the skull phantom. To compare images captured with and without the skull phantom, we placed the tubes at the same position and orientation in the imaging cavity. In each case, the tubes were inserted within the bulb of a transfer pipette to have both ends of the tubes fixed. Then, the pipette was attached to the robot arm. A syringe filled with ICG (at one of 4 concentrations) was injected into the tube during each imaging experiment. The tube was flushed with water several times between experiments.

\subsubsection{Imaging operator}

A calibration scan of the imaging system included 7 planes in the $z$ axis and 40 by 40 steps in the $\boldsymbol{x}$ and $\boldsymbol{y}$ directions. The scan was performed in both the absence and the presence of the skull within the transducer array. An imaging operator was obtained from the calibration scan, with a step size of $0.5 \mathrm{~mm}$ for the grid located near the center of the array, which covered a volume of $20 \mathrm{~mm} \times 20 \mathrm{~mm} \times 3.5 \mathrm{~mm}$. The transducer array and the skull phantom were filled with water for acoustic coupling. At each of the 11,200 grid locations, photoacoustic signals were recorded for approximately $25 \mu$ s from each of the 
128 transducers simultaneously. The point source was scanned across each $x$-coordinate from the lowest grid point to the highest, then the $y$-coordinate was incremented once; following this, the $x$-coordinate was decremented from the highest point to the lowest and the process was then repeated. The skull phantom was positioned within the hemispherical imaging cavity with a holder, as shown in Figure 2.2 (d). The same procedure was repeated with the skull in the imaging cavity, and the imaging operator was captured. Each scan took about an hour to complete. Each calibration scan resulted in a data set of approximately $2.8 \mathrm{~GB}$. For each calibration scan, the main user interface of the SonixDAQ was set to 1000 sample numbers, $50 \mu$ s line duration, $2 \mu$ s gain delay, 0 $\mathrm{mV}$ gain offset, $6 \mu$ s receive delay, with a digital DC filter, fixed TGC and minimum TGC curve, 4GB buffer size, and $40 \mathrm{MHz}$ sampling rate.

The imaging operator was processed to eliminate noise and to reduce the reconstruction time. Although each signal from each transducer includes 1000 sample points, the signals were down-sampled 4 times to 250 points during the preprocessing procedures. Thus, the imaging operator size, and consequently the reconstruction time, was reduced greatly without noticeably affecting the reconstruction results. Photoacoustic signals were rectified to provide non-negative signals. Then, a threshold of about 0.1 of the maximum amplitude of the PA signals was applied to eliminate low amplitude noise.

Figure 2.2 (d) displays 12 lines of the calibration scan in one $\boldsymbol{x y}$ plane, with the skull in place. The green lines represent the central lines of the scan at the middle $x y$ plane. The red lines (at the opposite side of the $\boldsymbol{x}$ axis) and the blue lines (along the $\boldsymbol{x}$ axis) represent the grid locations close to the front and back edges of the skull, respectively. Subsets of 
the imaging operator associated with these lines are displayed in Figure 2.4 and described in Section 3.

(a)

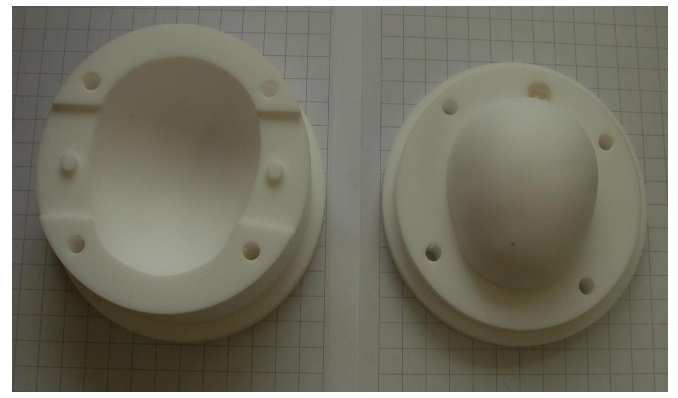

(c)

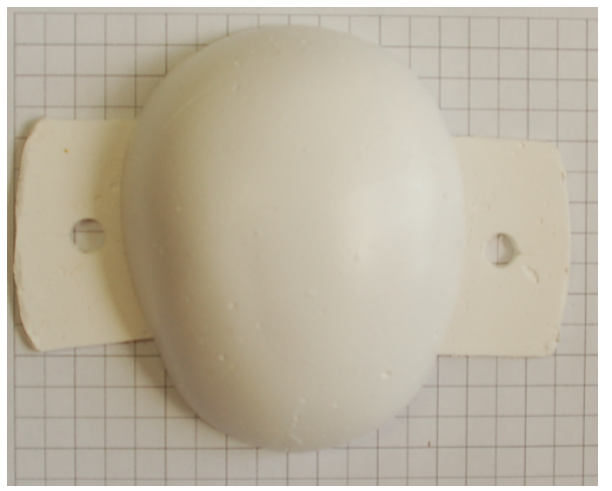

(e)

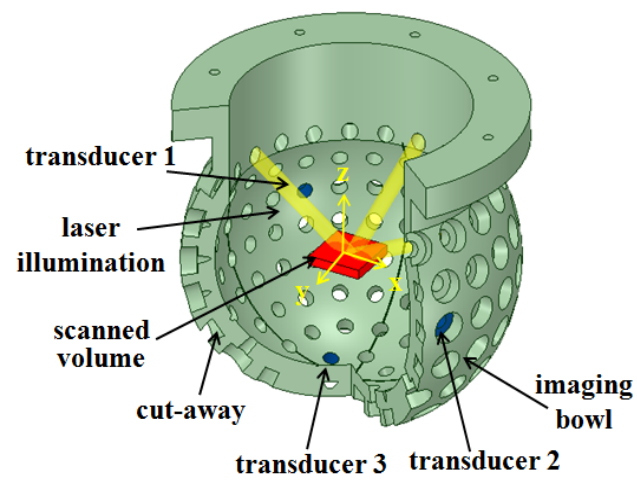

(b)

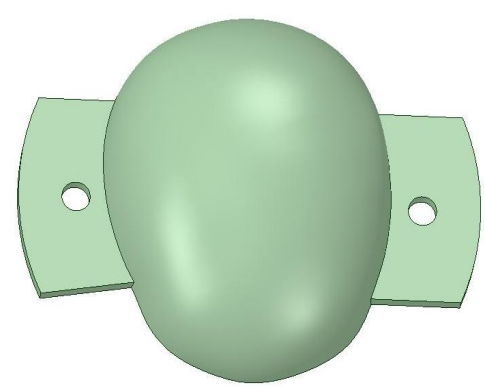

(d)

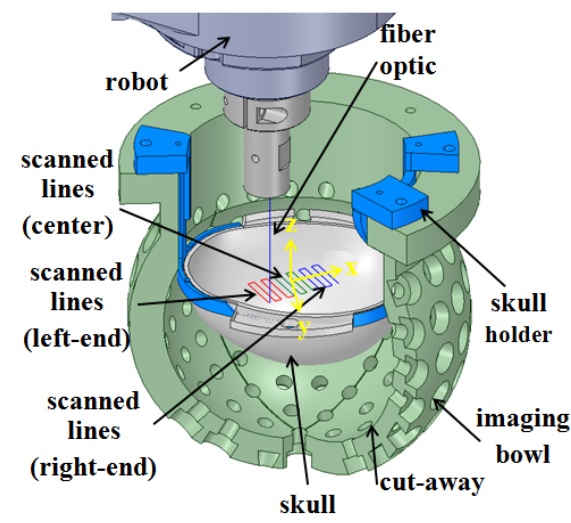

(f)

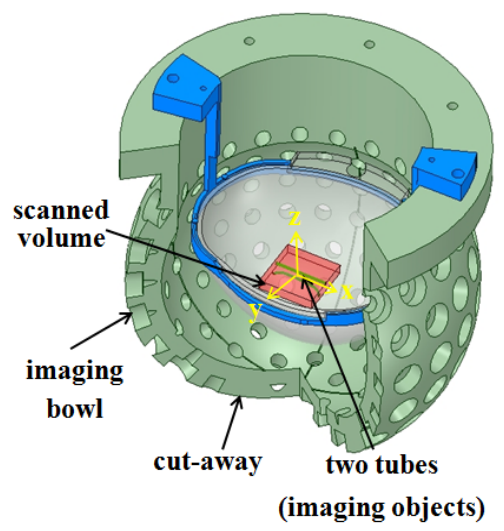

Figure 2.2. (a) Skull mold, (b) a 3D CAD model of the skull phantom, (c) a skull phantom which has a transcranial thickness of $1.52 \mathrm{~mm}$, a length of $79.3 \mathrm{~mm}$, width of $63 \mathrm{~mm}$ (not including flanges), and a height of $31.5 \mathrm{~mm}$, and (d) a 3D CAD model 
of the transducer array with the skull in place during the calibration scan; tip of the fiber optic that is attached to the robot is the point source; the green lines represent the central lines of the scan at the middle $x y$ plane and the red and blue lines represent the grid locations at the opposite side of the $x$ axis and along the $x$ axis, close to the front and back edges of the skull; (e) a 3D CAD model of the positions of transducers 1, 2, and 3 in relation to the scan volume (red cuboid) and also location of three of the four fibers that illuminates center of the transducer array, (f) a 3D CAD model of the position of the two tubes (green cylinder) in the transducer array.

\subsubsection{Imaging process}

The Opotek laser was used to illuminate the tubes; it was tuned at a peak absorption wavelength of ICG (780nm light beam). Laser illumination was guided through the 4legged optical fiber bundles, which were connected to the top periphery of the transducer array. Figure 2.2 (e) indicates the locations of three of the fibers illuminating the center of the transducer array. By illuminating the tube, sets of the PA data were recorded by the PAI system and formed the $\boldsymbol{g}$ matrix (given in equation (2.1)). Then, a threshold was applied to eliminate the low amplitude noise from the recorded PA signals. The ratio between the applied threshold and maximum amplitude of the PA signals in the presence of the skull was 0.078 and in the absence of the skull was 0.29 . Since the skull attenuated the PA signals, the threshold applied to the signals obtained through the skull was about one fourth of that in the absence of the skull.

As explained in section 1.3.1, to acquire the image of the object, $f$, the $g$ matrix should be multiplied by the regularized pseudo-inverse of the imaging operator $\left(\boldsymbol{H}_{\boldsymbol{k}}^{+}\right)$. The $\boldsymbol{H}_{\boldsymbol{k}}^{+}$was 
computed in Matlab before the imaging experiment. In order to regularize the pseudoinverse of the imaging operator $90 \%$ of the smallest singular values were replaced by zeros.

Finally, pixel values of the object-image were obtained by computing the multiplication of $\boldsymbol{g}$ and $\boldsymbol{H}_{\boldsymbol{k}}^{+}$matrices. The image display was rendered offline with a 3D slicer after applying an image threshold of $4.5 \%$.

\subsection{Results}

\subsubsection{Skull Phantom}

In our earlier work, the acoustic properties of different materials were measured, and based on the result, a mixture of epoxy and titanium dioxide powder was selected for the skull-mimicking material [11]. Epoxy samples with different concentrations of titanium dioxide powder and different thicknesses were made to investigate a closest match to neonatal skull bone in terms of acoustic properties. Figure 2.3(a) shows the acoustic insertion loss of three samples with different thicknesses $(1.1 \mathrm{~mm}, 2.2 \mathrm{~mm}$, and $4.2 \mathrm{~mm}$ ), each composed of 50\% epoxy and 50\% titanium dioxide powder, as well as the acoustic insertion loss of the infant skull bone. Acoustic insertion loss of neonatal skull bone (red curve) was derived from the literature (see Ref [8]). Figure 2.3 (b) shows the acoustic insertion loss as a function of the frequency of four cured epoxy samples with different concentration of titanium dioxide powder $(14 \%, 16 \%, 43 \%, 50 \%)$, and the same thickness $(\sim 1.4 \mathrm{~mm})$ in comparison to the neonatal skull bone. 
(a)

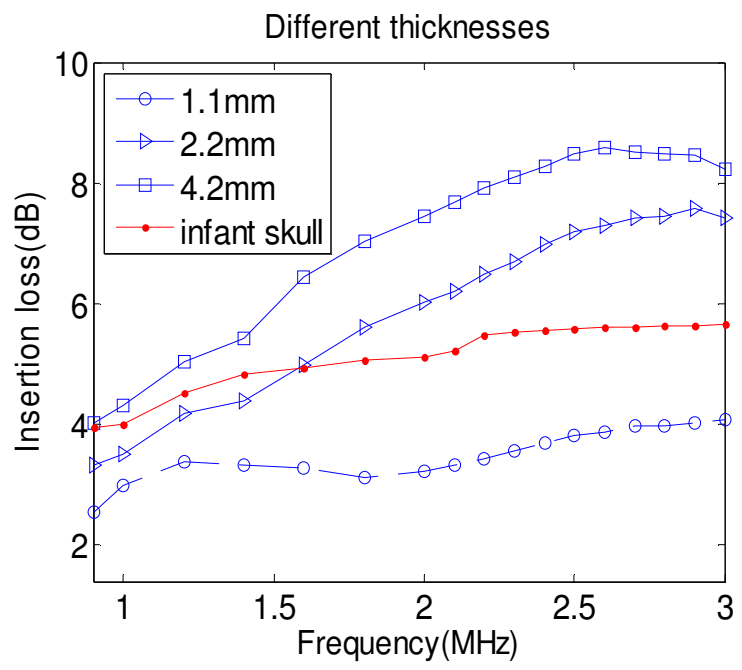

(b)

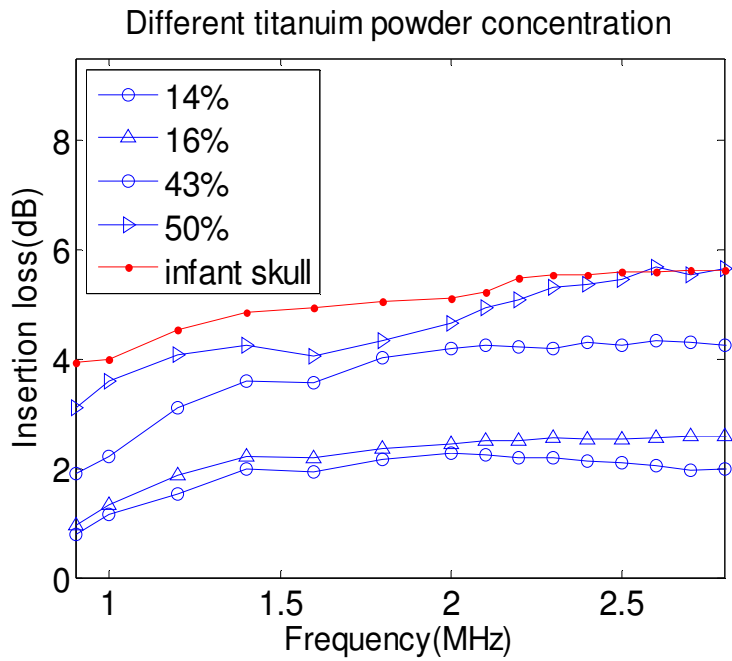

Figure 2.3. Acoustic insertion loss of different samples prepared from mixtures of epoxy and titanium dioxide powder. (a) Acoustic insertion loss of the samples composed of $50 \%$ epoxy and $50 \%$ titanium dioxide powder with different thicknesses compared to acoustic insertion loss of the skull (adapted from Ref $[28,43]),(b)$ Acoustic insertion loss of samples with the same thickness $(\sim 1.4 \mathrm{~mm})$ and different proportions of titanium dioxide powder compared to acoustic insertion loss of neonatal skull.

The ultrasound insertion loss of the skull and all of the samples increased with frequency. As the proportion of titanium dioxide powder and the thickness of the samples were increased, the acoustic insertion loss increased. The acoustic insertion loss of the sample with $50 \%$ titanium dioxide powder and 50\% epoxy was closest to the acoustic insertion loss of neonatal skull bone, across all frequencies tested (Figure 2.3 (b)). Based on these measurements, a 1.4-mm thick sample composed of 50\% (by mass) titanium dioxide powder and $50 \%$ epoxy was predicted to be the most similar to neonatal skull bone. 
We utilized 3D prototyping techniques to scan the head of a realistic infant doll and design a mold; we then fabricated the mold from solid Teflon®. Using the mold, a skull phantom with a heterogeneous thickness was successfully fabricated. The average thicknesses of the skull at its front, center, and back side were approximately $1.63 \mathrm{~mm}$, $1.42 \mathrm{~mm}$, and $1.53 \mathrm{~mm}$, respectively. Because this skull phantom was thicker than the targeted skull phantom, lower concentration of titanium dioxide powder (44\%) was chosen to have almost the same acoustic properties as neonatal skull.

\subsubsection{Imaging operator}

Figure 2.4 shows representative subsets of the imaging operator with and without the skull phantom for three different locations: $\boldsymbol{x}$ : opposite side of the $\boldsymbol{x}$ axis (close to the front of the skull), $\boldsymbol{x}$ : along the $\boldsymbol{x}$ axis (close to the back of the skull), and center of the skull phantom. Each of the three mentioned locations cover four scan lines which are shown in Figure $2.2(\mathrm{~d})$ ). The $\boldsymbol{x}$ coordinate for the $\boldsymbol{- x}$, center, and $\boldsymbol{x}$ lines varies respectively from $-9.75 \mathrm{~mm}$ to $-8.25 \mathrm{~mm},-0.75 \mathrm{~mm}$ to $0.75 \mathrm{~mm}$, and $8.25 \mathrm{~mm}$ to 9.75 $\mathrm{mm}$. The $\boldsymbol{y}$ coordinate for the lines of the three areas ranges from $-9.75 \mathrm{~mm}$ to $9.75 \mathrm{~mm}$, and the $z$ coordinate remains constant (since all of these lines are on the central $x \boldsymbol{y}$ plane $(z=3.5 \mathrm{~mm})$.

Each panel of Figure 2.4 consisted of temporal signal responses of three transducers at 160 grid locations. To better understand the transducer responses to the scan, responses from transducers at three different locations were chosen for further study. As shown in Figure 2.2 (e), transducer 1 was on the opposite side of the $x$ axis and opposite side of the $\boldsymbol{y}$ axis $(-\boldsymbol{x}-\boldsymbol{y})$ of the scan volume; transducer 2 was on the opposite side of transducer 1 (along the $\boldsymbol{x}$ axis and the $\boldsymbol{y}$ axis $\boldsymbol{x y}$ ) and on the same rung as transducer 1; and transducer 
3 was close to the bottom of the imaging cavity and close to the center of the scan volume $(\boldsymbol{x}=0, \boldsymbol{y}=0)$.

Each row of each panel of Figure 2.4 shows data from three transducers at four scan lines over 160 grid locations. The section of the imaging operator corresponding to the first and second transducers resembled a $\mathrm{W}$-shaped wave, while the section corresponding to transducer 3 appears as an almost straight line. The result was expected as the time of arrival of each acoustic wave at each transducer was related to the distance between the transducer and the grid point. In this case, the distance between the grid points and the first/second transducer varied while the distance between the grid points and the third transducer was almost constant.

Additionally, transducer 1, at the opposite side of the $\boldsymbol{x}$ axis, was close to the $\boldsymbol{- x}$ area. Therefore, its $\mathrm{W}$-shaped response from the $\boldsymbol{- x}$ area (first panel of Figure 2.4 (a)) arrived earlier than the center (second panel of Figure 2.4 (a)), and its W-shaped response from the center arrived earlier than the $\boldsymbol{x}$ area (third panel of Figure 2.4 (a)). Conversely, the $\mathrm{W}$-shaped response of transducer 2 arrived earlier from the $\boldsymbol{x}$ area than the $\boldsymbol{x}$ area and the centre.

The two imaging operators, one with, and one without, the skull layer, were compared in order to determine the effect of the skull layer on the PA signal propagation, in terms of both amplitude loss and time delay. The photoacoustic signals in the imaging operator measured in the absence of the skull phantom presented curved patterns with higher amplitudes than that of the photoacoustic signals captured in the presence of the skull (Figure 2.4(a)). 
(a)

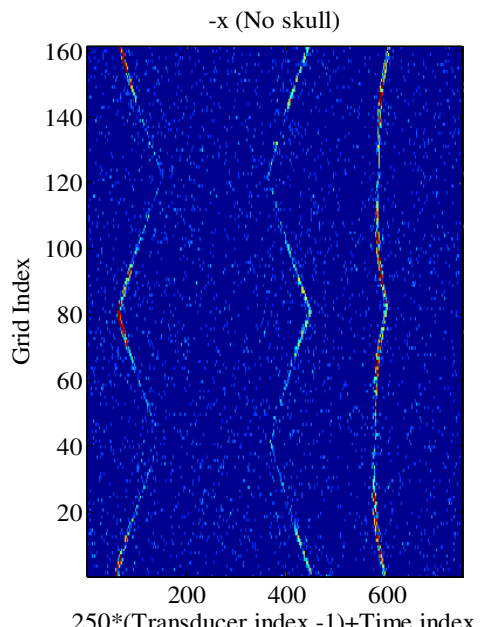

(b)

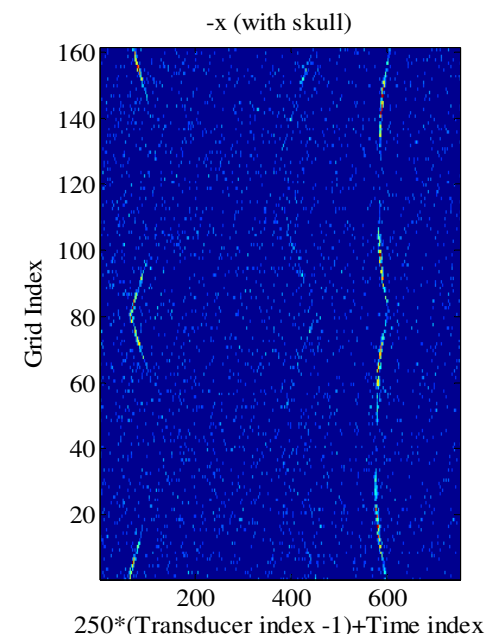

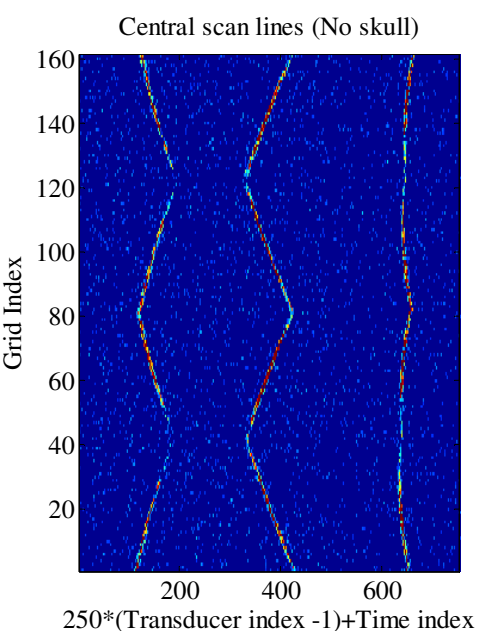
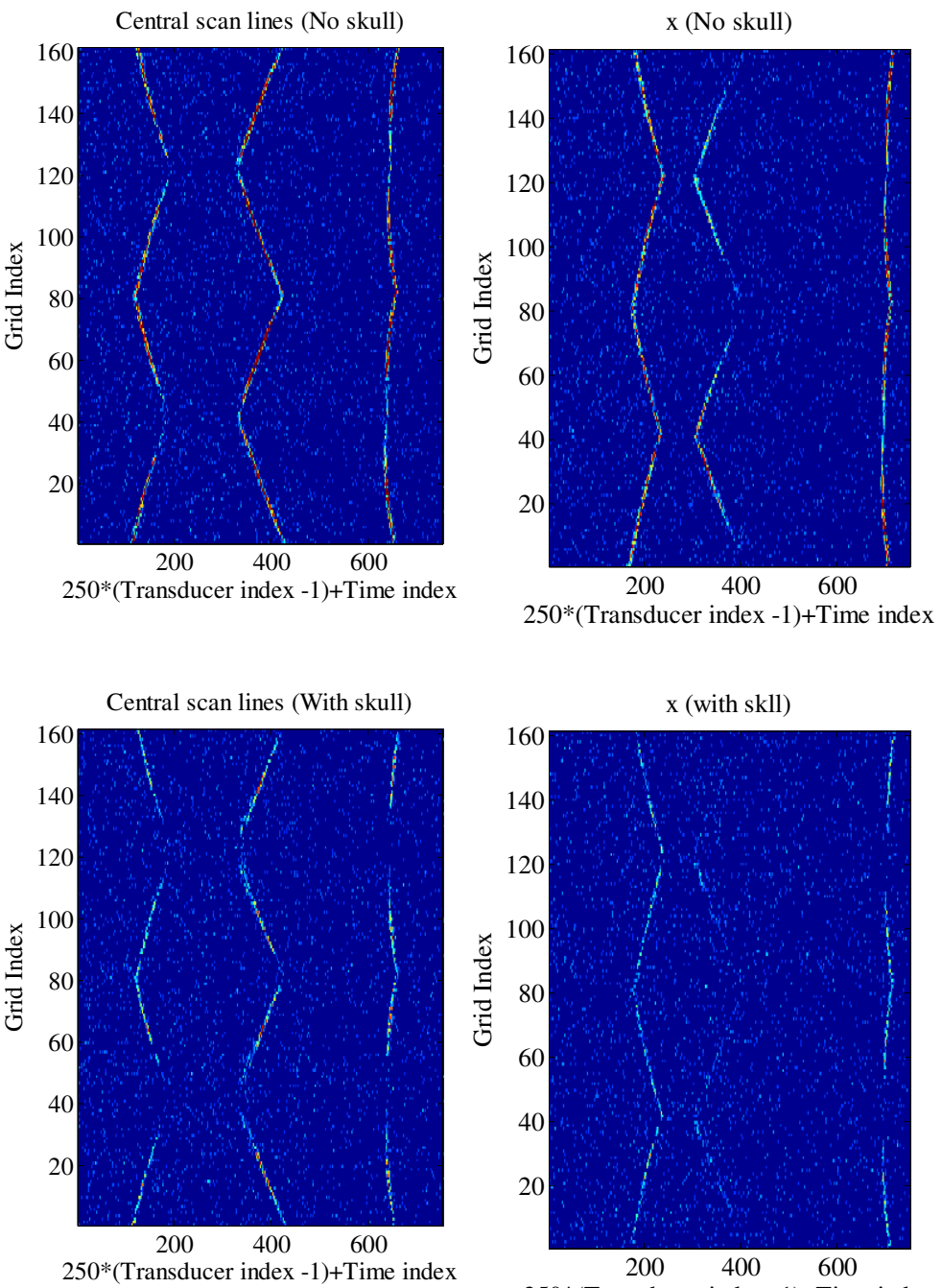

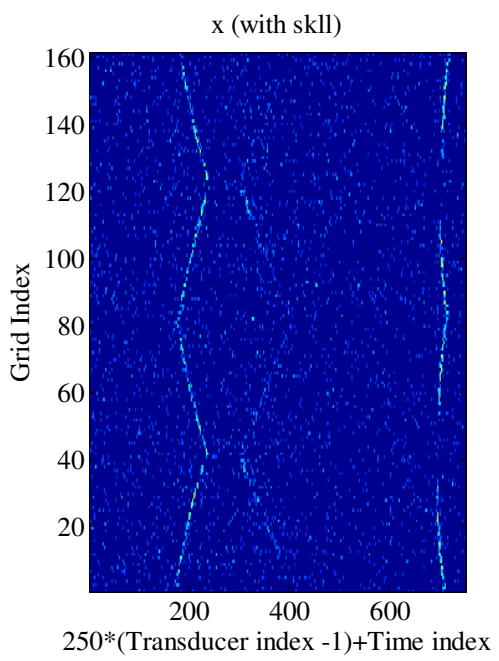

Figure 2.4. (a) three representative subsets of the imaging operator measured without the skull phantom at three different locations (the three transducers are shown in Figure 2.2 (e)): four scan lines being $\sim 9 \mathrm{~mm}$ from the center of the volume on the opposite side of the $x$ axis (four red lines in Figure 2.2 (d)); four central scan lines (four green lines in Figure $2.2(\mathrm{~d})$ ); four scan lines being $\sim 9 \mathrm{~mm}$ from the center of the imaging along the $x$ axis (four blue lines in Figure 2.2 (d)); (b) three subsets of the imaging operator measured with the skull phantom, at the same locations and the same transducers as (a). Each row of each subset shows data from 
transducer one, two, and three (Figure 2.2 (e)) at 160 grid locations. Only 250 time points are displayed for each transducer signal due to data preprocessing procedures that involved subset selection and down-sampling of the data.

When the skull phantom was in place, however, the corresponding photoacoustic signal patterns became weaker, shifted in time, broken in places, and were sometimes followed closely by a weak noisy pattern (Figure 2.4 (b)). The imaging operators at the $\boldsymbol{- x}$ area, center, and $\boldsymbol{x}$ area were also compared to determine the sensitivity of the system to different areas. Our results showed that the system was most sensitive in the detection of PA signals generated at the center of the imaging cavity, less so at the $-\boldsymbol{x}$ area, and least sensitive at the $\boldsymbol{x}$ area.

The trigger time of the laser was monitored by examining the time of arrival of the PA signal at the monitoring transducer. The PA signals measured on the monitoring transducer were expected to arrive at the same delay time relative to the laser pulse regardless of the grid location due to the fixed orientation and distance from the PA point source. However, small differences were observed at different grid locations within each calibration scan, and also between calibration scans. For example, when the skull phantom was present the delay was $0.9 \pm 0.04 \mu$ s, while during the scan without the skull phantom the delay was $0.85 \pm 0.03 \mu \mathrm{s}$. The difference in the maximum delay time represented fewer than 4 samples on the DAQ running at $40 \mathrm{MHz}$ and was likely due to a small difference in water temperature between the two calibration scans. The variation in delay time relative to the mean delay time was $2.2 \%$ for the scan without the skull and $3.1 \%$ for the scan with the skull. During the lengthy scans the water temperature in the imaging cavity increased due to power dissipation of the transducer preamplifiers. The 
raise in temperature increased the speed of sound in the water and decreased the time of arrival of the PA signal on the monitoring transducer (see Figure 2 (b) \& (c)).

The variations in the laser pulse energy were estimated from the maximum amplitude of the photoacoustic signal recorded on the monitoring transducer. The coefficient of variation $(\mathrm{CV})$ was computed using the photoacoustic signal amplitude measured at all grid locations, and was 16.5 for the calibration scan with the skull phantom, and was 15.8 for the calibration scan without the skull phantom. Six large signals were recorded during the scan with the skull phantom and four spikes during the scan in absence of the skull phantom. Overall, the laser pulse energy changes were considered small or rare in occurrence and corrections to the imaging operators were not performed.

\subsubsection{Photoacoustic imaging of different objects}

Figure 2.5 presents photoacoustic images of one tube, and two tubes, filled with various concentrations of ICG $(500 \mu \mathrm{M}, 250 \mu \mathrm{M}, 100 \mu \mathrm{M}, 50 \mu \mathrm{M}$, and $1.25 \mu \mathrm{M})$ captured in the presence, and then the absence, of the skull phantom. The contrast of the images was diminished when the ICG concentration was decreased. The photoacoustic images collected in the absence of the skull phantom were observed to have higher contrast than those collected in the presence of the skull phantom.

In addition, the system could detect images of one tube filled with the minimum concentration of $1.25 \mu \mathrm{M}$ ICG in the absence of the skull, while the one tube filled with $50 \mu \mathrm{M}$ ICG was barely captured through the skull, as seen in the last image of Figure 2.5 (b). The system could still reconstruct images of two tubes filled with $1.25 \mu \mathrm{M}$ ICG; however, the system with the skull could not reconstruct both tubes successfully, even in 
the $50 \mu \mathrm{M}$ ICG case, where half of the right tube was not detected (as shown in Figure 2.5(d)). This last result was likely a consequence of the difference in sensitivity of the system to different areas of the imaging cavity.

(a)

$500 \mu \mathrm{M}$ $250 \mu \mathrm{M}$

$100 \mu \mathrm{M}$

$50 \mu \mathrm{M}$

$1.25 \mu \mathrm{M}$

No skull
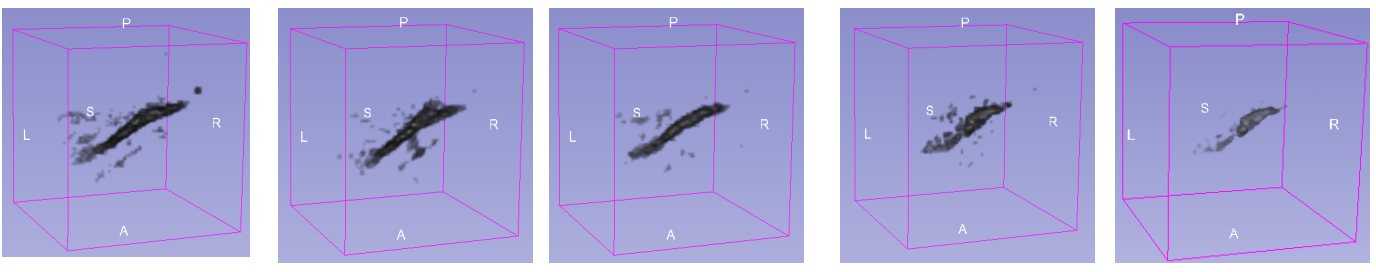

(b)
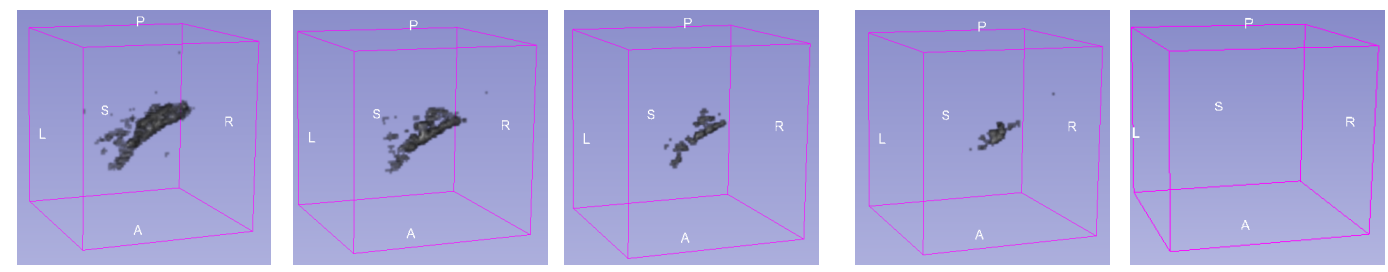

skull

(c)
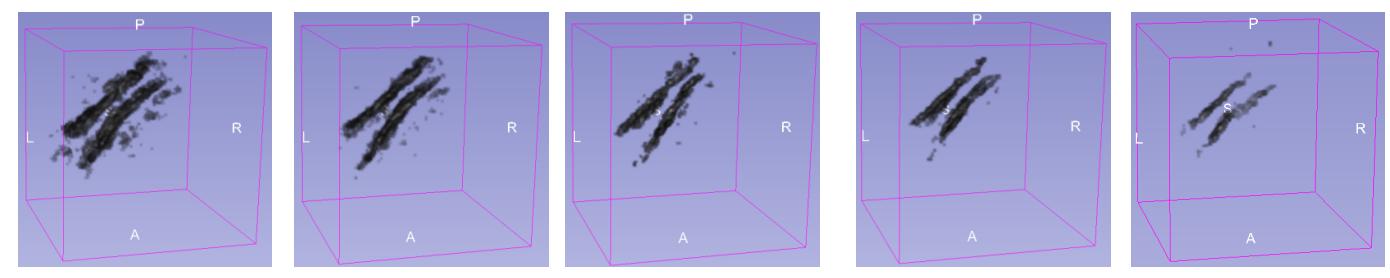

(d)
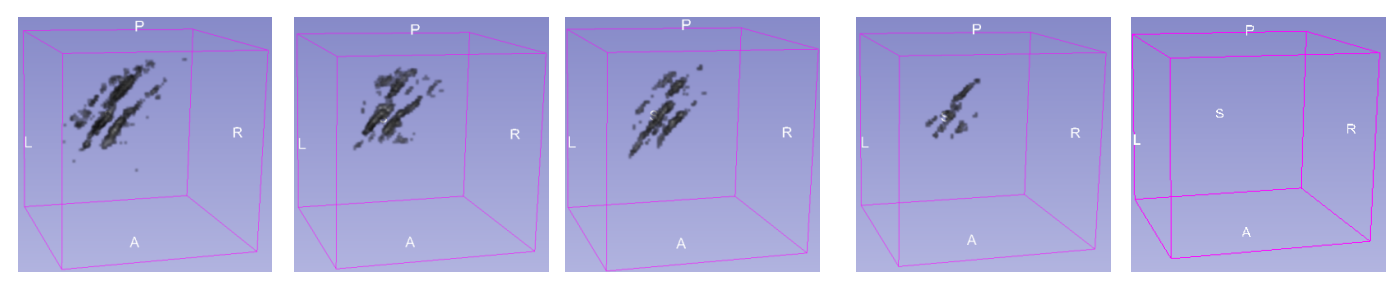

skull

Figure 2.5. Photoacoustic images of one tube, and two tubes, filled with different concentrations of ICG $(500 \mu \mathrm{M}, 250 \mu \mathrm{M}, 100 \mu \mathrm{M}, 50$, and $1.25 \mu \mathrm{M})$, and captured in the absence and the presence of the skull phantom. The threshold applied for images of the tubes with $1.25 \mu \mathrm{M}$ ICG was half of the threshold applied for other images. 
With the skull phantom present, the corresponding images of the tubes were closely followed by a weak noise pattern. In the image of one tube filled with $500-\mu \mathrm{M}$ ICG captured through the skull (Figure 2.5(b)), the noise pattern likely resulted in spurious pixels nearby the tube and this has made the tube appear thicker.

\subsection{Discussion}

The PAI system was capable of imaging ICG-filled tubes through the skull phantom. The lowest detectable ICG concentration through the skull phantom was $50 \mu \mathrm{M}, 40$ times higher than that of the PAI system with no skull phantom. The average insertion loss of the skull phantom at central transducer and central grid locations was estimated to be 4.5 $\mathrm{dB}$, which means about $33 \%$ of the PA signal energy was transmitted through the skull phantom. For concentrations lower than $50-\mu \mathrm{M}$ ICG, the PAI system was not able to reconstruct images of the tubes from the attenuated PA signals that were propagated through the skull.

The PAI system presented different sensitivity values within the multidimensional system at different areas of the transducer array. Since the system has a higher sensitivity to the $-\boldsymbol{x}$ area than the $\boldsymbol{x}$ area, half of the tubes in the $-\boldsymbol{x}$ area were better detected than the other half. For example, in the last image of the two parallel tubes in Figure 2.5 (d) half of the right tube was captured, while the other half was not detected. Additionally, in all images, more noise was apparent in the $\boldsymbol{x}$ area than the $-\boldsymbol{x}$ area.

By comparing subsets of the imaging operators- one with, and one without, the skull layer, or in different areas of the imaging volume, the effects of the skull layer on PA signal propagation, in terms of amplitude loss and time delay, were determined. In the 
presence of the skull phantom, the photoacoustic signal patterns became weaker, broken in places, and were sometimes followed closely by a weak noisy pattern (Figure 2.4). The weakness of the PA signal and the broken areas were a result of either amplitude attenuation caused by the skull, or acoustic reflection at the surface of the skull. Moreover, there was a shift in time of arrival of the PA signal; this was a result of the difference in speed of the sound in the skull phantom vs. in water. The speed of sound was approximately $2.35 \mathrm{~mm} / \mu \mathrm{s}$ in the skull phantom and $1.50 \mathrm{~mm} / \mu \mathrm{s}$ in water. Therefore, in the presence of the 1.52 -mm-thick skull, it was expected that the PA signal would arrive approximately $0.37 \mu$ s sooner than in the absence of the skull, which was close to the experimental result of $0.32 \mu \mathrm{s}$.

The skull-mimicking material was chosen based on matching the acoustic properties of the phantom and that of a neonatal skull, and after experimenting with several composite materials and measuring their acoustic properties. The properties of different mixtures of epoxy and titanium dioxide powder were measured and compared with the acoustic properties of neonatal skull bone. According to our measurements, when the proportion of titanium dioxide powder was increased, the acoustic insertion loss increased. Additionally, the results revealed an overall increase in acoustic insertion loss as the thickness of the sample was increased. Furthermore, the insertion loss was strongly dependent on the test frequency, with higher frequencies resulting in higher acoustic insertion loss. Based on these measurements, a 1.4-mm-thick sample composed of 50\% (by mass) titanium dioxide powder and 50\% epoxy was predicted to be the most similar to neonatal skull bone. 
The skull phantom with an average thickness of $1.52 \mathrm{~mm}$ was fabricated from $44 \%$ titanium dioxide powder and 56\% epoxy. An average PA signal amplitude attenuation caused by the skull phantom was experimentally measured to be $4.45 \mathrm{~dB}$ for the central transducer (2.7 MHz central frequency and $127 \%$ bandwidth), looking at four central lines of the calibration scan. According to the literature, the ultrasound attenuation of neonatal skull bone changes from $4 \mathrm{~dB}$ to $6 \mathrm{~dB}$ for the frequency range of $1 \mathrm{MHz}$ to 4 $\mathrm{MHz}$, which was close to the ultrasound properties of the fabricated skull phantom $[8,9]$.

During the calibration scan, one transducer attached to the point source was used to monitor changes in laser pulse energy as well as changes in laser firing time. The variation of arrival times of the PA signal to the monitoring transducer were $3.6 \pm 0.11 \mu \mathrm{s}$ and $3.4 \pm 0.07 \mu$ s for scans with and without the skull, respectively. These small variations could be due to a water temperature increase and laser firing time changes during a scan process. There were $15.8 \%$ to $16.5 \%$ differences in laser energy, both with and without the skull cases, during the scan process.

The image of the tubes through the skull was not clear at the $50-\mu \mathrm{M}$ ICG concentration, and the system could not obtain reliable images of the tubes at lower concentrations. Although applying image enhancement may help in recovering PA signals through amplification, the skull layer may have an unrecoverable effect on PAI sensitivity.

Our focus has been on studying the effects of the skull on sound propagation. However, the skull attenuates light propagation as well. In designing the skull phantom, the neonatal fontanels have not been considered. These boneless spots can be used for optical 
illumination. However, a method has been proposed to transfer light into a human brain using a photon recycler [10].

In the past decade, PAI has been developed rapidly for various medical applications, including the detection of early-stage breast cancer and the detection of prostate cancer,

as well as the measurement of tissue properties and molecular content [14]. Although some groups are working on brain imaging applications with PAI, this research is still at an early stage. Recently, a group from Washington University acquired photoacoustic images of a canine brain through a human skull by increasing light transmittance through the skull [10]. Our work, however, focused on the ultrasound effects of the skull bone.

\subsection{Conclusion}

A mixture of epoxy and titanium dioxide powder was selected as a skull-mimicking material. A skull-mimicking phantom with an average thickness of $1.52 \mathrm{~mm}$ made of $56 \%$ epoxy and $44 \%$ titanium dioxide powder had a very close acoustic insertion loss to that of neonatal skull bone. The skull phantom was fabricated and used to study the effects of the skull on photoacoustic signal propagation. The skull phantom distorted the photoacoustic signal. Most of the distortions were in a linear regime in the form of signal attenuation and phase shifts. Calibration scans of the system with and without the skull were acquired and compared, and were used for image reconstruction. Photoacoustic images of vessel-like tubes filled with ICG were captured. We started imaged tubes with a $500-\mu \mathrm{M}$ ICG, and then decreased the concentration of ICG to $1.25 \mu \mathrm{M}$. The photoacoustic imaging system was able to capture images of tubes containing $1.25-\mu \mathrm{M}$ ICG; however, with the presence of the skull, images could only be reconstructed of tubes filled with $\geq 50-\mu \mathrm{M}$ ICG. 


\section{References}

1. J. J. Volpe, "Neurobiology of periventricular leukomalacia in the premature infant," Pediatr. Res. 50, 553-562 (2001).

2. J. J. Volpe, "Cerebral white matter injury of the premature infant-more common than you think," Pediatrics 112, 176-180 (2003).

3. J. J. Volpe, "Encephalopathy of prematurity includes neuronal abnormalities," Pediatrics 116, 221-225 (2005).

4. B. Y. Huang and M. Castillo, "Hypoxic-ischemic brain injury: imaging findings from birth to adulthood," Radiographics 28, 417-39; quiz 617 (2008).

5. X. Wang, Y. Pang, G. Ku, X. Xie, G. Stoica, and L. Wang, "Noninvasive laser-induced photoacoustic tomography for structural and functional in vivo imaging of the brain," Nat. Biotechnol. 21, 803-806 (2003).

6. M. Li, J. Oh, X. Xie, G. Ku, W. Wang, C. Li, G. Lungu, G. Stoica, and L. V. Wang, "Simultaneous molecular and hypoxia imaging of brain tumors in vivo using spectroscopic photoacoustic tomography," Proc IEEE 96, 481-489 (2008).

7. X. Yang and L. V. Wang, "Monkey brain cortex imaging by photoacoustic tomography," J. Biomed. Opt. 13, 044009 (2008).

8. X. Wang, D. L. Chamberland, and G. Xi, "Noninvasive reflection mode photoacoustic imaging through infant skull toward imaging of neonatal brains," J. Neurosci. Methods 168, 412-421 (2008).

9. X. Wang, J. B. Fowlkes, D. L. Chamberland, G. Xi, and P. L. Carson, "Reflection mode photoacoustic imaging through infant skull toward noninvasive imaging of neonatal brains," Proc. SPIE, Photons Plus Ultrasound 7177, 717709 (2009). 
10. L. Nie, X. Cai, K. Maslov, A. Garcia-Uribe, M. A. Anastasio, and L. V. Wang, "Photoacoustic tomography through a whole adult human skull with a photon recycler," J. Biomed. Opt. 17, 110506 (2012).

11. P. Tavakolian, R. Todd, I. Kosik, A. Chamson-Reig, F. Vasefi, K. S. Lawrence, and J. J. L. Carson, "Development of a neonatal skull phantom for photoacoustic imaging," Proc. SPIE. 8581, Photons Plus Ultrasound 8581, 858146 (2013).

12. P. Tavakolian, I. Kosik, A. R. Chamson, K. S. Lawrence, and J. J. L. Carson, "Potential for photoacoustic imaging of the neonatal brain," Proc. SPIE, Photons Plus Ultrasound 8581, 858147 (2013).

13. M. B. Roumeliotis, R. Z. Stodilka, M. A. Anastasio, E. Ng, and J. J. Carson, "Singular value decomposition analysis of a photoacoustic imaging system and 3D imaging at 0.7 FPS," Opt. Express 19, 13405-13417 (2011).

14. L. V. Wang and S. Hu, "Photoacoustic Tomography: In Vivo Imaging from Organelles to Organs," Science 335, 1458-1462 (2012). 


\section{Chapter 3}

\section{D photoacoustic imaging through the skull phantom with phase and amplitude correction}

\subsection{Overview}

This chapter focuses on the processing and enhancement of the 3D PA images of objects acquired with the skull in place. The chapter applies a correlation coefficient metric in order to study the effects of the skull on PA signals detected at different voxels (grid locations) of the imaging volume. Then, it describes the methods developed for image enhancement and explains the correction of 3D PA images of a optically-absorbing rod that was captured with the skull phantom.

As mentioned in chapters 1 and 2, to reconstruct a PA image of an object, a calibration scan of the PAI system is needed. A new calibration scan of the PA system for a larger volume size was captured; it will also be explained in this chapter.

\subsection{Introduction}

Periventricular leukomalacia (PVL) is a major form of white matter brain injury in premature infants [1,2]. Ultrasonography is the most easily accessible technique for neonatal brain imaging. Although this modality is sensitive in detection of cystic PVL, it is relatively insensitive to diffuse PVL [1-4]. Photoacoustic imaging (PAI), which uses the merits of both light and ultrasound, should be more sensitive in detection of noncystic PVL.

In previous work, we fabricated a skull phantom which mimicked ultrasound properties of neonatal skull. Then, we investigated the effect that the skull layer has on the 
propagation of PA signals with the aim of PAI through a skull layer. Finally, we captured 3D images of tubes filled with indocyanine green (ICG) in the presence of the skull phantom, which demonstrated that PAI of vessel-like targets was possible through a layer with acoustic properties similar to the neonatal skull. Preliminary results of this work have been recently presented $[5,6]$.

In this work, our objective was to correct the effect that the skull layer has on the propagation of PA signals with the aim of PA image correction. We measured the signals generated from a PA point source at a multitude of grid locations to obtain imaging operators for the same transducer array explained in chapter 2. The imaging operators were used to compute cross-correlation, time delay and amplitude-ratio maps representative of the effect of the skull phantom on PA signal propagation. Then, the time delay and amplitude-ratio maps were utilized to develop a skull-correction method. The normalized cross-correlation map was used to study the distortion caused by the skull on different locations on the imaging volume. Finally, we tested the method on captured images of a light-absorbing rod through the skull phantom. Our results demonstrated that the skull effects were measurable and correctable.

\subsection{Methodology}

The PA system utilized to capture the PA signals of a optically-absorbing rod is the same as that introduced in section 2.3.1. Furthermore, the method utilized to reconstruct PA images from the captured PA signals was explained in section 2.3.2 above. 


\subsubsection{Imaging experiment}

\subsubsection{Imaging operator}

A new calibration scan of the imaging system was captured, which included 20 planes perpendicular to the $z$ axis and 40 by 40 grid points within each plane in the $\boldsymbol{x}$ and $\boldsymbol{y}$ directions. For acoustic coupling, the transducer array was filled with water. The scan was captured with a step size of $0.5 \mathrm{~mm}$. The grid was located near the center of the array. At each of the grid locations, the point source, with a diameter of about $0.4 \mathrm{~mm}$, was illuminated by a $1064 \mathrm{~nm}$ laser beam. Then, photoacoustic signals were recorded for approximately $25 \mu$ s from each of the 127 transducers simultaneously. The point source was scanned across each $\boldsymbol{x}$-coordinate from the lowest grid point to the highest, and then the $\boldsymbol{y}$-coordinate was incremented once; this process was then repeated. After scanning a square of $20 \mathrm{~mm} \times 20 \mathrm{~mm}$, the $z$-coordinate was incremented once, until the robot scanned a rectangular cuboid volume of $20 \mathrm{~mm} \times 20 \mathrm{~mm} \times 10 \mathrm{~mm}$. This scan covered a larger volume than the previous scan explained in chapter 2 . The scan volume was half of the potential imaging volume of the PAI system. The skull phantom was positioned within the hemispherical imaging cavity. The imaging cavity and the phantom were filled with water for acoustic coupling. The same procedure was repeated with the skull in the imaging cavity, and another imaging operator was captured in the presence of the skull.

Each calibration scan took about three hours to complete and resulted in a data set of approximately 8 GB. For each calibration scan, the main user interface of the SonixDAQ was set to 1000 sample numbers, $50 \mu$ s line duration, $2 \mu$ s gain delay, $0 \mathrm{mV}$ gain offset, 6 $\mu$ s receive delay, with a digital DC filter, fixed TGC and minimum TGC curve, 8GB 
buffer size, and $40 \mathrm{MHz}$ sampling rate. The data saved on the memory of the DAQ was downloaded on the workstation that was connected to the DAQ by the USB.

The imaging operators were processed to eliminate noise and to reduce the reconstruction time. Although the signal from each transducer included 1000 sample points, each signal was down-sampled 4 times to 250 points during the preprocessing procedures. The PA signals were rectified to provide non-negative signals. Then, a threshold was applied for both imaging operators (captured with and without the skull) in order to eliminate low amplitude noise. The ratio between the applied threshold and the maximum amplitude of the PA signals for the scan with the skull was 0.0176 , and 0.0148 for the scan without the skull.

\subsubsection{Imaging process}

An optically-absorbing rod was glued to a fiber which was attached to the arm of the robot (Figure 3.7Figure 3.7). Then, the rod was positioned at the center of the imaging volume. The imaging process explained in section 2.3.3.4 was followed in order to capture images of the rod. Some details, however, were changed. First, a Surelite laser was used to illuminate the rod with $1064 \mathrm{~nm}$ laser light. Second, a different value of the threshold was applied to eliminate the low amplitude noise from the recorded PA signals of the rod. The ratios between the applied threshold and maximum amplitude of the PA signals were 0.0104 in the absence of the skull, and 0.0146 in the presence of the skull. Third, the image display was rendered offline with 3D-Slicer after applying an image threshold of $31 \%$. Signal to noise ratio (SNR) of each image was computed in MATLAB. To measure SNR, the PA signals emitted by the rod were separated from the background 
noise. Then, the SNR was obtained by calculating the square of the amplitude ratio of signal to the background noise.

The images captured in the presence and absence of the skull were then quantitatively analyzed. On each image, length and diameter of the rod was computed. To measure the size of the rod, first, a 2D image of the rod was obtained by averaging all 20 planes along the $z$ axis. This time the image display was rendered offline with MATLAB without applying any image threshold. Then, two lines that were perpendicular to the rod were selected and the intensity profiles along these lines were measured and averaged. The size

of the rod was estimated from the width at the $1 / \sqrt{2}$ of the line intensity profile.

\subsubsection{Photoacoustic signal enhancement method}

As mentioned earlier, distortions caused by the skull resulted in signal attenuation and phase shifting. This section describes the studies performed to examine the distortions in the PA signals that propagated through the skull. Then, it describes the method we utilized to reconstruct enhanced PA images. Finally, this section provides detailed analyses of the distortions caused by the skull phantom on the calibrated scanned volume.

\subsubsection{Computing the effects of the skull on PA signals}

The correction method compares the two PA signals captured in the presence and the absence of the skull received at one transducer, and computed several metrics (Figure 3.1). The method computed the difference between the arrival times and the ratio of the maximum amplitude. For example, in Figure 3.1, the difference between arrival time of the peak of the PA signal captured in the presence of the skull compared to that of the PA 
signal captured in the absence of the skull is $-0.23 \mu \mathrm{s}$. In addition, the ratio between the maximum amplitudes of the signals captured with and without the skull is 0.61 .

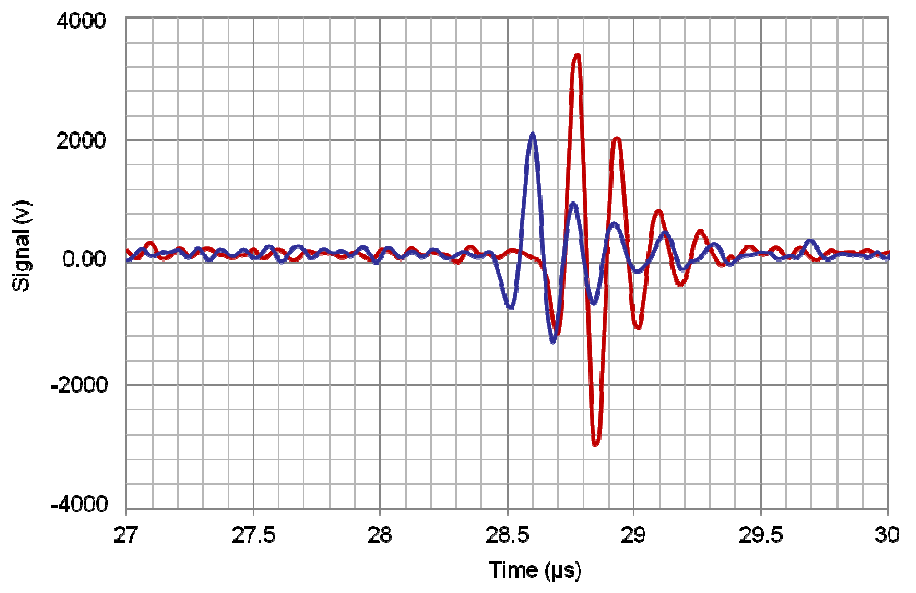

Figure 3.1. Photoacoustic signal propagation through a 1.4-mm thick skull phantom composed of $50 \%$ epoxy and $50 \%$ titanium dioxide powder. The red curve represents the PA signal without the skull in place and the blue curve represents the PA signal on the same transducer after passing through the skull layer. The PA point source was located near the center of the array.

To measure the difference between arrival times of the peak of the two PA signals, a cross-correlation operator was used. Considering the PA signal propagated through the skull and water as , and the PA signal propagated through only water as, the cross-correlation of the two signals ( ) can be obtained as follow: 
The difference between the arrival times of the peak of the two PA signals can be computed from the cross-correlation of the two signals. As shown in Figure 3.2 when the peak of $f[n]$ arrives at A in time trace and the peak of the PA signal captured through the skull $(g[n])$ arrives at $\mathrm{B}$, then the peak of $h[n]$ will occur at $C$ :

$$
\begin{gathered}
{[D, C]=\max (h[n]) ;} \\
C=\text { Number of time points }-A+B
\end{gathered}
$$

where $D$ is the maximum amplitude of the cross-correlation and $C$ is the place it occurs in time trace. Therefore, we can measure the shift in arrival time from $C$ by the following equation:

$$
\text { Delay shift }=B-A=C-\text { Number of points }
$$

The amplitude attenuation of the skull was measured directly by computing the ratio between the maximum amplitude of the PA signal captured in the presence of the skull over the maximum amplitude of the PA waveform captured in the absence of the skull.

$$
\text { Scaling ratio }=\frac{\max (g[n])}{\max (f[n])}
$$

For each of the 127 transducers in the imaging cavity, the comparison of the PA signals was performed for all grid points of the calibration scan. Then, the two criteria (amplitude attenuation and phase shift) were measured, resulting in two matrices: the 'Delay shift' map and the 'Scaling ratio' map. In the Results section below, the 'Delay shift' map and the 'Scaling ratio' map for three transducers are presented with further explanation. 
(a)

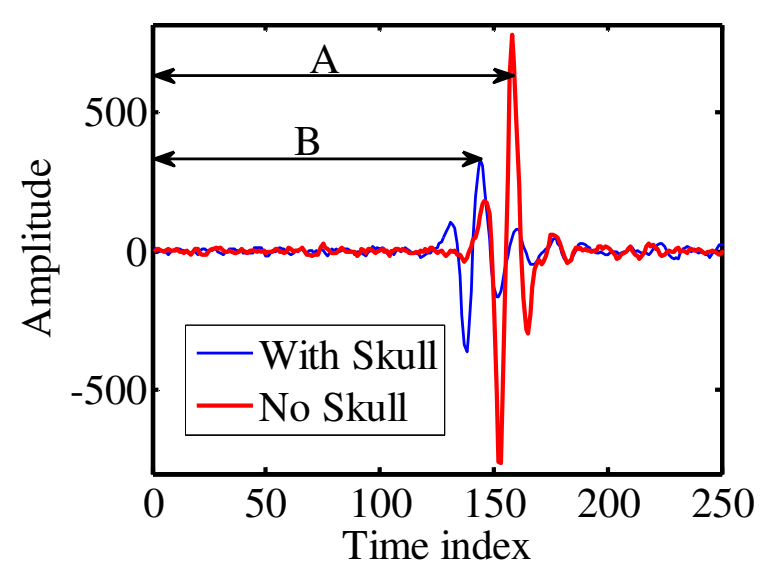

(b)

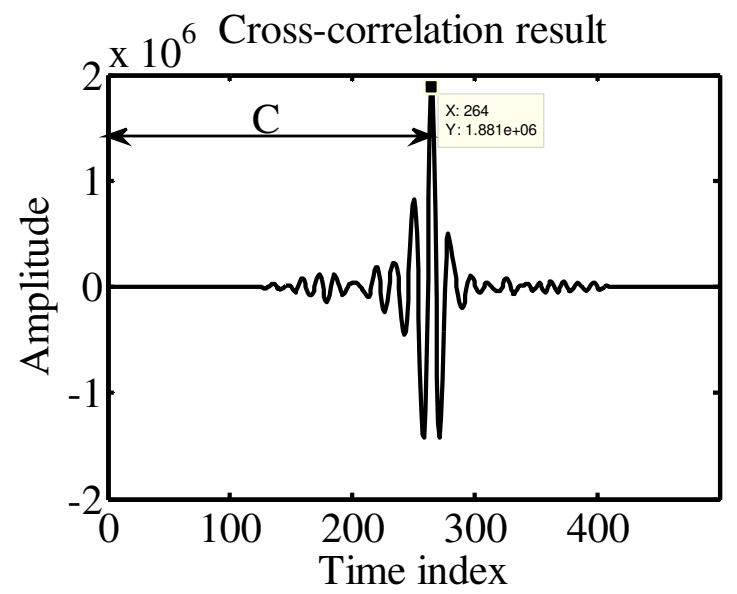

Figure 3.2. (a) Red curve: example of a PA signal captured in the absence of the skull at a transducer and one grid location. Peak of this signal is located at $\mathrm{A}$ in time trace. (a) Blue curve: the PA signal captured through the skull at the same transducer and the same voxel as red curve. Peak of this PA signal is located at B in time trace. (b) This waveform displays the result of cross-correlation of the two PA signal displayed in panel (a). The peak of this signal occurs at $C(C=250$ - $(A-B))$ in time trace.

\subsubsection{Skull correction method}

The correction method affected the imaging operator captured in the absence of the skull, which resulted in a corrected version of the imaging operator. To accomplish this result, each PA signal captured at each transducer for each grid location was corrected with respect to amplitude and phase shift distortion. First, the PA signal measured in the absence of the skull was shifted based on the computed difference of the time of arrival (equation (3.3) and using the circshift operator in MATLAB (equation (3.5). Then, this 
PA signal was attenuated by multiplying that to the corresponding scaling ratio of the two PA signals (3.6):

$$
\begin{aligned}
& f^{\prime}[n]=\operatorname{circshift}\left(f[n], \text { Delay }_{\text {shift }}\right) \\
& f^{\prime \prime}[n]=\text { scaling ratio } * f^{\prime}[n]
\end{aligned}
$$

This process resulted in a skull-corrected PA signal $\left(f^{\prime \prime}[n]\right)$, which was captured at one transducer and one grid location. To obtain the skull-corrected imaging operator, the procedure was repeated for all transducers at all grid locations. The computed imaging operator predicted the effects of the skull in terms of amplitude attenuation, and shift in arrival time of the PA signals.

\subsubsection{Analysis of the effects of the skull}

Cross-correlation of two signals provides a measure of similarity between two signals. We used cross-correlation to assess the effects the skull layer caused, by comparing the PA signals captured in the absence of the skull to those captured in the presence of the

skull. With this method, we were able to study the spatial-dependence of the effects of the skull by assessing the values of cross-correlation on different voxels. Crosscorrelation of the two PA waveforms, one captured through the skull and one in the absence of the skull, was computed for each of the 127 transducers and each of the grid points. Then, the maximum of the cross-correlation of the PA signals was measured. For each grid point, the sum of the maximum cross-correlation from each transducer was computed across all 127 transducers. Since the sensitivity of the system was spatially dependent, the values of the cross-correlation depended on an additional factor: the sensitivity of the system to the corresponding grid point in the imaging volume. 
In order to isolate the effect of the skull, we normalized the cross correlation. First, at each grid point and each transducer, the auto-correlation of a PA signal, either captured through the skull or in the absence of the skull, was measured. Using the same method as that for cross-correlation, the maximum amplitude of auto-correlation was computed. Afterwards, at each grid point, the maximum values were summed across all 127 transducers. Then, utilizing the following equation, the cross-correlation was normalized:

$$
\text { Normalized } x \text { corr }=\frac{(\max h[n])^{2}}{\max \left(\text { autocorr }_{N S}\right) * \max \left(\text { autocorr }_{W S}\right)}
$$

where autocorr stands for auto-correlation of the signal, captured either in the absence of the skull, NS (no skull) or through the skull, WS (with skull).

\subsection{Results}

\subsubsection{Effects of the skull on PA signals}

\subsubsection{Cross-correlation map}

The normalized cross-correlation values estimated the effects of the skull on separate voxels that have different positions in relation to the skull. When the value of the normalized cross-correlation was close to 1, the two PA signals captured through the skull and in the absence of the skull were identical. This implied that the PA signal propagating through the skull experiences no distortion. However, when the value was close to 0 , the two signals were dissimilar, thereby indicating that the PA signal is completely distorted by the skull. For intermediate values of the normalized crosscorrelation, the skull results in partial distortion of the PA signal. Figure 3.3 shows the normalized cross-correlation for all transducers at three different planes. The first plane is 
closest to the skull (Figure 3.3 (a)), and the last plane is farthest from the skull (Figure 3.3 (c)).

(a)

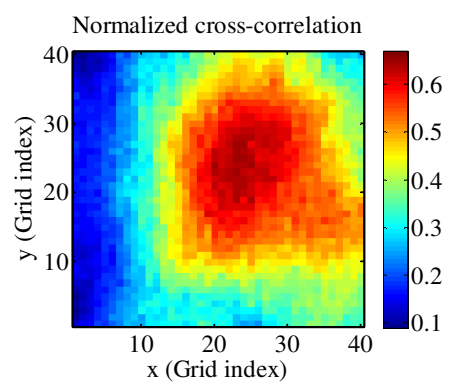

(b)

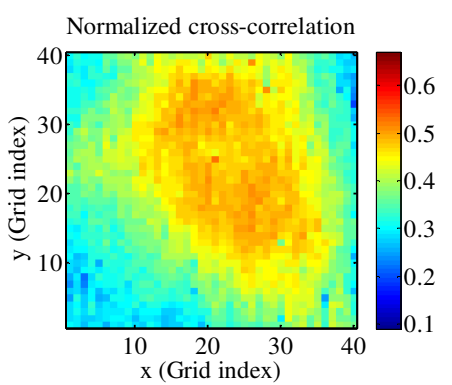

(c)

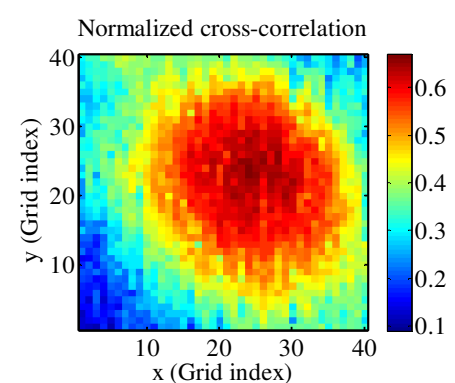

Figure 3.3. Normalized cross-correlation between PA signals captured through the skull and in the absence of the skull at all 127 transducers. In each panel, the value shows the normalized cross-correlation at each grid point of the imaging operator within a plane. Three $x y$ planes $(z=0 \mathrm{~mm}, z=5 \mathrm{~mm}, z=10 \mathrm{~mm})$ are displayed in the figure; (a) the first plane (closest to the surface of the skull), (b) the middle plane (central plane), and (c) the last plane (furthest from the surface of the skull). These axes are from 0 to 40 steps; therefore, based on the step size of the scan which is 0.5 $\mathrm{mm}$, each panel covers a square of $20 \mathrm{~mm} \times 20 \mathrm{~mm}$.

\subsubsection{Scaling ratio and Delay shift maps}

To compare and discuss the results of the measured 'scaling ratio' map and the 'delayshift' map, three transducers were chosen at three different locations around the transducer cavity. Positions of the selected transducers in relation to the imaging volume are identified in Figure 3.4 Error! Reference source not found.(a). 
The scaling ratio maps for transducers 1, 2, and 3 are presented in Figure 3.4 (b). Each value on the 'scaling ratio' map shows the ratio between the maximum amplitude of the two PA signals captured in the presence, and the absence, of the skull at one grid point. For each transducer, the 'scaling ratio' map is displayed for the first $\boldsymbol{x y}$ plane $(z=0 \mathrm{~mm}$, closest to the skull), the middle $x y$ plane $(z=5 \mathrm{~mm})$, and the top $x y$ plane $(z=10 \mathrm{~mm}$ the furthest from the skull).

(a)

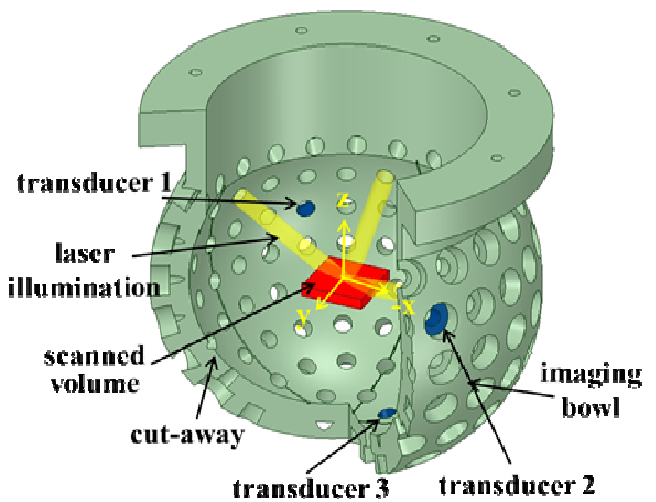

(b)

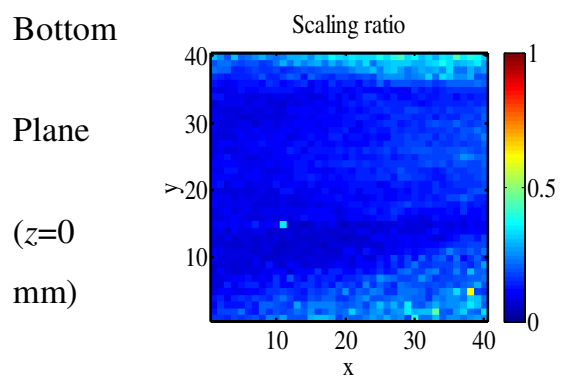

Central

Plane

$(z=5$

$\mathrm{mm})$

Transducer 1

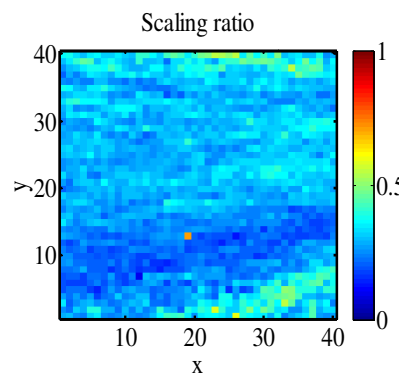

Transducer 2
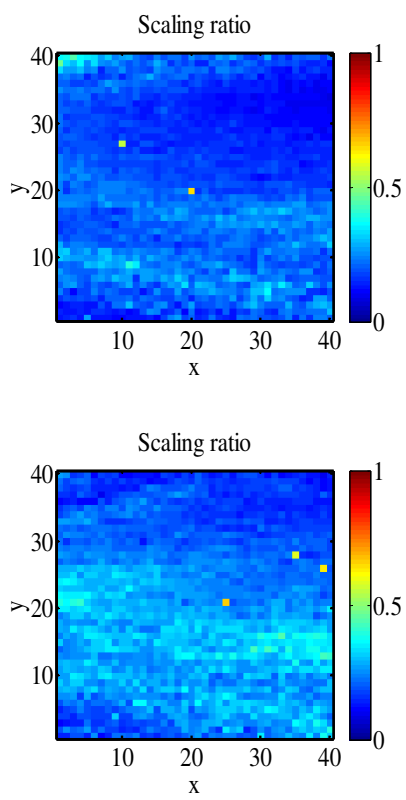

Transducer 3
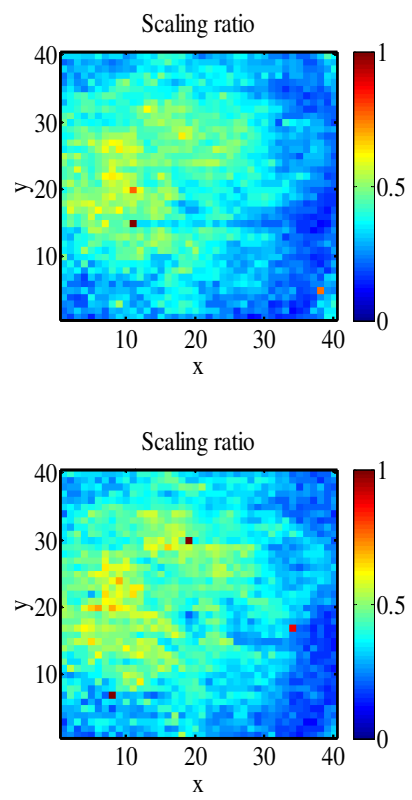

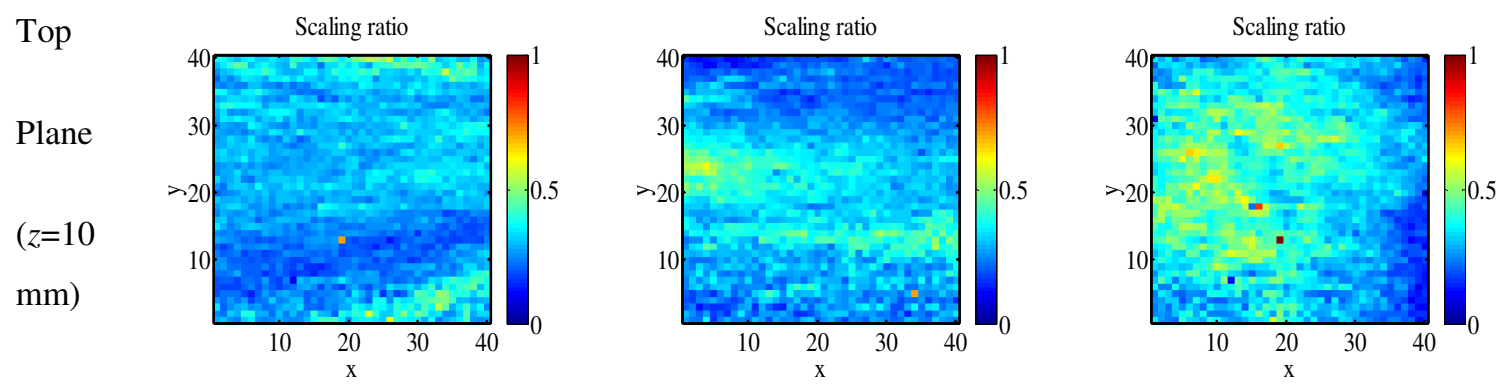

Figure 3.4. (a) PAI cavity showing positions of transducers 1, 2, and 3. (b) Scaling maps. Each value in each panel represents the amplitude attenuation of the PA signal due to the presence of the skull phantom at a specific voxel (grid location). In each panel the $x$ and $y$ axes are equal to the $x$ and $y$ axes shown inFigure 3.4. The axes increase in index from 0 to 40 . As mentioned above, each panel covers a square of $20 \mathrm{~mm} \times 20 \mathrm{~mm}$, based on the step size of the scan which was $0.5 \mathrm{~mm}$.

The 'Delay shift' maps for transducers 1, 2, and 3 are presented in Figure 3.5. Each value on the 'Delay shift' map shows the difference in the arrival time of the PA signal captured in the presence of the skull, and that captured in the absence of the skull, at one grid point. For each transducer, the 'Delay shift' map is displayed for the first $x \boldsymbol{y}$ plane $(\boldsymbol{z}$ $=0 \mathrm{~mm}$, closest to the skull), the middle $\boldsymbol{x y}$ plane $(z=5 \mathrm{~mm})$, and the top $\boldsymbol{x y}$ plane $(z=10$ $\mathrm{mm}$, the furthest from the skull).

Transducer 1

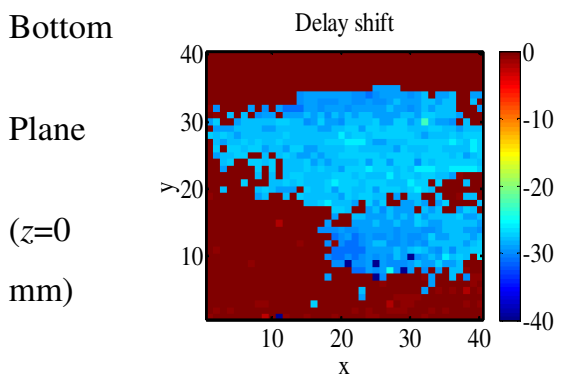

Transducer 2

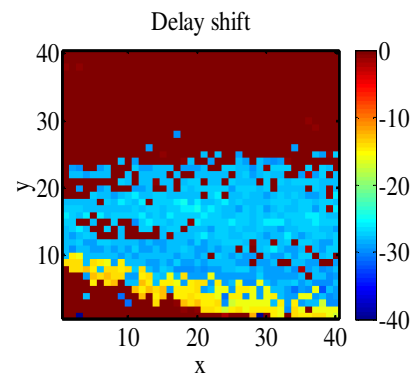

Transducer 3

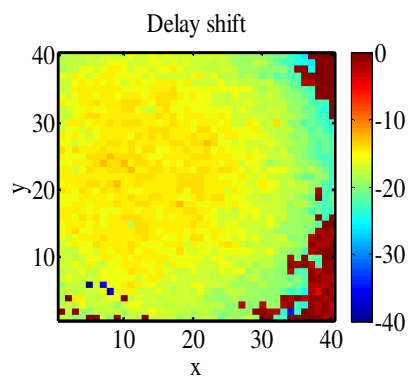



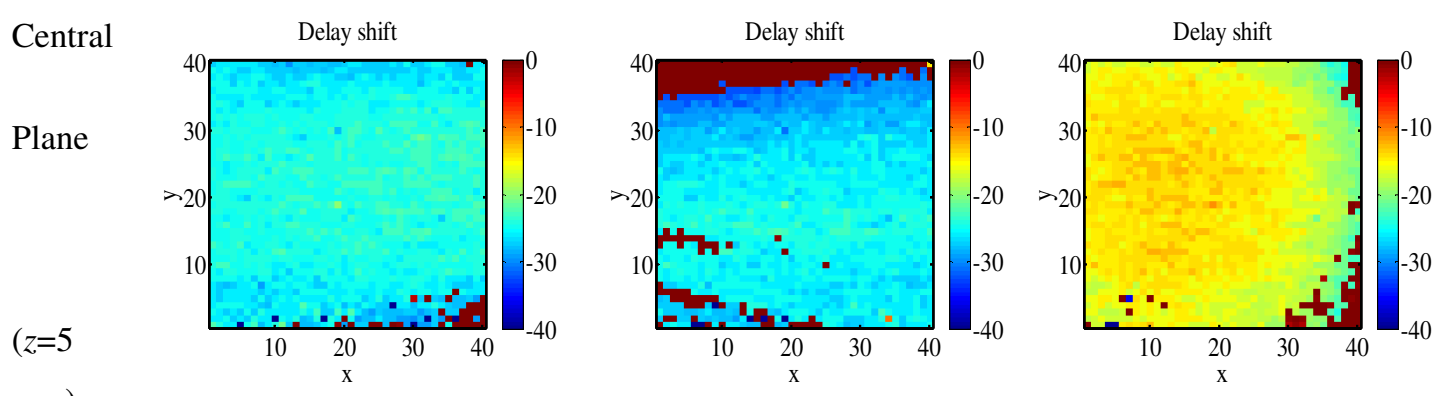

$\mathrm{mm})$

Top
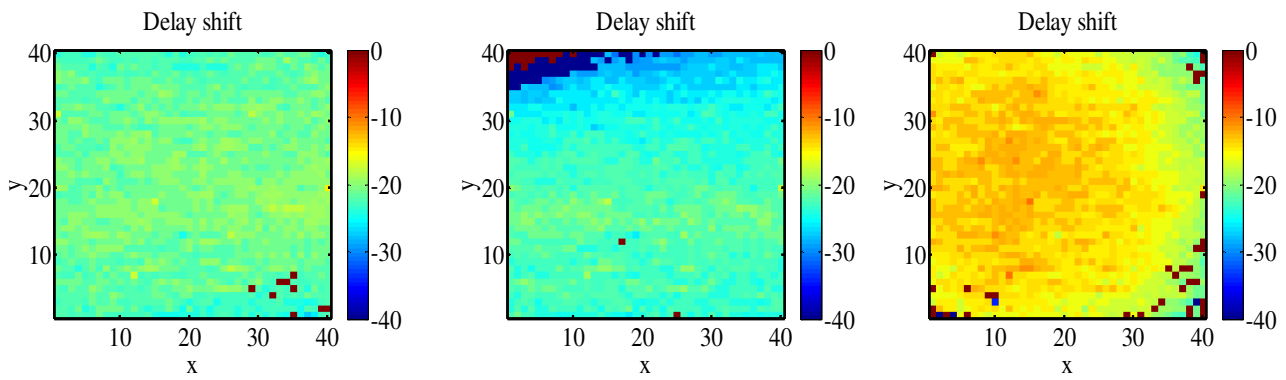

Figure 3.5. Delay maps. Each value in each panel shows the differences in time of arrival of the PA signal with and without the skull at a specific voxel (grid

location). In each panel the $x$ and $y$ axes are equal to the $x$ and $y$ axes shown in

Figure 3.4 (a).Figure 3.4. The axes are increasing from 0 to 40 steps. Each panel covers a square of $20 \mathrm{~mm} \times 20 \mathrm{~mm}$. Due to the PA sampling rate which was set to $40 \mathrm{MHz}$, each delay time point was 25 ns. Therefore, for all the panels, the delay values range from 0 to $1 \mu \mathrm{s}$.

Due to PA sampling rate which was set to $40 \mathrm{MHz}$, each delay time point was $25 \mathrm{~ns}$. Therefore, for all the panels, the delay values are distributed from $0(0 \times 25 \mathrm{~ns})$ to $1 \mu \mathrm{s}$ $(40 \times 25 \mathrm{~ns})$. The values were negative since the PA signal that propagated through the skull arrived earlier than the PA signal that propagated through the water only. Close to the surface the skull, the PA signal captured through the skull was significantly distorted. 
Therefore, because of the calculation problem, at these grid points and transducers, the delay shift value were represented as $0 \mathrm{~ns}$ in each panel.

\subsubsection{Corrected version of the imaging operator}

The original imaging operator, which was captured in the absence of the skull, was altered in order to account for the two effects of the skull. These effects were the change in the arrival time of the PA signal and the alteration in the amplitude of the PA signal. To obtain the new version of the imaging operator, each PA signal was corrected in terms of phase distortion based on its corresponding value on the 'Delay shift' map. Then, the PA signal was attenuated based on the value on the 'Scaling ratio' map. Error! Reference source not found.Figure 3.6 (a) and (d) present two subsets of the imaging operator captured in the absence of the skull at center (middle $x \boldsymbol{y}$ plane $(z=5 \mathrm{~mm})$ ) and one edge of the imaging volume (first $x y$ plane $(z=0 \mathrm{~mm})$ ). Error! Reference source not found.Figure 3.6Error! Not a valid bookmark self-reference.Figure 3.5 (b) and (e) present the same subsets as (a) and (d) captured in the presence of the skull. The skullcorrected version of the imaging operator captured in the absence of the skull is displayed in Error! Reference source not found.Figure 3.6 (c) and (f)Error! Not a valid bookmark self-reference.Figure 3.5 .

In Error! Reference source not found.Figure 3.6, each panel shows the response of one transducer at four different scan lines. Each scan line included 40 voxels (grid locations). The PA signals were recorded for approximately $25 \mu \mathrm{s}$ on each transducer. After data preprocessing procedures (explained in section 3.3.1.1), the transducer signal had 250 time points. 
(a)

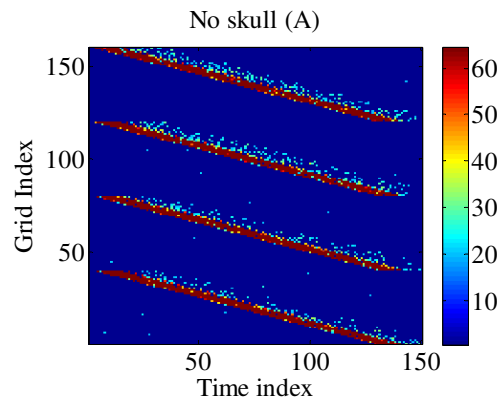

(d)

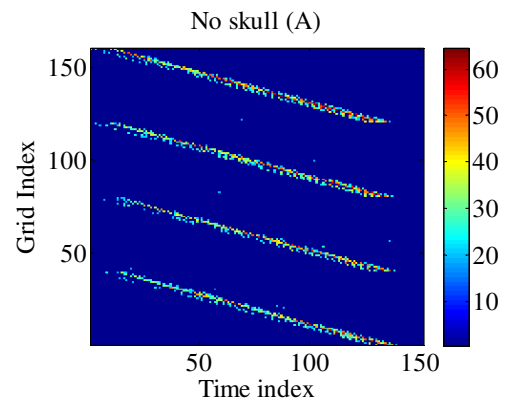

(b)

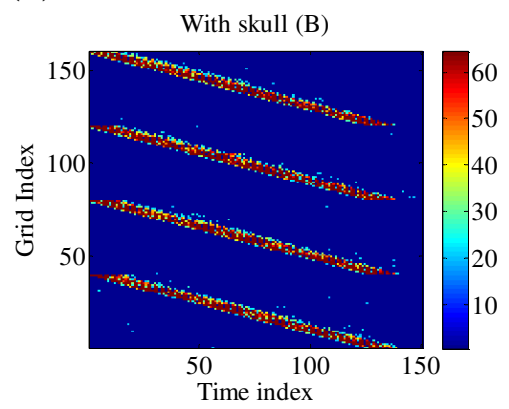

(e)

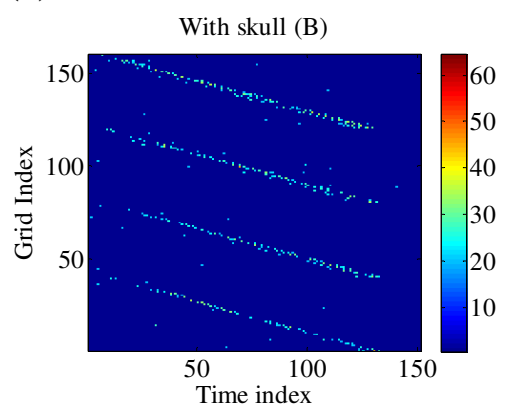

(c)

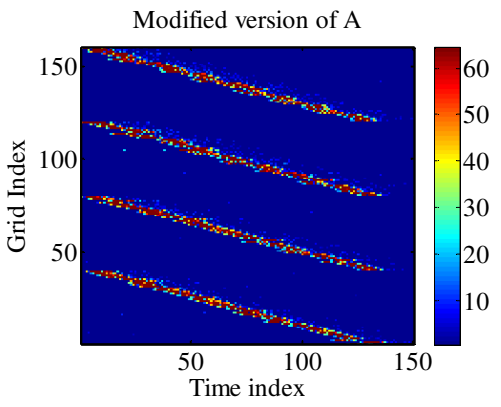

(f)

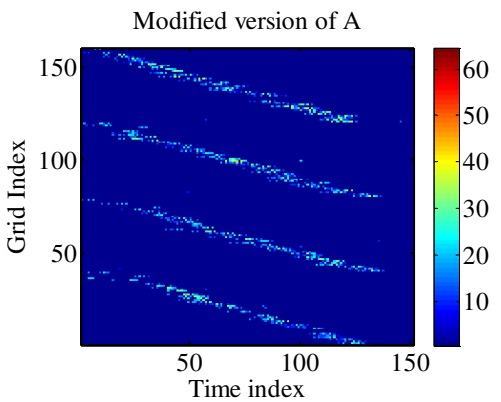

Figure 3.6. Demonstration of the data correction method. (a) Subset of the imaging operator measured in the absence of the skull phantom and at the center of the imaging volume $(z=5 \mathrm{~mm})$. (b) The same subset of the imaging operator measured in the presence of the skull. (c) The skull-corrected version of (a), which predicted the effects of the skull layer in terms of amplitude attenuation and shift in time of arrival. (d) Subset of the imaging operator measured in the absence of the skull phantom and at one edge of the imaging volume (the first $x y$ plane $(z=0 \mathrm{~mm}))$. (e) The same subset of the imaging operator as (d) measured in the presence of the skull. (f) The skull-corrected version of (d). Each row of each subset shows data from transducer one (Figure 3.4 (a)) at 160 central grid locations (4 central scan lines) of the imaging volume. Only 150 of possible 250 time points are displayed to enhance visualization. 
On each panel, only 150 time points are displayed in order to better visualize details of the imaging operator. The correction algorithm time-shifted the water-only PA signals earlier in time to account for the presence of the skull. For example, the time-shift effect can be seen by looking at the left end of each of the four scan lines. In the Error! Reference source not found.Figure 3.6 (a), the PA signals representative of the smallest $x$-values arrive between 5 to 10 time indices into each trace. However, for Error! Reference source not found.Figure 3.6 (b) and (c) the corresponding PA signals arrive closer to the zero time index. Furthermore, the PA signals captured in water alone (Error! Reference source not found.Figure 3.6 (d)) were higher in amplitude compared to the PA signals that propagated through the skull phantom (Error! Reference source not found.Figure $3.6(\mathrm{e})$ ). However, after the correction algorithm, the amplitudes of the PA signals in the skull-corrected imaging operator (Error! Reference source not found.Figure 3.6 (f)) were similar to the PA signals in the imaging operator captured through the skull (Error! Reference source not found.Figure 3.6 (e)).

\subsubsection{Image enhancement}

An imaging experiment was performed to demonstrate the skull-correction method. The computed skull-corrected imaging operator (IO) was used to reconstruct an enhanced image of an object using PA signals collected in the presence of the skull phantom. The

object used was a light-absorbing rod illuminated at $1064 \mathrm{~nm}$ (Figure 3.7 (a)). Sets of photoacoustic signals emitted from the rod were captured in the absence and presence of the skull phantom. The PA signals emitted from the rod were captured in the absence of the skull phantom and were reconstructed with the original IO. The captured 3D image of the rod is shown in Figure 3.7 (b).Then, PA signals of the rod, which were captured 
through the skull were reconstructed with the original IO (Figure 3.7 (c)), the IO captured in the presence of the skull ( Figure 3.7 (d)), and the corrected version of the original IO (Figure 3.7 (e)). The SNR of these images were calculated and presented in Table 3.1.

(a)

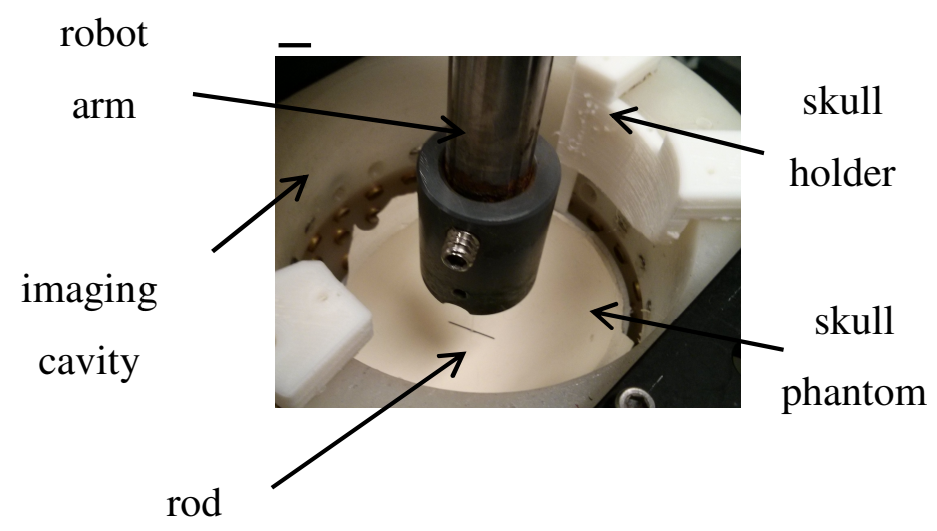

(b)

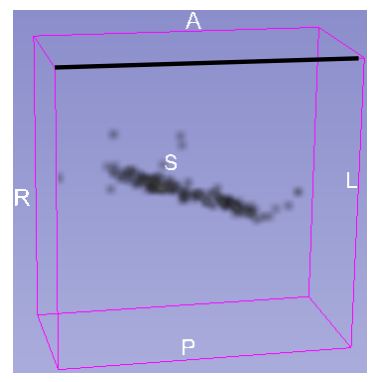

(c)

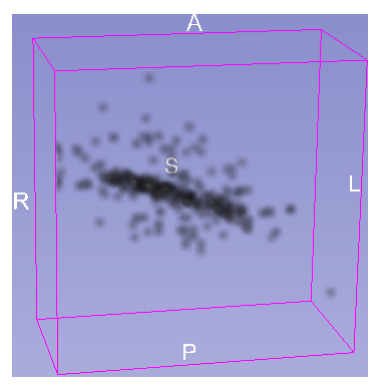

(d)
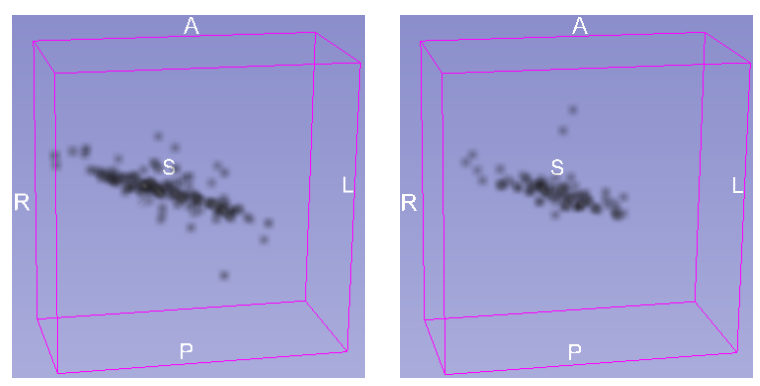

Figure 3.7. Position of a optically-absorbing rod with a diameter of $0.4 \mathrm{~mm}$ and length of $14 \mathrm{~mm}$ in relation to the skull and the imaging cavity. (b) A PA image of the rod captured in the absence of the skull and reconstructed with the original IO. (c) A PA image of the rod captured in the presence of the skull and reconstructed with the original IO. (d) A PA image of the rod captured in the presence of the skull 
and reconstructed with the IO captured in the presence of the skull. (e) A PA image of the rod captured in the presence of the skull and reconstructed with the skullcorrected IO.

Table 3.1. Signal to noise ratio of the images of the rod (shown in Figure 3.7)

\begin{tabular}{|l|l|l|l|l|}
\hline Image: & No Skull & With Skull & With Skull & With Skull \\
\hline Imaging operator & No Skull & No Skull & With Skull & Modified version of no skull \\
\hline SNR & 72.6 & 4.0 & 33.3 & 17.6 \\
\hline
\end{tabular}

Each PA image of the rod covered 20 images along the $z$ axes $(z=0 \mathrm{~mm}$ to $z=10 \mathrm{~mm})$. Intensity profiles of the images were averaged in order to obtain a $2 \mathrm{D}$ intensity image of the rod. An averaged intensity profile for the PA image of the rod captured in the absence of the skull and reconstructed with the original imaging operator is presented in Figure 3.8 (left panel). Intensity profiles along these lines were computed, averaged, and shown in Figure 3.8 (right panel).

The same method was utilized for the image of the rod that is captured with the skull and reconstructed with different imaging operators. Figure 3.8 (b) shows the result for the image reconstructed with original IO. Figure 3.8 (c) presents the result for the image reconstructed with IO that is captured through the skull (WS). Finally, the image that is reconstructed with skull-corrected IO is shown in Figure 3.8 (d).

In right column of Figure 3.8, the length of the rod was measured for all the captured PA images of the rod. For the first two images that are captured in the presence and absence 
of the skull and reconstructed with the original imaging operator, the length of the rod was estimated to be $11.2 \mathrm{~mm}$. The length of the rod in Figure 3.8 (c) was estimated to be $10.6 \mathrm{~mm}$, and $9.5 \mathrm{~mm}$ in Figure 3.8 (d).

(a)

Image: no skull
(NS)

Imaging operator (IO): NS
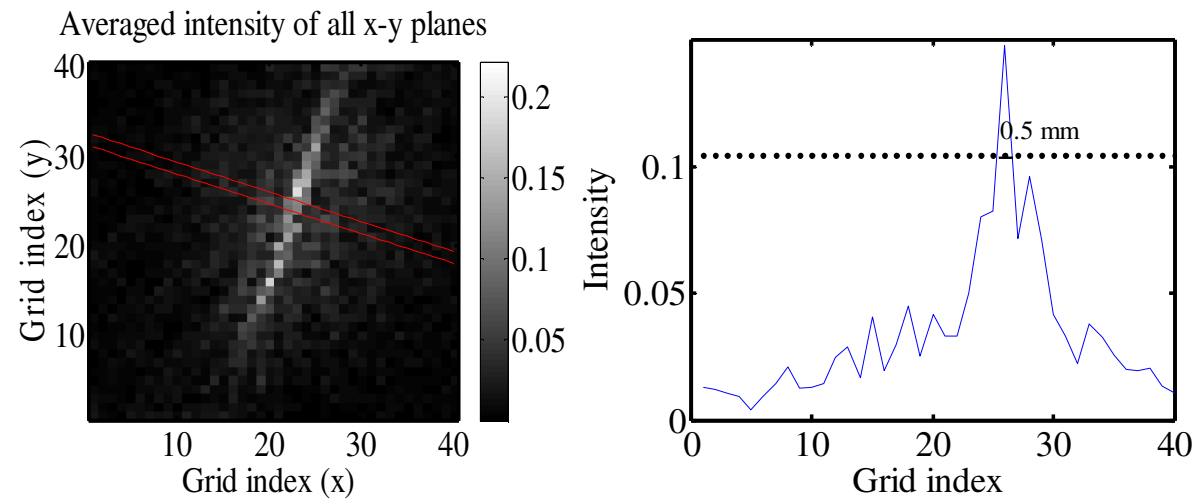

(b)

Image: with skull (WS)

IO: NS
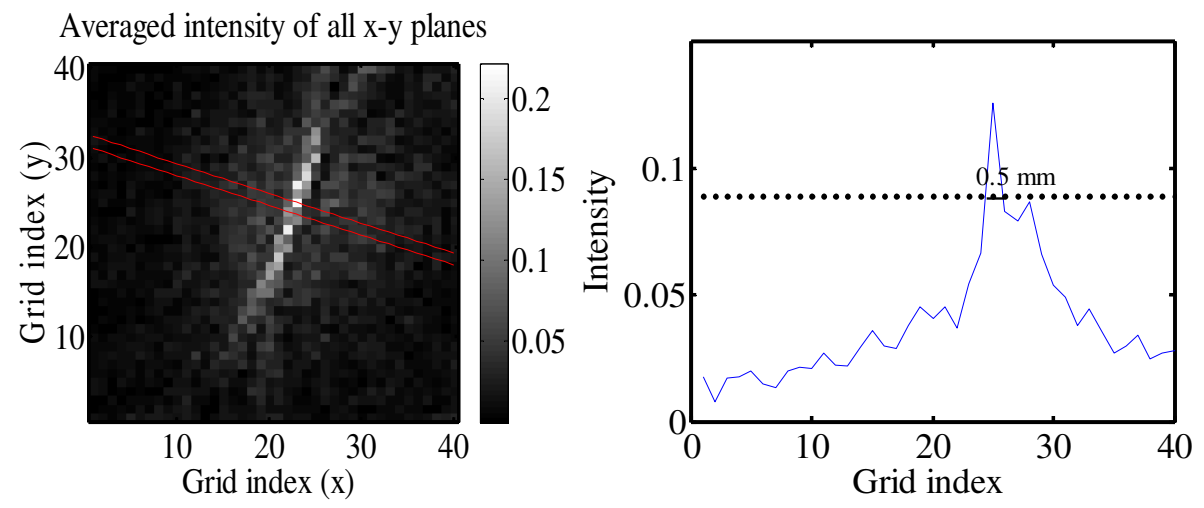

(c)

Image: WS

IO: WS
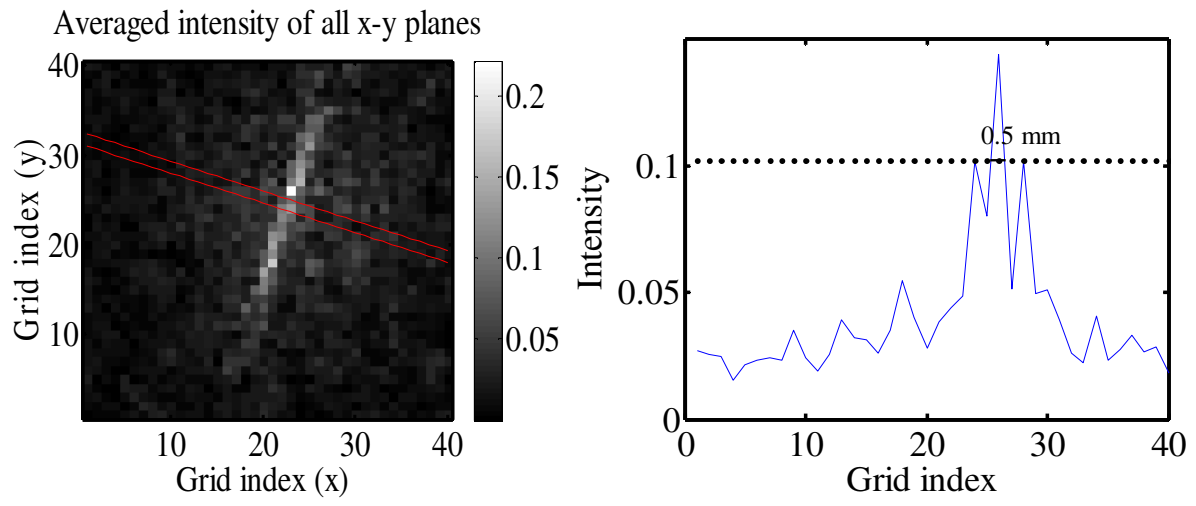
(d)

Image: WS

IO: skull-corrected

IO

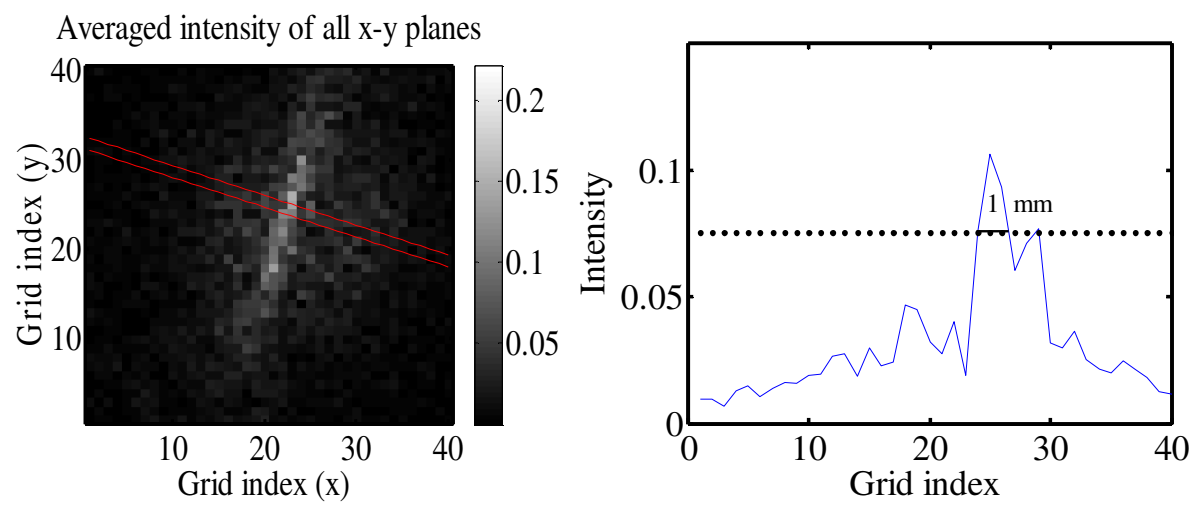

Figure 3.8. Averaged intensity along $20 x y$ planes of the rod (left column) and along a line normal to the rod (right column). The red lines on the panels in the left column display the location of the path used to extract the line profiles in the right column. On the panels in right column, averaged intensity profiles are displayed (blue line). On these diagrams, the width at the $1 / \sqrt{2}$ of the line intensity profile is displayed with a black dotted line. (a) The intensity profile for the image that was captured in the absence of the skull and reconstructed with the water-only IO. (b) The intensity profile for the image that was captured in the presence of the skull and reconstructed with the water-only IO. (c) The intensity profile for the image that was captured in the presence of the skull and reconstructed with the IO captured through the skull. (d) The intensity profile for the image that was captured in the presence of the skull and reconstructed with the skull-corrected IO.

In right column of Figure 3.8, the width at the $1 / \sqrt{2}$ of the line intensity profile are displayed. This factor is used to indicate the grid locations that have higher intensity compared to other voxels. Utilizing these diagrams, the diameter of the rod in all images 
was observed to be $0.5 \mathrm{~mm}$ except for the image reconstructed with the skull-corrected IO.

\subsection{Discussion}

We have developed a method to correct photoacoustic signals with respect to amplitude attenuation and arrival time distortions that result from propagation through a skull-like material. The method was investigated by capturing images of an optically-absorbing rod in the presence and absence of the skull. First, PA signals emitted from the rod were captured in both the presence and the absence of the skull. These signals were then reconstructed with three imaging operators: the original (water-only) IO, an IO that was captured through the skull, and a skull-corrected IO. Our skull-correction reconstruction method proved to be successful in reducing the noise from a 3D photoacoustic image of a light-absorbing rod. Use of this method was found to improve the photoacoustic signalto-noise ratio by 13.6 unit (see Table 3.1 ).

However, prior correction of the PA signals by applying the amplitude scaling and time delay shifts was unable to fully correct for the effects of the skull layer. The PA image of the rod that was captured through the skull and reconstructed with the original IO was observed to be $11.2 \mathrm{~mm}$ in length. However, the rod PA image that was captured through the skull and reconstructed with the skull-corrected IO was observed to be $9.5 \mathrm{~mm}$ in length. Since the true size of the rod was $14 \mathrm{~mm}$ in length and $0.4 \mathrm{~mm}$ in diameter, the skull-correction method was not fully successful. Additionally, the applied skullcorrection method resulted in reduced image resolution. Considering the intensity profile in Figure 3.8, the diameter of the rod that was captured through the skull and reconstructed with the skull-corrected IO was observed to be $1 \mathrm{~mm}$. 
In the future, this approach can be developed to precisely model the effect of the skull on PA signals, based on the characteristics of an infant skull (e.g. skull thickness). The model should also consider other effects of the skull such as multiple scattering in bone. Photoacoustic signals emitted from a point source and propagated through the skull have some ringing after the main PA signal, which is as a matter of this effect. The PA signals propagated through the skull can then be recovered from all these effects.

Although this study is the first research on experimentally-based phase-and-amplitude correction estimation in PA imaging, some methods have been proposed for correction of the skull distortions in high intensity focused US therapy. Adaptive correction method and phased-array correction modality were utilized to compensate the ultrasound distortion caused by the skull. Research on a fast algorithm of distortion correction on site is still ongoing [7].

A reconstruction methodology for transcranial PAT has been proposed that compensate for skull-induced aberrations of the recorded PA signals. In this reconstruction method, first the spatially varying speed of sound and mass density distributions of the to-beimaged subject's skull are determined by use of adjunct x-ray CT data. Then, the two criteria are employed with a time reversal image reconstruction method for the estimation of the initial amplitude of the PA signal within the brain [8]. Similarly, adaptive correction of the skull distortions in focused ultrasound uses some entry parameters, which are driven from a previously acquired 3D computational tomography (CT) scan of the skull bone structure [7]. Since there is a high cancer risk from radiation exposure to infants and children [9], these methods are not useful for infant's brain image correction from the distortions caused by the skull. Phased-array correction uses a multiple element, 
phased-array transducer driven by phase-and-amplitude controllable amplifier system to reconstruct ultrasound foci through the human skull [10].

The transmission of ultrasound waves through the skull bone(s) induces the conversion of longitudinal pressure mode to shear pressure mode. A recently-introduced reconstruction algorithm has been proposed that models and employs shear waves in PAT image formation. This study shows that when shear waves are omitted from the imaging model, a large number of spatial frequency components of the object will be mis-estimated $[11,12]$.

Similar to our modality, in the phase-array correction method, phase correction factors and amplitude correction coefficients were created by scanning an area of the plane normal to the acoustic propagation axis. A polyvinylidene fluoride (PVDF) needle hydrophone was used as an ultrasound receiver and spatially scanned by a steppingmotor-controlled three-dimensional positioning system. The skull was located between the phase-array transducer and the needle hydrophone. The scans were performed in the presence and absence of the skull for a $10 \mathrm{~mm}^{2}$ area of the focal plane at a scanning resolution of $0.2 \mathrm{~mm}$. At each point of the scan, the hydrophone response was recorded by a personal computer interfaced by a digital oscilloscope. The scan data gave the phase and amplitude distortion information that then was used for correction. When phase correction was implemented, the reconstructed single focus of acoustic intensity at the intended focal point resulted in an intensity increase. This method used an amplitude correction that homogenizes the acoustic intensity across a wavefront, which decreased the level of energy delivery at the focus. Therefore, when amplitude-and-phase correction 
was implemented the intensity of the reconstructed beam pattern was decreased compared to the time that only phase correction was implemented [10].

To study the effects of the skull on different locations of the imaging volume, we utilized a cross-correlation operator. The cross-correlation of the PA signals captured in the presence and the absence of the skull is a measure of the similarity of the two waveforms. Since the sensitivity of the PAI system is spatially dependent, the cross-correlation value also reflects the sensitivity of the system to their corresponding grid points of the imaging volume.

The cross-correlation was normalized with the measured auto-correlation of each of the PA signals, either captured through the skull, or captured in the absence of the skull. The normalization eliminated the effect of the amplitude of the PA signals, and consequently the effect of the sensitivity of the PAI system to the grid points of the imaging volume.

At the grid points that are located close to the surface of the skull, the normalized crosscorrelation values were very low, being distributed in the range from 0 to 0.2 . However, the normalized cross-correlation values were high for grid points that were far from the surface of the skull, which meant that the PA signals were more similar and less affected by distortion. For example, the top $x y$ plane $(z=10 \mathrm{~mm})$ was the furthest plane from the surface of the skull and had the highest values of the normalized cross-correlation for the majority of the grid points on this plane, Figure 3.3 (c). The normalized cross-correlation values were also distributed more uniformly on the $\boldsymbol{x y}$ plane furthest from the skull, Figure 3.3 (c). 
Another means of enhancing the PA images is by utilizing the imaging operator that is captured in the presence of the skull phantom. This imaging operator takes into account all the effects of the skull phantom on the propagation of the PA signal in the imaging volume. With this approach, the image resolution stays the same as the image that is captured in the absence of the skull. However, image reconstruction with this approach does not result in complete correction of the effects of the skull layer. For example, the length of the rod in the image that was reconstructed with the skull imaging operator was observed to be $0.6 \mathrm{~mm}$ smaller than the image that was reconstructed with the original imaging operator.

In reality, it is not possible to capture the imaging operator through the skull in live neonates, since we do not have physical access within the cavity of the skull of a neonate patient. However, it is possible to construct different skull phantoms with different thicknesses and different shapes, and choose the one that is the most similar to the shape of the neonate head. Then, the captured imaging operator through that skull phantom can be used to reconstruct the image of the neonate brain. Since the reconstruction time is less than a second, we can also reconstruct the data space of the neonate brain with several imaging operators, each captured through one skull phantom. Thus, we will have several PA images of the brain and we may choose the best one: for example, the PA image that has lowest noise when compared to the other images.

\subsection{Conclusion}

In this study, the effects of the skull on PA signal propagation were examined. The skull phantom distorted the PA signal in the form of signal attenuation and phase shifts. A skull-correction method was developed that utilized a corrected imaging operator to 
account for the attenuation and phase-shift distortions in the PA signals caused by the skull. The method was tested on captured data of a light-absorbing rod through the skull phantom. Images captured with the skull-corrected imaging operator have greater SNR than the images reconstructed with the original imaging operator captured in the absence of the skull. 


\section{References}

1. J. J. Volpe, "Neurobiology of periventricular leukomalacia in the premature infant," Pediatr. Res. 50, 553-562 (2001).

2. J. J. Volpe, "Cerebral white matter injury of the premature infant-more common than you think," Pediatrics 112, 176-180 (2003).

3. J. J. Volpe, "Encephalopathy of prematurity includes neuronal abnormalities," Pediatrics 116, 221-225 (2005).

4. B. Y. Huang and M. Castillo, "Hypoxic-ischemic brain injury: imaging findings from birth to adulthood," Radiographics 28, 417-39; quiz 617 (2008).

5. P. Tavakolian, R. Todd, I. Kosik, A. Chamson-Reig, F. Vasefi, K. S. Lawrence, and J. J. L. Carson, "Development of a neonatal skull phantom for photoacoustic imaging," Proc. SPIE. 8581, Photons Plus Ultrasound 8581, 858146 (2013).

6. P. Tavakolian, I. Kosik, A. R. Chamson, K. S. Lawrence, and J. J. L. Carson, "Potential for photoacoustic imaging of the neonatal brain," Proc. SPIE, Photons Plus Ultrasound 8581, 858147 (2013).

7. Y. Zhou, "High intensity focused ultrasound in clinical tumor ablation," World journal of clinical oncology 2, 8-27 (2011).

8. C. Huang, L. Nie, R. W. Schoonover, Z. Guo, C. O. Schirra, M. A. Anastasio, and L. V. Wang, "Aberration correction for transcranial photoacoustic tomography of primates employing adjunct image data," J. Biomed. Opt. 17, 066016 (2012).

9. H. Kirpalani and C. Nahmias, "Radiation risk to children from computed tomography," Pediatrics 121, 449-450 (2008).

10. J. White, G. T. Clement, and K. Hynynen, "Transcranial ultrasound focus reconstruction with phase and amplitude correction," IEEE Trans. Ultrason. Ferroelectr. Freq. Control 52, 1518-1522 (2005). 
11. R. W. Schoonover and M. A. Anastasio, "Compensation of shear waves in photoacoustic tomography with layered acoustic media," J. Opt. Soc. Am. A Opt. Image Sci. Vis. 28, 2091-2099 (2011).

12. R. W. Schoonover, L. V. Wang, and M. A. Anastasio, "Numerical investigation of the effects of shear waves in transcranial photoacoustic tomography with a planar geometry," J. Biomed. Opt. 17, 061215 (2012). 


\section{Chapter 4}

\section{Summary and future work}

\subsection{Overview}

This chapter concludes the dissertation and summarizes the results that have been described in chapters 2 and 3. Additionally, it provides a perspective on our research and a view to future work.

\subsection{Skull phantom fabrication}

An acoustically realistic neonatal skull phantom was constructed to assess PAI through the skull of premature infants. The fabrication study was composed of two parts: first, the acoustic insertion loss of a variety of candidate materials was measured and a material that closely mimicked the ultrasonic properties of the neonatal skull bone was selected; second, a skull phantom was fabricated utilizing the selected material, using a mold of a neonatal skull.

\subsubsection{Skull mimicking material}

Frequency-dependent ultrasonic insertion loss was measured for a variety of materials, including polyvinyl chloride, acrylic ( 3 and $12.5 \mathrm{~mm}$ thick), plastic (12.5 $\mathrm{mm}$ thick), polycarbonates (12.6 mm thick), and cured mixtures of epoxy and titanium powder (different thicknesses). Measurements revealed large differences between the acoustic properties of the skull bone (as reported in ref [1,2] ) and materials such as polycarbonate sheet, acrylic, plastic, and polyvinyl chloride. Samples prepared from mixtures of epoxy and graphite powder were also substantially different from neonatal skull bone. The acoustic properties of epoxy samples mixed with titanium dioxide powder were the 
closest to that of neonatal skull bone. To fine-tune the properties of the epoxy-titanium dioxide powder mixtures, samples were prepared with different proportions of epoxy and titanium dioxide powder.

Based on our measurements, the acoustic insertion loss increased when either the thickness of the samples, or the proportion of titanium dioxide powder, was increased. The acoustic insertion loss of a 1.4-mm-thick sample composed of 50\% titanium dioxide powder and 50\% epoxy was the closest match to the acoustic insertion loss of the neonatal skull bone across a frequency range of 0.9 to $3 \mathrm{MHz}$.

\subsubsection{Skull mold fabrication}

We utilized 3D prototyping techniques to scan the head of a realistic infant doll to design a mold and fabricated it from Teflon ${ }^{\circledR}$ using a $\mathrm{CNC}$ machine. The gap between the top and bottom pieces of the mold was filled with a mixture of epoxy and titanium dioxide powder. After two hours of curing at room temperature, the mixture was removed from the mold and exposed to air. Skull phantoms with different transcranial thicknesses were constructed utilizing molds with various gaps between the two halves. In one example, the skull phantom had a transcranial thickness of $1.52 \mathrm{~mm}$, a length of $79.3 \mathrm{~mm}$, a width of $63 \mathrm{~mm}$, and a height of $31.5 \mathrm{~mm}$.

\subsection{Effects of the skull layer on photoacoustic signals}

Two photoacoustic signals, one captured through the skull and the other in the absence of the skull, were compared to determine the effect of the skull layer on the photoacoustic signal propagation in terms of insertion loss and time delay. 
The insertion loss caused by the skull phantom was measured to be approximately 4.45 $\mathrm{dB}$ by the transducer $(2.7 \mathrm{MHz}$ central frequency and $127 \%$ bandwidth), almost perpendicular to the skull and the region of interest. The insertion loss was estimated from the ratio of the peak-to-peak amplitude between the two photoacoustic signals. The result was close to the acoustic insertion loss of the skull bone, $4 \mathrm{~dB}$ at $1 \mathrm{MHz}$ and increased mildly with frequency.

We expected a $0.37 \mu$ s difference in time of arrival of the signal based on the difference between the speed of sound in water $(1.5 \mathrm{~mm} / \mu \mathrm{s})$ and the speed of sound in a cured mixture of epoxy and titanium dioxide powder $(2.35 \mathrm{~mm} / \mu \mathrm{s})$. Measured experimentally in the presence of the $1.52 \mathrm{~mm}$ thick skull, the photoacoustic signal arrived around 0.32 $\mu$ s sooner than in the absence of the skull. This was close to the expected value.

Experimental estimation of the imaging operator with and without the skull phantom provided a method for analyzing the effect of an intervening layer on photoacoustic signal propagation, with a fine level of detail (i.e. $0.5 \mathrm{~mm}$ grid spacing). The first analysis involved the construction of a delay matrix by measuring the difference between the arrival time for the photoacoustic signal with and without the skull. The time delay was estimated from the temporal shift required to maximize the cross-correlation between the two time-series from the same transducer and the same grid location. The majority of time delay estimates ranged between 0.1 and $0.5 \mu$ s based on an effective sampling rate of $40 \mathrm{MHz}$. The ratio of the peak photoacoustic signals with and without the skull was measured at each transducer for the photoacoustic point source positioned at each grid location. Most of the ratios were ranged between 0.3 to 0.6 , which was expected, based 
on the acoustic properties of the $1.4 \mathrm{~mm}$-thick mixture of 50\% epoxy and 50\% titanium dioxide.

In chapter 2, subsets of the imaging operators with and without the skull layer and in different areas of the imaging volume were presented and compared. In the presence of the skull phantom, the photoacoustic signal patterns became weaker, were broken in places, were sometimes followed closely by a weak noisy pattern, and were shifted in time. The weakness of the photoacoustic signal, and the broken areas, resulted either from amplitude attenuation caused by the skull, or acoustic reflection at the surface of the skull. This acoustic reflection was sometimes detected by a transducer and formed a noisy pattern.

The non-uniform thickness $(1.52 \pm 0.12 \mathrm{~mm})$ of the skull phantom, i.e. thinner at the center, caused different amplitude attenuation and varied time of arrival of the acoustic signals to the transducers. Therefore, the distortion of the photoacoustic signals that are generated around the center of the transducer array was lower than in other areas of the transducer array.

Another way of studying the effects of the skull on different locations of the imaging volume is through utilizing the cross-correlation operator, which was explained in chapter 3. The normalized cross-correlation of two signals is a measure of the similarity of the signals.

Accordingly, we computed the cross-correlation of the PA signals captured through the skull and in the absence of the skull at all transducers for each of the grid locations of the imaging operator. The cross-correlation displayed the extent of the difference between 
the PA signals captured in the presence and the absence of the skull at all grid points of the imaging volume. At the grid points that were located close to the surface of the skull phantom, the normalized cross-correlation values were very low, distributed from 0 to 0.2. This implies that the PA signals propagated through the skull experience significant distortion. However, the normalized cross-correlation values were high for the grid points that were far from the surface of the skull. This fact demonstrated that the skull distortion of the PA signals was greater for the grid points that were closer to the skull surface.

\subsection{Imaging results}

Photoacoustic images of vessel-like phantoms filled with various concentrations of ICG $(500,250,100,50$, and $1.25 \mu \mathrm{M})$ in the presence and absence of the skull phantom were captured. The PAI system utilized was capable of imaging various concentrations of ICG through the skull phantom. The lowest ICG concentration detectable through the skull phantom was $50 \mu \mathrm{M}, 40$-fold higher than that detectable by PAI with no skull phantom; this was due to the $4.45 \mathrm{~dB}$ attenuation of the acoustic signal from the skull phantom. The attenuated photoacoustic signals were thus not reconstructable for concentrations lower than $50-\mu \mathrm{M}$ ICG. The contrast of the images decreased when the ICG concentration decreased. The photoacoustic images collected in the absence of the skull phantom were observed to have higher contrast than the images collected in the presence of the skull phantom.

\subsection{Skull correction method}

Two skull-correction methods were developed in order to enhance the images of the objects through the skull. The first method corrected the original imaging operator 
(captured in the presence of the skull) in terms of amplitude attenuation and phase shift. This method could be a basis for developing a mathematical model of the effects of the skull, and thus correcting the PA signals propagated through the skull. The second method used the imaging operator captured through the skull. This method can correct PA signals from all of the effects of the skull; however, it can only be used to correct the images of objects through the skull phantom, and not an infant skull.

These methods were tested by performing another PA imaging experiment. An image of an optically-absorbing rod in the presence and absence of the skull phantom were captured. Then, the data space of the rod captured through the skull was reconstructed with both the skull-corrected imaging operator and the imaging operator captured in the presence of the skull. The image reconstructed with the skull-corrected imaging operator had lower noise compared to the image reconstructed with the original imaging operator. The data space of the rod was then reconstructed with the imaging operator captured through the skull, which resulted in the clearest image of the rod captured through the skull.

\subsection{Conclusion}

A mixture of epoxy and titanium dioxide powder was selected as a skull-mimicking material. A skull-mimicking phantom with an average thickness of $1.52 \mathrm{~mm}$ made of $56 \%$ epoxy and $44 \%$ titanium dioxide was fabricated. The skull phantom was used to study the effects of a neonatal skull on PA signal propagation. The skull phantom distorted the PA signal in the form of signal attenuation and phase shift. Calibration scans of the system with and without the skull were acquired and compared, and were used for image reconstruction. Photoacoustic images of vessel-like tubes filled with ICG were 
captured. The photoacoustic imaging system was able to capture images of tubes

containing ICG at a concentration of $1.25 \mu \mathrm{M}$. In the presence of the skull, however, the system detected the ICG only when the concentration was greater than $50 \mu \mathrm{M}$. An image correction method was developed and tested on images of an optically-absorbing rod. The skull-correction method demonstrated that the effects of the skull on photoacoustic signals are measurable and correctable.

\subsection{Future work}

A potential clinical application of PAI is detection of periventricular leukomalacia in premature infants. Periventricular leukomalacia occurs when the tissue around the ventricles in the brain is damaged. A potential next step of this project could be the acquisition of images of more anatomically correct phantoms. Therefore, the ventricles and white matter could be molded into the skull phantom, and simulated lesions could be embedded nearby the ventricle in the white matter. Then, the PAI system could be challenged to capture PA images of simulated PVL through the skull and the brain phantom.

As mentioned earlier, capturing the imaging operator with an actual neonatal skull is not possible. However, it is possible to fabricate many phantoms with different characteristics such as different shapes or different thicknesses. Thus, based on the characteristics of a specific infant skull (e.g. skull thickness), one phantom that simulates the skull of a specific neonatal patient could be selected. By using the imaging operator captured in the presence of a closely matched phantom, an image of the neonate's brain could be reconstructed. This would correct the brain image from the effects of the skull to a high degree, especially for regions far from the skull. By preparing the preliminary 
phantom data such as the imaging operator, the image correction could be rapid, possibly taking less than a minute. However, the proposed method needs to answer a critical question: Should the position of the neonatal skull corresponding to the imaging cavity be exactly the same as the position of the skull phantom corresponding to the imaging cavity? To answer this question, an imaging experiment was performed with the same imaging system, image reconstruction algorithm, and calibration scan explained in chapter 3. In the experiment, we investigated the effect of rotating the skull phantom in the transducer array on imaging the optically-absorbing rod. The skull phantom was placed in the imaging cavity at two different angles $\left(0^{\circ}\right.$, and $\left.37^{\circ}\right)$, and images of the rod were captured through the skull at both angles.

Error! Reference source not found.Figure 3.3 (a-b) shows the 3D CAD model of the skull in the transducer array at $0^{\circ}$ and $37^{\circ}$ angles. Images of the rod reconstructed with the original imaging operator at $0^{\circ}$ and $37^{\circ}$ are shown in Error! Reference source not found.Figures 3.3(c) and (d). 


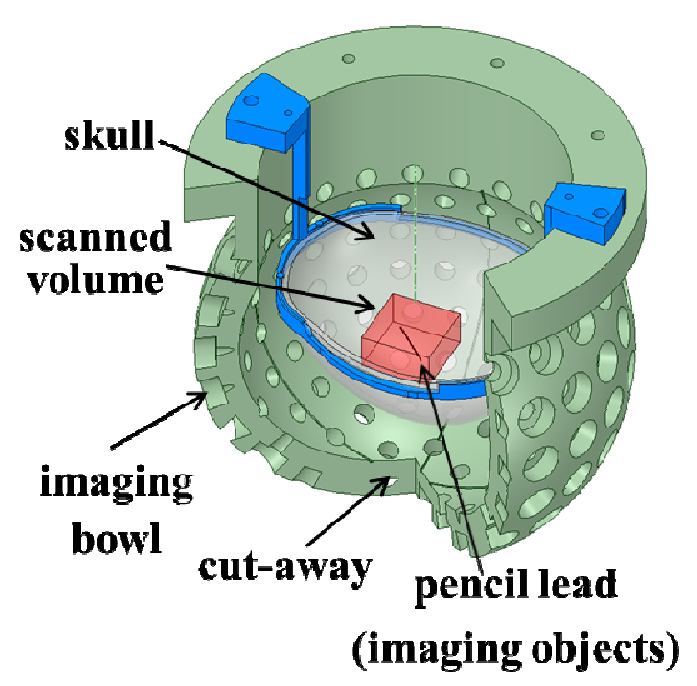

(c)

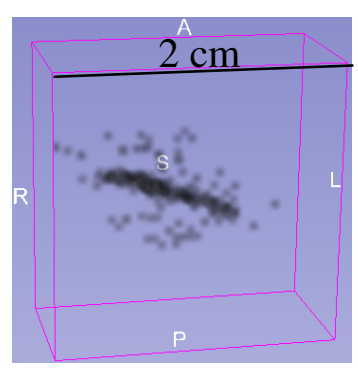

(d)

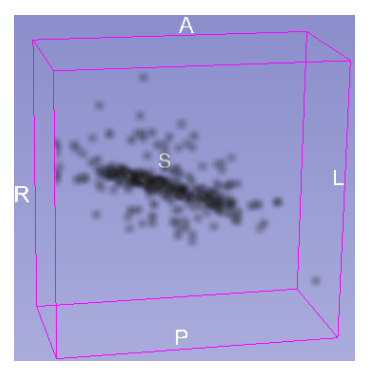

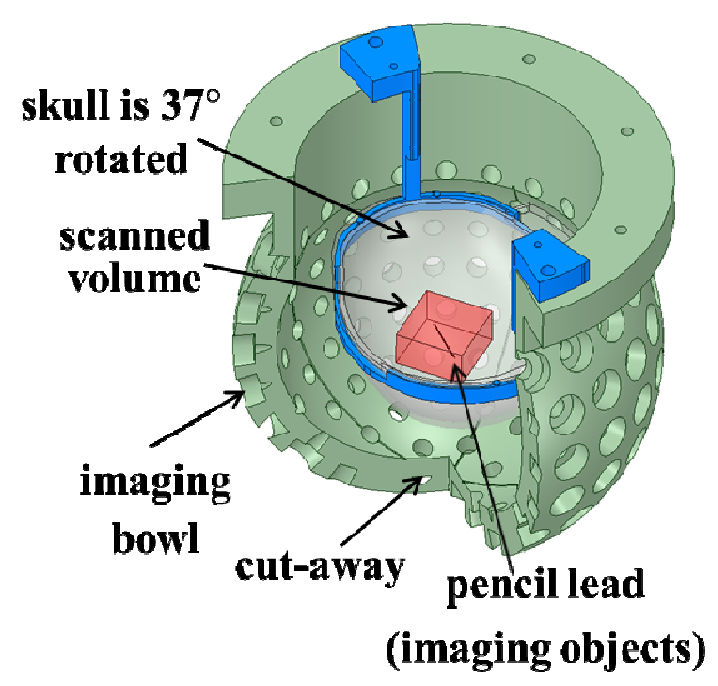

(e)
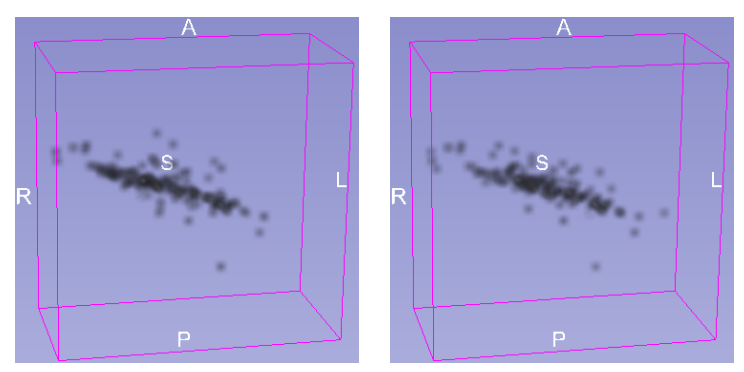

Figure 4.1. (a) A 3D CAD model of the skull position in relation to the transducer array, the imaging volume, and an optically-absorbing rod. (b) A 3D CAD model of the skull position, which was turned $37^{\circ}$ in the xy plane. (c) Image of the rod through the skull reconstructed with the original imaging operator. The skull was located in the same place as that where the imaging operator was captured (shown in (a)). (d) Image of the rod through the skull that was turned $37^{\circ}$ in the xy plane. The image is reconstructed with the original imaging operator. (e) An image of the rod reconstructed with the imaging operator that was captured through the skull. Capturing the signal of the rod, and the imaging operator, were both at $0^{\circ}$ (f) Image 
of the rod reconstructed with the imaging operator that was captured through the skull. To capture the data of the rod the skull was rotated $37^{\circ}$, but to capture the imaging operator the skull was at $0^{\circ}$.

Error! Reference source not found.Figure 3.3 (a-b) shows the 3D CAD model of the skull in the transducer array at $0^{\circ}$ and $37^{\circ}$ angles. Images of the rod reconstructed with the original imaging operator at $0^{\circ}$ and $37^{\circ}$ are shown in Error! Reference source not found.Figures 3.3(c) and (d).

The experiment demonstrated that the location of the skull phantom in relation to the imaging cavity did not have a large effect on the image of the object. Therefore, the position of the neonatal skull in relation to the imaging cavity may not need to be exactly the same as the position of the skull phantom corresponding to the transducer array. 


\section{References}

1. X. Wang, J. B. Fowlkes, D. L. Chamberland, G. Xi, and P. L. Carson, "Reflection mode photoacoustic imaging through infant skull toward noninvasive imaging of neonatal brains," Proc. SPIE, Photons Plus Ultrasound 7177, 717709 (2009).

2. X. Wang, D. L. Chamberland, and G. Xi, "Noninvasive reflection mode photoacoustic imaging through infant skull toward imaging of neonatal brains," J. Neurosci. Methods 168, 412-421 (2008). 


\section{Curriculum Vitae}

Name:

Post-secondary Education and Degrees:

Honours and Awards:

Related Work Experience
Pantea Tavakolian

Shariaty Technical University

Tehran, Iran 2004-2008.

The University of Western Ontario (UWO)

London, Ontario, Canada

2011-2014 M.A.

SPIE travel Scholarship $(\$ 2,000)$

2013

Western Graduate Research Scholarship, UWO, London, Ontario $(\$ 21,600)$

2011-2013

Scholarship for top B.Sc student in the Department of Electrical engineering, Shariaty Technical University

2006

Research Assistant

The University of Western Ontario

2011-2014

Teaching Assistantship

Shariaty University

2007-2008

\section{Publications:}

$\underline{\text { Under Revision }}$

Pantea Tavakolian, Kosik, I, Keith St. Lawrence, and Carson, JJL. Investigation of 3D photoacoustic neonatal brain imaging using a skull-mimicking phantom. Journal of Biomedical Optics, In Preparation.

Pantea Tavakolian, Kosik, I, Keith St. Lawrence, and Carson, JJL. 3D photoacoustic imaging through the skull phantom with phase and amplitude correction. Journal of Biomedical Optics, In Preparation. 


\section{Proceeding publications, Abstracts and Conference Presentations:}

1. P.Tavakolian, Jeffrey J.L. Carson, "Effects of the skull on photoacoustic signals and images", Podium presentation at Artimino Ultrasound Conference, Lake Rosseau, Ontario (2013).

2. Pantea Tavakolian, Ivan Kosik, Astrid Chamson-Reig, Keith St. Lawrence, and Jeffrey J.L. Carson, "Potential for photoacoustic imaging of the neonatal brain", SPIE Photons Plus Ultrasound, (2013).

3. Pantea Tavakolian, Rhiannon Todd, Fartash Vasefi, Ivan Kosik, Astrid Chamson-Reig, Keith St. Lawrence, and Jeffrey J.L. Carson "Developement of a neonatal skull mimicking phantom for photoacoustic imaging of the infant brain", SPIE Photons Plus Ultrasound, (2013).

4. Pantea Tavakolian, Ivan Kosik, Keith St. Lawrence, and Jeffrey J.L. Carson Investigation of 3D photoacoustic neonatal brain imaging using a skullmimicking phantom. Poster presentation at 2013 London Health Research Day.

5. Pantea Tavakolian, Ivan Kosik, Keith St. Lawrence, and Jeffrey J.L. Carson Investigation of photoacoustic imaging of the neonatal brain. Poster presentation at 2012 London Health Research Day.

6. Pantea Tavakolian, Ivan Kosik, Keith St. Lawrence, and Jeffrey J.L. Carson Fabrication of neonatal skull phantom for photoacoustic imaging. Poster presentation at 2011 London Health Research Day.

7. P. Tavakolian, H. Gharaee, "A New Low-Complexity and Low-Power BCSE Method for FIR Filter Implementation", IEEE regional symposium on micro and nano electronics, p. 364-368 (2009).

8. Hossein Gharaee, Pantea Tavakolian, Abdolreza Nabavi "Low Complexity and Low Power of OTR-UWB Baseband", Iranian Conference on Electrical Engineering ICEE2009_2548 (2009).

9. P.Tavakolian, H. Gharaee "A New 5-bit BCSE Method for Implementing Low Complexity and Low Power Reconfigurable FIR Filters", Iranian Conference on Electrical Engineering ICEE2009_2640 (2009). 